\title{
Review
}

\section{A revision of the descriptions of ectomycorrhizas published since 1961}

\author{
Miriam DE ROMAN ${ }^{1,3}$, Vanessa CLAVERIA ${ }^{2}$ and Ana Maria DE MIGUEL ${ }^{2}$ \\ ${ }^{1}$ CABI Bioscience, Bakeham Lane, Egham, Surrey TW20 9TY, UK. \\ ${ }^{2}$ Department of Botany, University of Navarra, ES-31080 Pamplona, Spain. \\ ${ }^{3}$ Department of Agriculture \& Forestry, School of Biological Sciences, University of Aberdeen, Hilton Campus, \\ Block M, Aberdeen AB24 4FA, UK. \\ E-mail:miriamderoman@hotmail.com
}

Received 5 March 2005; accepted 17 June 2005.

\begin{abstract}
All available publications providing descriptions of ectomycorrhizas (ECM) were reviewed in order to build a database containing details on fungus forming the ECM, host tree, country where the material for description was collected, and habitat of the ECM. Other secondary data were also recorded. In all 1244 descriptions of ECM published since 1961 in 479 papers were reviewed. The number of different ECM morphotypes described was 814. Most ECM described were collected in Europe and North America. Gymnosperms were the most common tree associates, and boreal and temperate forests the most studied ecosystems. Fungal symbionts were mostly Basidiomycota, epigeous, and with mushroom-like morphology. The paper also addresses the gaps in ECM knowledge that mycorrhizologists should address in future studies.
\end{abstract}

\section{INTRODUCTION}

Research on ectomycorrhizas (ECM) has evolved greatly over the last 40 years. From the first morphological analyses to the latest ecological, physiological and genetic studies, the range of information now available is outstanding. Despite all the scientific advances, the identification of the fungi involved remains one of the first steps to be followed when studying ECM communities. Molecular techniques are efficient tools to achieve this aim, but the morphological and anatomical features of the ECM are still an invaluable source of information for the better understanding of the fungal component.

Many ECM morphotypes have been more or less accurately described since the beginning of ectomycorrhizal symbiosis research in the late 19th century. A few authors have tried to establish classification systems in order to develop identification keys paralleling those available for plants and animals, but this is a difficult task. Dominik (1969) made a first attempt. A few years later Zak (1973) stated that a detailed description of each ECM was essential for its identification, and that this description should be based on several collections in order to note possible variations in the ECM over different developmental stages.
In spite of these general ideas, the methodology used by each author when describing an ECM varied greatly in descriptions published before 1986, when Agerer (1986a, 1987-2002, 1994a, 1999a) began to publish guidelines for the systematic of description and identification of ECM widely used nowadays. In addition, Agerer created a binomial nomenclature system for those ECM described but not yet identified, edited a Colour Atlas of Ectomycorrhizas (Agerer 1987-2002) with photographs of the ECM described to facilitate identification by comparison, and developed computer software to characterize and determine ECM (Agerer \& Rambold 1998).

Other approaches for the description and identification of ECM also exist. Ingleby et al. (1990) developed a system to name the features of ECM, and published 24 brief descriptions of ECM including photographs and drawings.

Another widely used methodology is the so-called Concise Descriptions of North American Ectomycorrhizas, developed by Goodman et al. (1996-2000). The descriptions published according to this system are more detailed than those in Ingleby et al. (1990), but they lack the level of detail of the system proposed by Agerer (1986a, 1987-2002, 1994a, 1999a), and are confined to the North American geographical region. 
Table 1. Compilation of the 1244 ECM descriptions reviewed. ECM descriptions are ordered alphabetically by name of ECM and then by reference, with details of the host tree, the country where the material was collected, and the habitat where the ECM was found (1, field; 2, synthesized in vitro; 3 , nursery; 4, truffle ground stand; 5 , various).

\begin{tabular}{|c|c|c|c|c|}
\hline Ectomycorrhiza & Reference & Host tree & Country & Habitat \\
\hline Abierhiza fascicularis & Haug et al. (1994) & Abies kawakamii & Taiwan & 1 \\
\hline A. tomentosa & Haug et al. (1994) & A. kawakamii & Taiwan & 1 \\
\hline \multirow[t]{8}{*}{ AD (angle droit, Giraud 1988) } & Águeda, Fernández \& De Miguel (2001) & Quercus ilex & Spain & 4 \\
\hline & Bencivenga et al. (1995) & Not specified & Italy & 3 \\
\hline & De Román \& De Miguel (2005) & Q. ilex & Spain & 1 \\
\hline & Di Massimo et al. (1996) & Q. ilex & Spain & 3 \\
\hline & Etayo \& De Miguel (1998) & Q. faginea, $Q$. ilex & Spain & 4 \\
\hline & Giraud (1988) & Not specified & France & 4 \\
\hline & Granetti \& Baciarelli (1997) & Q. ilex & Italy & 4 \\
\hline & Sáez \& De Miguel (1995) & Not specified & Spain & 4 \\
\hline \multirow[t]{2}{*}{ Agaricales } & Buée, Vairelles \& Garbaye (2004) & Fagus sylvatica & France & 1 \\
\hline & Kaldorf (2004) & Populus tremula $\times P$. tremuloides & Germany & 1 \\
\hline \multirow{3}{*}{ Albatrellus ovinus } & Agerer (1996b) & Picea abies & Germany & 1 \\
\hline & Agerer (1996g) & P. abies & Germany & 1 \\
\hline & Agerer et al. (1996a) & P. abies & Germany & 1 \\
\hline Alnirhiza atroverrucosa & Pritsch, Munch \& Buscot (1997) & Alnus glutinosa & Germany & 1 \\
\hline A. cremicolor & Pritsch et al. (1997) & A. glutinosa & Germany & 1 \\
\hline A. cystidiobrunnea & Pritsch et al. (1997) & A. glutinosa & Germany & 1 \\
\hline A. texta & Pritsch et al. (1997) & A. glutinosa & Germany & 1 \\
\hline \multirow{3}{*}{ Alpova diplophloeus } & Godbout \& Fortin (1983) & A. crispa, A. rugosa & Canada & 2 \\
\hline & Miller, Koo \& Molina (1990) & A. rubra & USA & 5 \\
\hline & Wiedmer, Senn-Irlet \& Agerer (2001) & A. viridis & Switzerland & 1 \\
\hline Amanita & Godbout \& Fortin (1985) & Populus tremuloides & Canada & 3 \\
\hline A. caesarea & Meotto et al. (1997) & Quercus & Italy & 1 \\
\hline \multirow[t]{2}{*}{ A. citrina } & Mleczko (2002a) & Pinus sylvestris & Poland & 1 \\
\hline & Mleczko (2004a) & P. sylvestris & Poland & 1 \\
\hline \multirow[t]{2}{*}{ A. muscaria } & Chu-Chou \& Grace (1983) & Pseudotsuga menziesii & N. Zealand & 1 \\
\hline & Ingleby et al. (1990) & Betula pendula & UK & 1 \\
\hline A. spissa & Torres \& Honrubia (1994) & Pinus halepensis & Spain & 2 \\
\hline \multirow{3}{*}{ Amphinema } & Hagerman et al. (1999) & Not specified & Canada & 1 \\
\hline & Hagerman, Sakakibara \& Durall (2001) & Pseudotsuga menziesii & Canada & 1 \\
\hline & Jones et al. (1997) & Betula papyrifera, $P$. menziesii & Canada & 5 \\
\hline \multirow[t]{10}{*}{ A. byssoides } & Danielson \& Pruden (1989) & Picea pungens & Canada & 1 \\
\hline & Danielson, Zak \& Parkinson (1984) & Pinus banksiana & Canada & 3 \\
\hline & Fassi \& De Vecchi (1962) & P. strobus & Italy & 3 \\
\hline & Haug et al. (1994) & P. taiwanensis & Taiwan & 1 \\
\hline & Ingleby et al. (1990) & Picea sitchensis & UK & 1 \\
\hline & Kernaghan (2001) & $\begin{array}{l}\text { Abies lasiocarpa, Dryas octopetala, } \\
\text { Picea engelmanii, Salix barrattiana }\end{array}$ & Canada & 1 \\
\hline & Massicotte et al. (1999b) & $\begin{array}{l}\text { A. grandis, Arbutus menziesii, Lithocarpus } \\
\text { densiflora, Pinus ponderosa, } \\
\text { Pseudotsuga menziesii }\end{array}$ & USA & 3 \\
\hline & Montecchio et al. (2002) & Quercus ilex & Italy & 1 \\
\hline & Weiss (1989) & Picea abies & Germany & 1 \\
\hline & Weiss (1991a) & P. abies & Germany & 2 \\
\hline
\end{tabular}

Weiss (1989)

P. abies
Germany 
A. byssoides-like

Arcangeliella asterosperma

A. borziana

Ascomycota

Astraeus hygrometricus

Austropaxillus boletinoides

Balsamia alba

Bankera fuligineo-alba

Basidiomycota

Betulaerhiza hystrix

Boletaceae

Boletinus cavipes

Boletopsis leucomelaena

Boletus

B. aereus
Harniman \& Durall (1996a)

Luppi \& Gautero (1967)

Egli et al. (2001)

Al Sayegh \& Kraigher (2000)

Haug et al. (2005)

Ingleby et al. (1990)

Ingleby et al. (1990)

Kernaghan (2001)

Kernaghan (2001)

Danielson (1984b)

Giraud (1988)

Schramm (1966)

Palfner (2001)

Palfner (2001)

Palfner (2002a)

Palfner \& Agerer (1998a)

Palfner (1998a)

Agerer \& Otto (1997)

Agerer \& Otto (1998)

Danielson (1984b)

Jones et al. (1997)

Jones et al. (1997)

Jones et al. (1997)

Jones et al. (1997)

Jones et al. (1997)

Jones et al. (1997)

Jones et al. (1997)

Jones et al. (1997)

Jones et al. (1997)

Jones et al. (1997)

Massicotte et al. (1999b)

Massicotte et al. (1999b)

Massicotte et al. (1999b)

Massicotte et al. (1999b)

Müller \& Agerer (1990)

Müller (1994)

De Román \& De Miguel (2005)

Kaldorf (2004)

See, Alexander \& Watling (1997)

Selosse, Bauer \& Moyersoen (2002)

Treu (1990a)

Treu (1990b)

Agerer (1992a)

Agerer (1993a)

Chu-Chou \& Grace (1983)

Schramm (1966)

Ceruti et al. (1985)
P. engelmannii

Quercus pubescens

Picea abies

Fagus

Betula pendula

Picea sitchensis

Dryas octopetala

Salix barrattiana

Pinus banksiana

Not specified

Pinus virginiana

Nothofagus betuloides

$N$. pumilio

N. pumilio

Pinus jeffreyi

P. jeffreyi

P. sylvestris

P. sylvestris
P. banksiana

Betula papyrifera, Pseudotsuga menziesi

$B$. papyrifera, $P$. menziesil

$B$. papyrifera, $P$. menziesii

B. papyrifera, $P$. menziesii

$B$. papyrifera, $P$. menziesii

B. papyrifera, P. menziesii

B. papyrifera, $P$. menziesii

B. papyrifera, $P$. menziesii

Pseudotsuga menziesii

Pseudotsuga menziesi

Abies grandis, Lithocarpus densiflora,

Pinus ponderosa, Pseudotsuga menziesi

Abies grandis, P. menziesii

Arbutus menziesii, P. menziesii

Pinus ponderosa

Betula pendula

B. pendula

Quercus ilex

Populus tremula $\times$ P. tremuloide

Shorea leprosula

Carpinus

Larix decidua

L. decidua

Picea abies

P. abies

Pseudotsuga menziesii

Pinus virginiana

Castanea sativa 
Table 1. (Cont.)

\begin{tabular}{|c|c|c|c|c|}
\hline Ectomycorrhiza & Reference & Host tree & Country & Habitat \\
\hline Boletus badius & Voiry (1981) & Quercus & France & 1 \\
\hline \multirow[t]{6}{*}{ B. edulis } & Agerer \& Gronbach (1990a) & Picea abies & Germany & 1 \\
\hline & Ceruti et al. (1987) & P. excelsa & Italy & 2 \\
\hline & Ceruti et al. (1987) & Pinus sylvestris & Italy & 2 \\
\hline & Gronbach (1988) & Picea abies & Germany & 1 \\
\hline & Luppi \& Gautero (1967) & Quercus robur & Italy & 1 \\
\hline & Meotto \& Pellegrino (1989) & $\begin{array}{l}\text { Castanea crenata, C. sativa, Quercus cerris, } \\
\text { Quercus rubra }\end{array}$ & Italy & 3 \\
\hline B. luridus & Brunner et al. (1992) & Picea abies & Switzerland & 2 \\
\hline B. loyo & Palfner (2001) & Nothofagus oblicua & Chile & 1 \\
\hline B. pinophilus & Duñabeitia et al. (1996) & Pinus radiata & Spain & 3 \\
\hline B. putidus & Palfner (2001) & Nothofagus oblicua & Chile & 1 \\
\hline B. rhodoxanthus & Hahn (2001) & Cistus ladanifer & Germany & 1 \\
\hline \multirow[t]{4}{*}{ Byssocorticium atrovirens } & Brand (1991f) & Fagus sylvatica & Germany & 1 \\
\hline & Brand (19911) & F. sylvatica & Germany & 1 \\
\hline & Buée et al. (2004) & F. sylvatica & France & 1 \\
\hline & Luppi \& Gautero (1967) & Quercus petraea & Italy & 1 \\
\hline \multirow[t]{2}{*}{ Byssoporia terrestris } & Massicotte et al. (1999b) & $\begin{array}{l}\text { Abies grandis, Arbutus menziesii, } \\
\text { Lithocarpus densiflora, Pinus ponderosa, } \\
\text { Pseudotsuga menziesii }\end{array}$ & USA & 3 \\
\hline & Zak \& Larsen (1978) & P. menziesii & USA & 1 \\
\hline \multirow[t]{2}{*}{ Cantharellus cibarius } & Mleczko (2002b) & Pinus sylvestris & Poland & 1 \\
\hline & Mleczko (2004b) & P. sylvestris & Poland & 1 \\
\hline C. formosus & Countess \& Goodman (2000) & Tsuga heterophylla & Canada & 1 \\
\hline Castanopsirhiza glabra & Haug et al. (1994) & Castanopsis borneensis & Taiwan & 1 \\
\hline \multirow[t]{19}{*}{ Cenococcum geophilum } & Abourouh \& Najim (1995) & Cedrus atlantica & Morocco & 1 \\
\hline & Agerer \& Gronbach (1988) & Picea abies & Germany & 1 \\
\hline & Águeda et al. (2001) & Quercus ilex & Spain & 4 \\
\hline & Al Sayegh \& Kraigher (1999) & Picea abies & Slovenia & 1 \\
\hline & Azul \& Freitas (1999) & Quercus suber & Portugal & 1 \\
\hline & Baxter et al. (1999) & Ouercus & USA & 1 \\
\hline & Bradbury (1998) & Pinus contorta & Canada & 1 \\
\hline & Bradbury, Danielson \& Visser (1998) & P. contorta & Canada & 1 \\
\hline & Buée et al. (2004) & Fagus sylvatica & France & 1 \\
\hline & Danielson \& Pruden (1989) & Picea pungens & Canada & 1 \\
\hline & De Román \& De Miguel (2005) & Ouercus ilex & Spain & 1 \\
\hline & Di Battista et al. (2002) & Pseudotsuga menziesii & USA & 3 \\
\hline & Di Massimo et al. (1996) & Pinus nigra & Spain & 3 \\
\hline & Dickie et al. (2004) & Helianthemum bicknellii & USA & 1 \\
\hline & Donnini \& Bencivenga (1995) & Corvlus avellana, Ouercus pubescens & Italy & 4 \\
\hline & Fontana \& Centrella (1967) & Quercus petraea & Italy & 1 \\
\hline & Giraud (1988) & Not specified & France & 4 \\
\hline & Godbout \& Fortin (1983) & Alnus crispa, A. rugosa & Canada & 2 \\
\hline & Godbout \& Fortin (1985) & Populus tremuloides & Canada & 3 \\
\hline
\end{tabular}


Gronbach (1988)

Hagerman et al. (1999)

Hagerman et al. (2001)

Harniman \& Durall (1996b)

Ingleby et al. (1990)

Jany, Garbaye \& Martin (2002)

Jones et al. (1997)

Kaldorf (2004)

Kernaghan (2001)

Massicotte et al. (1999b)

Moser et al. (2005)

Palfner (2001)

Pigott (1982)

Rose, Gerald Van Dyke \& Davey (1981)

Schramm (1966)

See et al. (1997)

Torres \& Honrubia (1997)

Voiry (1981)

Wöllecke (2001)

Raidl (1999)

De Román \& De Miguel (2005)

Agerer (1990a)

Agerer (1990b)

Agerer (1990a)

Tedersoo et al. (2003)

Tedersoo et al. (2003)

Tedersoo et al. (2003)

Buée et al. (2004)

Danielson (1984b)

Selosse et al. (2002)

Agueda et al. (2001)

Bradbury (1998)

Bradbury et al. (1998)

Buée et al. (2004)

Buée et al. (2004)

Buée et al. (2004)

Dickie et al. (2004)

Godbout \& Fortin (1985)

Jakucs (2002a)

Kernaghan (2001)

Kernaghan (2001)

Treu (1990a)

Voiry (1981)

Pritsch et al. (1997)

Wiedmer \& Senn-Irlet (1999a)
Picea abies

Pseudotsuga menziesi

Picea engelmannii

P. sitchensis

Fagus sylvatica

Betula papyrifera, Pseudotsuga menziesii

Populus tremula $\times$ P. tremuloides

Abies lasiocarpa, Dryas octopetala,

Picea engelmanii, Salix barrattian

Abies grandis, Arbutus menziesii,

Lithocarpus densiflora, Pinus ponderosa,

Pseudotsuga menziesi

Quercus garryana

Nothofagus pumilio

Tilia cordata

Eucalyptus nova-anglica

Populus tremuloides

Shorea leprosula

Pinus halepensis

Fagus

Pinus sylvestris

Picea abies

Quercus ilex

Picea abies

P. abies

Pinus sylvestris

Betula pendula

Picea abies

Betula pendula

Fagus sylvatica

Pinus banksiana

Not specified

Quercus ilex

Pinus contorta

P. contorta

Fagus sylvatica

F. sylvatica

F. sylvatica

Helianthemum bicknellii

Populus tremuloides

P. alba

Picea engelmannii

Salix barrattiana

Larix decidua

Quercus

Alnus glutinosa

A. viridis
C. alneus

C. alnobetulae

Germany

Germany

Germany

Estonia

Estonia

Estonia

France

Canada

Canada

France

France

France

USA

Canada

Canada

Canada

Germany

France

Germany 
Table 1. (Cont.)

\begin{tabular}{|c|c|c|c|c|}
\hline Ectomycorrhiza & Reference & Host tree & Country & Habitat \\
\hline Cortinarius amoenolens & Hagerman et al. (1999) & Not specified & Canada & 1 \\
\hline C. armillatus & Cuvelier \& Agerer (1991) & Betula pendula & Belgium & 1 \\
\hline C. atropusillus & Wiedmer \& Senn-Irlet (1999b) & Alnus viridis & Switzerland & 1 \\
\hline \multirow[t]{2}{*}{ C. austrosalor } & Palfner (2001) & Nothofagus nitida & Chile & 1 \\
\hline & Palfner (2002b) & $N$. nitida & Chile & 1 \\
\hline C. badiovestitus & Wiedmer \& Senn-Irlet (1999c) & Alnus viridis & Switzerland & 1 \\
\hline \multirow[t]{2}{*}{ C. bibulus } & Miller et al. (1990) & A. rubra & USA & 5 \\
\hline & Wiedmer \& Senn-Irlet (2001a) & A. viridis & Switzerland & 1 \\
\hline \multirow[t]{2}{*}{ C. bolaris } & Brand (19911) & Fagus sylvatica & Germany & 1 \\
\hline & Brand (1992c) & F. sylvatica & Germany & 1 \\
\hline C. calochrous & Kernaghan (2001) & Picea engelmannii & Canada & 1 \\
\hline \multirow[t]{3}{*}{ C. cinnabarinus } & Brand (19911) & Fagus sylvatica & Germany & 1 \\
\hline & Brand $(1992 d)$ & F. sylvatica & Germany & 1 \\
\hline & Ceruti, Benvenuti \& Luppi Mosca (1988) & F. sylvatica & Italy & 1 \\
\hline C. helodes & Becerra et al. (2005) & Alnus acuminata & Argentina & 1 \\
\hline \multirow[t]{2}{*}{ C. helvelloides } & Pritsch et al. (1997) & A. glutinosa & Germany & 1 \\
\hline & Wiedmer \& Senn-Irlet (2001b) & A. viridis & Switzerland & 1 \\
\hline \multirow[t]{2}{*}{ C. hercynicus } & Agerer (1988b) & Picea abies & Germany & 1 \\
\hline & Agerer (1990c) & P. abies & Germany & 1 \\
\hline C. hinnuleus & Kovacs et al. (2002) & Betula celtiberica & Spain & 1 \\
\hline C. huronensis & Kuss, Raidl \& Beenken (2004) & Pinus rotundata & Germany & 1 \\
\hline C. ionochlorus & Montecchio et al. (2001a) & Quercus ilex & Italy & 1 \\
\hline C. kühneri & Wiedmer \& Senn-Irlet (1999d) & Alnus viridis & Switzerland & 1 \\
\hline C. laniger & Hagerman et al. (2001) & Pseudotsuga menziesii & Canada & 1 \\
\hline \multirow[t]{2}{*}{ C. magellanicus } & Palfner (2001) & Nothofagus dombeyi & Chile & 1 \\
\hline & Palfner (2002c) & N. dombeyi & Chile & 1 \\
\hline C. multiformis & Ceruti et al. (1988) & Fagus sylvatica & Italy & 1 \\
\hline C. multiformis & Luppi \& Gautero (1967) & Quercus petraea & Italy & 1 \\
\hline C. nemorensis & Ceruti et al. (1988) & Fagus sylvatica & Italy & 1 \\
\hline \multirow[t]{3}{*}{ C. obtusus } & Agerer $(1987 \mathrm{c})$ & Picea abies & Germany & 1 \\
\hline & Gronbach \& Agerer (1988) & P. abies & Germany & 1 \\
\hline & Gronbach (1988) & P. abies & Germany & 1 \\
\hline C.pluvius & Wiedmer \& Senn-Irlet (1998) & Alnus viridis & Switzerland & 1 \\
\hline C. subporphyropus & Godbout \& Fortin (1983) & A. crispa, A. rugosa & Canada & 2 \\
\hline \multirow[t]{2}{*}{ C. variecolor } & Agerer (1988b) & Picea abies & Germany & 1 \\
\hline & Agerer (1989) & P. abies & Germany & 1 \\
\hline \multirow[t]{2}{*}{ C. venetus } & Agerer (1987c) & P. abies & Germany & 1 \\
\hline & Agerer (1988a) & P. abies & Germany & 1 \\
\hline \multirow[t]{4}{*}{ Craterellus tubaeformis } & Fransson (2004a) & Quercus robur & Sweden & 1 \\
\hline & Mleczko (2002c) & Pinus sylvestris & Poland & 1 \\
\hline & Mleczko (2004c) & P. sylvestris & Poland & 1 \\
\hline & Trappe, Eberhart \& Luoma (2000) & Tsuga heterophylla & USA & 1 \\
\hline Cyclobalanopsirhiza argenteogranulosa & Haug et al. (1994) & Cyclobalanopsis glauca & Taiwan & 1 \\
\hline Dermocybe & Bradbury et al. (1998) & Pinus contorta & Canada & 1 \\
\hline
\end{tabular}


D. crocea

D. palustris

D. sanguinea

D. semisanguinea

Descolea antarctica

Descomyces albus

Elaphomyces aculeatus

E. muricatus

Entoloma sinuatum

Fagirhiza arachnoidea

F. cystidiophora

F. fusca

F. globulifera

F. granulosa

F. lanata

F. oleifera

F. pallida

F. rosea

F. setifera

F. spinulosa

F. tubulosa

F. vermiculiformis

Gautieria inapire

G. othii

Geastrum fimbriatum

Genea

G. hispidula

G. klotzschi
Uhl \& Agerer (1987)

Uhl \& Agerer (1987)

Agerer (1987a)

Agerer (1987d)

Agerer \& Uhl (1989)

Palfner (1997)

Palfner (1998b)

Palfner (2001)

Agerer, Beenken \& Bougher (2001)

Agerer (1999c)

Agerer (2002a)

Brand (1991g)

Brand (19911)

Fontana \& Centrella (1967)

Agerer (1997)

Agerer (1998a)

Brand (19911)

Brand (1992e)

Brand (1988a)

Brand (1991d)

Brand (19911)

Brand (19911)

Brand (1992a)

Brand (1988b)

Brand (19911)

Brand (19911)

Brand (19911)

Brand (19911)

Brand (1991b)

Brand (19911)

Brand (1991a)

Brand (19911)

Brand (1988c)

Jakucs \& Agerer (1998)

Jakucs (1998)

Palfner (2001)

Palfner (2002d)

Duñabeitia et al. (1996)

Agerer \& Beenken (1998a)

Agerer (1998b)

Jakucs (2002a)

Brand (1991e)

Brand (19911)

Fontana \& Centrella (1967)
Pinus sylvestris

P. sylvestris

P. mugo

Picea abies

P. abies

Pinus sylvestris

Nothofagus alpina

$N$. alpina

N. alpina

Eucalyptus

Quercus robur

Q. robur

Fagus sylvatica

F. sylvatica

Castanea sativa, F. sylvatica

Salix

Fagus sylvatica

F. sylvatica

F. sylvatica

F. sylvatica

F. sylvatica

F. sylvatica

F. sylvatica

F. sylvatica

F. sylvatica

F. sylvatica

F. sylvatica

F. sylvatica

F. sylvatica

F. sylvatica

F. sylvatica

F. sylvatica

F. sylvatica

F. sylvatica

F. sylvatica

Nothofagus pumilio

$N$. pumilio

Pinus radiata

Fagus sylvatica

F. sylvatica

Populus alba

Fagus sylvatica

F. sylvatica

Carpinus betulus, Quercus petraea
Germany

Germany

Germany

Germany

Germany

Germany

Germany

Germany

Germany

Chile

Chile

Chile

Australia

Germany

Germany

Germany

Germany

Italy

Germany

Germany

Germany

Germany

Germany

Germany

Germany

Germany

Germany

Germany

Germany

Germany

Germany

Germany

Germany

Germany

Germany

Germany

Germany

Hungary

Hungary

Chile

Chile

Spain

Germany

Germany

Hungary

Germany

Germany

Italy 
Table 1. (Cont.)

\begin{tabular}{|c|c|c|c|c|}
\hline Ectomycorrhiza & Reference & Host tree & Country & Habitat \\
\hline \multirow[t]{3}{*}{ Genea verrucosa } & De Román \& De Miguel (2005) & Quercus ilex & Spain & 1 \\
\hline & Jakucs \& Bratek (1998a) & Quercus & Hungary & 1 \\
\hline & Jakucs, Bratek \& Agerer (1998a) & Quercus & Hungary & 1 \\
\hline \multirow[t]{2}{*}{ Gomphidius glutinosus } & Agerer (1991a) & Picea abies & Germany & 1 \\
\hline & Agerer (1991b) & P. abies & Germany & 1 \\
\hline \multirow[t]{2}{*}{ G. roseus } & Agerer (1991a) & P. abies & Germany & 1 \\
\hline & Agerer (1992b) & Pinus sylvestris & Germany & 1 \\
\hline \multirow[t]{2}{*}{ Gomphus clavatus } & Agerer $(2002 b)$ & Picea abies & Germany & 1 \\
\hline & Agerer, Beenken \& Christan (1998a) & P. abies & Germany & 1 \\
\hline Gyrodon lividus & Agerer \& Treu (1993) & Alnus incana & Germany & 1 \\
\hline G. monticola & Becerra et al. (2005) & A. acuminata & Argentina & 1 \\
\hline \multirow{2}{*}{ Gyroporus cyanescens } & Agerer (1999d) & Pinus sylvestris & Germany & 1 \\
\hline & Agerer $(2002 c)$ & P. sylvestris & Germany & 1 \\
\hline \multirow[t]{12}{*}{ Hebeloma } & Águeda et al. (2001) & Quercus ilex & Spain & 4 \\
\hline & Bradbury (1998) & Pinus contorta & Canada & 1 \\
\hline & Bradbury et al. (1998) & P. contorta & Canada & 1 \\
\hline & Buée et al. (2004) & Fagus sylvatica & France & 1 \\
\hline & Danielson \& Pruden (1989) & Picea pungens & Canada & 1 \\
\hline & Godbout \& Fortin (1985) & Populus tremuloides & Canada & 3 \\
\hline & Hagerman et al. (1999) & Not specified & Canada & 1 \\
\hline & Jakucs (2002a) & Populus alba & Hungary & 1 \\
\hline & Jones et al. (1997) & Betula papyrifera, Pseudotsuga menziesii & Canada & 5 \\
\hline & Jones et al. (1997) & B. papyrifera, $P$. menziesii & Canada & 5 \\
\hline & Kernaghan (2001) & Picea engelmannii & Canada & 1 \\
\hline & Ursic \& Peterson (1997) & Pinus strobus & Canada & 3 \\
\hline H. alpinum & Giltrap (1982) & Betula & UK & 2 \\
\hline \multirow{2}{*}{ H. ammophilum } & Jakucs (2002b) & Fumana procumbens & Hungary & 1 \\
\hline & Jakucs, Magyar \& Beenken (1999) & F.procumbens & Hungary & 1 \\
\hline H. anthracophilum & Giltrap (1982) & Betula & UK & 2 \\
\hline H. calyptrosporum & Giltrap (1982) & Betula & UK & 2 \\
\hline H. circinans & Giltrap (1982) & Betula & UK & 2 \\
\hline H. claviceps & Giltrap (1982) & Betula & UK & 2 \\
\hline \multirow[t]{5}{*}{ H. crustuliniforme } & Chu-Chou \& Grace (1983) & Pseudotsuga menziesii & N. Zealand & 1 \\
\hline & Giltrap (1982) & Betula & UK & 2 \\
\hline & Godbout \& Fortin (1983) & Alnus crispa, A. rugosa & Canada & 2 \\
\hline & Miller et al. (1990) & A. rubra & USA & 5 \\
\hline & Voiry (1981) & Quercus & France & 1 \\
\hline H. cylindrosporum & Giltrap (1982) & Betula & UK & 2 \\
\hline \multirow{3}{*}{ H. edurum } & Torres \& Honrubia (1994) & Pinus halepensis & Spain & 2 \\
\hline & Treu (1990a) & Larix decidua & Germany & 1 \\
\hline & Treu (1990c) & L. decidua & Germany & 1 \\
\hline H. helodes & Kaldorf (2004) & Populus tremula $\times P$. tremuloides & Germany & 1 \\
\hline \multirow{2}{*}{ H. hiemale } & Fassi \& De Vecchi (1962) & Pinus strobus & Italy & 3 \\
\hline & Fontana (1964) & Populus & Italy & 1 \\
\hline
\end{tabular}


Fontana (1964)

Giltrap (1982)

Giltrap (1982)

Giltrap (1982)

Duñabeitia et al. (1996)

Fontana (1961)

Giltrap (1982)

Fassi \& Fontana (1966)

Ingleby et al. (1990)

Giltrap (1982)

Granetti \& Angelini (1992)

H. minus

H. pumilum

H. pusillum
H. radicosum

H. sacchariolens

H. sarcophyllum

H. sinapizans

H. cfr sinapizans

H. spoliatum

H. subsaponaceum

$H$. truncatum

$H$. velutipes

H. velutipes group

H. cortinarius

Helianthemirhiza hirsuta

Humaria hemisphaerica

H. caeruleum

Hydnellum peckii

Hydnum rufescens

Hygrophorus

H. lucorum

H. pustulatus

Hymenogaster citrinus

\section{H. hessei}

$H$. tener

Hysterangium clathroides

H. crassirhachis

H. stoloniferum

Inocybe

Giltrap (1982)

Ingleby et al. (1990)

Giltrap (1982)

Giltrap (1982)

Giltrap (1982)

Giltrap (1982)

Giltrap (1982)

Haug (2002b)

Kovacs \& Jakucs (2001)

Ingleby et al. (1990)

Kernaghan (2001)

Agerer (1993b)

Agerer (1993d)

Kraigher \& Agerer (1996)

Bradbury et al. (1998)

Kernaghan (2001)

Treu (1990a)

Gronbach (1988)

Gronbach (1989a)

Etayo \& De Miguel (1998)

Fontana \& Centrella (1967)

Fontana \& Centrella (1967)

Fontana \& Centrella (1967)

Müller \& Agerer (1996a)

Müller \& Agerer (1996c)

Raidl \& Agerer (1998a)

Azul \& Freitas (1999)
Salix purpurea

Betula

Betula

Pinus radiata

Populus

Betula

Pinus strobus

Picea sitchensis

Betula

Corylus avellana, Ostrya carpinifolia,

Quercus ilex, Q. pubescens

Betula

Betula

De Román \& De Miguel (2005)

B. pendula

Betula

Betula

Quercus ilex

Betula

Betula

Betula

De Román \& De Miguel (2005)

Agerer, Kraigher \& Javornik (1996b)

Bencivenga, Donnini \& Di Massimo (1992)

Donnini \& Bencivenga (1995)

Bradbury (1998)
Betula

Picea abies

P. abies

Quercus ilex

Helianthemum ovatum

Picea sitchensis

$P$. engelmannii

P. abies

P. abies

Picea abies

P. abies

Pinus contorta

Abies lasiocarpa, Picea engelmanii

Larix decidua

Picea abies

P. abies

Corylus avellana, Quercus pubescens

C. avellana, Populus alba, $Q$. pubescens

Not specified

Quercus petraea

$Q$. petraea

Q. petraea

Pseudotsuga menziesi

P. menziesii

Picea abies

Quercus suber

Pinus contorta

Canada

Germany

Germany

Germany

Germany

Canada

Canada

Germany

Germany

Italy

Italy

Spain

Italy

Italy

Italy

Germany

Germany

Portugal

Canada 
Table 1. (Cont.)

\begin{tabular}{|c|c|c|c|c|}
\hline Ectomycorrhiza & Reference & Host tree & Country & Habitat \\
\hline \multirow[t]{4}{*}{ Inocybe (cont.) } & Buée et al. (2004) & Fagus sylvatica & France & 1 \\
\hline & Schramm (1966) & Pinus virginiana & USA & 1 \\
\hline & Buée et al. (2004) & Fagus sylvatica & France & 1 \\
\hline & Kernaghan (2001) & Dryas octopetala & Canada & 1 \\
\hline I. albidodisca & Hagerman et al. (2001) & Pseudotsuga menziesii & Canada & 1 \\
\hline \multirow[t]{2}{*}{ I. appendiculata } & Beenken (1996a) & Picea abies & Germany & 1 \\
\hline & Beenken, Agerer \& Bahnweg (1996a) & P. abies & Germany & 1 \\
\hline I. avellana & Ingleby (1999a) & Shorea leprosula & Indonesia & 1 \\
\hline \multirow[t]{2}{*}{ I. fuscomarginata } & Beenken (1996b) & Populus nigra & Germany & 1 \\
\hline & Beenken, Agerer \& Bahnweg (1996b) & Salix & Germany & 1 \\
\hline \multirow[t]{2}{*}{ I. heimii } & Jakucs $(2002 c)$ & Fumana procumbens & Hungary & 1 \\
\hline & Magyar, Beenken \& Jakucs (1999) & F. procumbens & Hungary & 1 \\
\hline \multirow[t]{2}{*}{ I. lacera } & Cripps (1997a) & Populus tremuloides & USA & 1 \\
\hline & Ingleby et al. (1990) & Betula pendula & UK & 1 \\
\hline \multirow[t]{2}{*}{ I. obscurobadia } & Beenken $(1996 \mathrm{c})$ & Picea abies & Germany & 1 \\
\hline & Beenken, Agerer \& Bahnweg (1996c) & P. abies & Germany & 1 \\
\hline I. petiginosa & Ingleby et al. (1990) & P. sitchensis & UK & 1 \\
\hline \multirow[t]{2}{*}{ I. terrigena } & Beenken (1996d) & Pinus sylvestris & Germany & 1 \\
\hline & Beenken, Agerer \& Bahnweg (1996d) & P. sylvestris & Germany & 1 \\
\hline Ixocomus bovinus & Fassi \& De Vecchi (1962) & P. strobus & Italy & 3 \\
\hline \multirow{3}{*}{ Laccaria } & Di Battista et al. (2002) & Pseudotsuga menziesii & USA & 3 \\
\hline & Kaldorf (2004) & Populus tremula $\times P$. tremuloides & Germany & 1 \\
\hline & Kaldorf (2004) & P. tremula $\times P$. tremuloides & Germany & 1 \\
\hline \multirow[t]{2}{*}{ L. amethystina } & Brand (1988d) & Fagus sylvatica & Germany & 1 \\
\hline & Buée et al. (2004) & F. sylvatica & France & 1 \\
\hline \multirow[t]{2}{*}{ L. bicolor } & Godbout \& Fortin (1985) & Populus tremuloides & Canada & 3 \\
\hline & Weiss (1991a) & Picea abies & Germany & 2 \\
\hline \multirow[t]{9}{*}{ L. laccata } & Ceruti et al. (1988) & Fagus sylvatica & Italy & 1 \\
\hline & Chu-Chou \& Grace (1983) & Pseudotsuga menziesii & N. Zealand & 1 \\
\hline & Dickie et al. (2004) & Helianthemum bicknellii & USA & 1 \\
\hline & Fassi \& Fontana (1966) & Pinus strobus & Italy & 3 \\
\hline & Godbout \& Fortin (1983) & Alnus crispa, A. rugosa & Canada & 2 \\
\hline & Meotto (1988) & Acacia dealbata & Italy & 1 \\
\hline & Miller et al. (1990) & Alnus rubra & USA & 5 \\
\hline & Thomas \& Jackson (1979) & Picea sitchensis & UK & 3 \\
\hline & Torres et al. (1995) & Cistus ladanifer & Spain & 3 \\
\hline L. montana & Kernaghan (2001) & $\begin{array}{l}\text { Abies lasiocarpa, Dryas octopetala, Picea } \\
\text { engelmanii, Salix barrattiana }\end{array}$ & Canada & 1 \\
\hline L. proxima & Ingleby et al. (1990) & Picea sitchensis & UK & 1 \\
\hline L. tortilis & Ingleby et al. (1990) & P. sitchensis & UK & 1 \\
\hline \multirow{4}{*}{ Lactarius } & Bradbury (1998) & Pinus contorta & Canada & 1 \\
\hline & Bradbury et al. (1998) & P. contorta & Canada & 1 \\
\hline & Buée et al. (2004) & Fagus sylvatica & France & 1 \\
\hline & Buée et al. (2004) & F. sylvatica & France & 1 \\
\hline
\end{tabular}


Godbout \& Fortin (1985)

Hagerman et al. (1999)

Haug et al. (1994)

Haug et al. (2005)

Jones et al. (1997)

Jones et al. (1997)

Kaldorf (2004)

Brand (19911)

Brand (1992b)

Yamada, Ogura \& Ohmasa (2001)

Kernaghan, Currah \& Bayer (1997)

Treu (1990a)

Treu (1990d)

Treu (1990a)

Treu (1990a)

Treu (1990e)

Ceruti et al. (1988)

Buée et al. (2004)

Voiry (1981)

Kernaghan et al. (1997)

Brand (19911)

Dickie et al. (2004)

Luppi \& Gautero (1967)

Palfner (1998c)

Jakucs (2002a)

Jakucs (2002d)

Jakucs, Majoros \& Beenken (2001)

Gronbach (1988)

Bradbury et al. (1998)

Kernaghan et al. (1997)

Massicotte et al. (1999b)

Torres \& Honrubia (1994)

Agerer (1986b)

Agerer (1987e)

Münzenberger et al. (1986)

Luppi \& Gautero (1967)

Ingleby et al. (1990)

Yamada et al. (2001)

Eberhardt et al. (2000)

Wiedmer \& Senn-Irlet (2004)

Kraigher, Agerer \& Javornik (1995)

Pritsch et al. (1997)

Weiss (1991a)

Miller et al. (1990)

Pritsch et al. (1997)

Pritsch et al. (1997)

Brand (1991h)

Brand (19911)
Populus tremuloides

Not specified

Tsuga chinensis

Betula papyrifera, Pseudotsuga menziesii

B. papyrifera, P. menziesii

Populus tremula $\times$ P. tremuloides

Fagus sylvatica

F. sylvatica

Pinus densiflora

Picea engelmannii

Alnus viridis

A. viridis

Picea abies

Pinus cembra

P. cembra

Fagus sylvatica

F. sylvatica

Fagus

Abies lasiocarpa

Fagus sylvatica

Helianthemum bicknellii

Quercus petraea

Q. robur

Populus alba

P. alba

P. alba

Picea abies

Pinus contorta

Abies lasiocarpa

Pinus ponderosa, Pseudotsuga menziesi

P. halepensis

Picea abies

P. abies

P. abies

Quercus pubescens

Betula pendula

Pinus densiflora

Abies alba

Alnus viridis

Picea abies

Alnus glutinosa

Picea abies

Alnus rubra

A. glutinosa

A. glutinosa

Fagus sylvatica

F. sylvatica
Canada

Canada

Taiwan

Ecuador

Canada

Canada

Germany

Germany

Germany

Japan

Canada

Germany

Germany

Germany

Germany

Germany

Italy

France

France

Canada

Germany

USA

Italy

Germany

Hungary

Hungary

Germany

Canada

Canad

USA

Spain

Germany

Germany

Italy

UK

Japan

Italy

Switzerland

Slovenia

Germany

Germany

USA

Germany

Germany

Germany

Germany 
Table 1. (Cont.)

\begin{tabular}{|c|c|c|c|c|}
\hline Ectomycorrhiza & Reference & Host tree & Country & Habitat \\
\hline Lactarius paradoxus & Danielson (1984b) & Pinus banksiana & Canada & 2 \\
\hline \multirow{2}{*}{ L. picinus } & Agerer (1986b) & Picea abies & Germany & 1 \\
\hline & Agerer (1987f) & P. abies & Germany & 1 \\
\hline L.piperatus & Luppi \& Gautero (1967) & Quercus petraea & Italy & 1 \\
\hline \multirow[t]{2}{*}{ L. porninsis } & Treu (1990a) & Larix decidua & Germany & 1 \\
\hline & Treu (1990f) & L. decidua & Germany & 1 \\
\hline L. pterosporus & Ceruti et al. (1988) & Fagus sylvatica & Italy & 1 \\
\hline L. pubescens & Ingleby et al. (1990) & Betula pendula & UK & 1 \\
\hline L. rubrilacteus & Eberhart \& Luoma (1997) & Pseudotsuga menziesii & USA & 1 \\
\hline \multirow[t]{2}{*}{ L. rubrocinctus } & Brand (1991i) & Fagus sylvatica & Germany & 1 \\
\hline & Brand (19911) & F. sylvatica & Germany & 1 \\
\hline \multirow[t]{2}{*}{ L. rufus } & Ingleby et al. (1990) & Picea sitchensis & UK & 1 \\
\hline & Wöllecke (2001) & Pinus sylvestris & Germany & 1 \\
\hline \multirow[t]{2}{*}{ L. salmonicolor } & Eberhardt et al. (2000) & Abies alba & Italy & 1 \\
\hline & Pillukat (1996) & A. alba & Germany & 1 \\
\hline L. salmonicolor & Pillukat (1998) & A. alba & Germany & 1 \\
\hline L. scrobiculatus & Kernaghan \& Berch (1997) & Tsuga heterophylla & Canada & 1 \\
\hline \multirow{2}{*}{ L. subdulcis } & Brand (1987a) & Fagus sylvatica & Germany & 1 \\
\hline & Buée et al. (2004) & F. sylvatica & France & 1 \\
\hline L. subsericatus & Eberhardt et al. (2000) & Abies alba & Italy & 1 \\
\hline L. tesquorum & Nuytinck et al. (2004) & Cistus & Italy & 1 \\
\hline L. theiogalus & Gronbach (1988) & Picea abies & Germany & 1 \\
\hline \multirow[t]{3}{*}{ L. vellereus } & Brand (1987b) & Fagus sylvatica & Germany & 1 \\
\hline & Ceruti et al. (1988) & F. sylvatica & Italy & 1 \\
\hline & Luppi \& Gautero (1967) & Quercus petraea & Italy & 1 \\
\hline \multirow{3}{*}{ Laricirhiza alpina } & Hagerman et al. (2001) & Pseudotsuga menziesii & Canada & 1 \\
\hline & Treu (1990a) & Larix decidua & Germany & 1 \\
\hline & Treu $(1990 \mathrm{~g})$ & L. decidua & Germany & 1 \\
\hline \multirow[t]{3}{*}{ Leccinum } & Bradbury et al. (1998) & Pinus contorta & Canada & 1 \\
\hline & Godbout \& Fortin (1985) & Populus tremuloides & Canada & 3 \\
\hline & Ingleby et al. (1990) & Betula pendula & UK & 1 \\
\hline L. holopus & Godbout \& Fortin (1983) & Alnus crispa, A. rugosa & Canada & 2 \\
\hline L. scabrum & Müller \& Agerer (1991) & Betula pendula & Germany & 1 \\
\hline L. subleucophaeum & Godbout \& Fortin (1983) & Alnus crispa, A. rugosa & Canada & 2 \\
\hline \multirow[t]{2}{*}{ Leucangium carthusianum } & Palfner \& Agerer (1998b) & Pseudotsuga menziesii & USA & 1 \\
\hline & Palfner (1998d) & P. menziesii & USA & 1 \\
\hline \multirow[t]{2}{*}{ Lyophyllum decastes } & Agerer \& Beenken (1998b) & Quercus robur & Germany & 1 \\
\hline & Agerer $(1998 c)$ & Q. robur & Germany & 1 \\
\hline L. semitale & Yamada et al. (2001) & Pinus densiflora & Japan & 5 \\
\hline L. shimeji & Yamada et al. (2001) & P. densiflora & Japan & 5 \\
\hline Melanogaster broomeianus & Wiedmer et al. (2004) & Alnus viridis & Switzerland & 1 \\
\hline M. variegatus & Giraud (1988) & Not specified & France & 4 \\
\hline \multirow[t]{2}{*}{ Naucoria escharoides } & Becerra et al. (2002) & Alnus acuminata & Argentina & 1 \\
\hline & Pritsch et al. (1997) & A. glutinosa & Germany & 1 \\
\hline
\end{tabular}


N. subconspersa

Nothofagirhiza reticulosa

$N$. tricystidiis

$N$, vesiculosa

$N$. vinicolor

Pasanirhiza strigosa

Paxillus

P. involutus

P. involutus

\section{Pezizaceae}

\section{Pezizalean}

Pezizales

Phellodon niger

Phialocephala fortinii

Piceirhiza bicolorata

P. chordata

P. conspicua

P. cornuta

P. cortinacearum

P. gelatinosa

P. glutinosa

P. guttata

P. horti-atrata

P. internicrassihyphis

P. nigra

P. obscura

P. oleiferans

P. rufosimilis

P. stagonopleres

P. tomentellarum
Pritsch et al. (1997)

Palfner (2001)

Palfner (2001)

Palfner (2001)

Palfner (2001)

Palfner \& Godoy (1996a)

Palfner \& Godoy (1996c)

Haug et al. (1994)

Jones et al. (1997)

Agerer \& Gronbach (1989)

Godbout \& Fortin (1983)

Godbout \& Fortin (1985)

Gronbach (1988)

Ingleby et al. (1990)

Miller et al. (1990)

Mleczko (1997a)

Buée et al. (2004)

Buée et al. (2004)

Dickie et al. (2004)

Kaldorf (2004)

Kaldorf (2004)

Kaldorf (2004)

Agerer (1993c)

Kaldorf (2004)

Brand, Gronbach \& Taylor (1992)

Gronbach (1988)

Gronbach (1988)

Gronbach (1989b)

Gronbach (1988)

Gronbach (1989c)

Montecchio \& Agerer (1997)

Haug (2002b)

Berg \& Gronbach (1989)

Gronbach (1988)

Gronbach (1988)

Gronbach (1989d)

Gronbach (1988)

Gronbach (1989e)

Al Sayegh \& Kraigher (1999)

Agerer (2002e)

Berg \& Gronbach (1988)

Gronbach (1988)

Gronbach \& Berg (1988)

Gronbach (1988)

Waller et al. (1993)

Gronbach (1988)

Beenken \& Agerer (1996)

Haug (2002b)
A. glutinosa

Nothofagus pumilio

N. oblicua

N. dombeyi

N. pumilio

N. pumilio

N. pumilio

Pasania glabra

Betula papyrifera, Pseudotsuga menziesii

Picea abies

Alnus crispa, A. rugos

Populus tremuloides

Picea abies

Betula pendula

Alnus rubra

Pinus sylvestris

Fagus sylvatica

F. sylvatica

Helianthemum bicknellii

Populus tremula $\times P$. tremuloides

$P$. tremula $\times P$. tremuloides

$P$. tremula $\times P$. tremuloides

Picea abies

Populus tremula $\times$ P. tremuloides

Picea abies

P. abies

P. abies

P. abies

P. abies

P. abies

P. abies

P. abies

P. abies

P. abies

P. abies

P. abies
P. abies

P. abies
P. abies

P. abies

P. abies

P. abies

P. abies

P. abies

P. abies

P. abies

P. abies

P. abies

P. abies 
Table 1. (Cont.)

\begin{tabular}{|c|c|c|c|c|}
\hline Ectomycorrhiza & Reference & Host tree & Country & Habitat \\
\hline Picoa lefebvrei & Gutierrez, Morte \& Honrubia (2003) & Helianthemum almeriense & Spain & 2 \\
\hline \multirow[t]{3}{*}{ Piloderma } & Hagerman et al. (1999) & Not specified & Canada & 1 \\
\hline & Hagerman et al. (2001) & Pseudotsuga menziesii & Canada & 1 \\
\hline & Massicotte et al. (1999b) & Pinus ponderosa & USA & 3 \\
\hline \multirow[t]{2}{*}{ P. byssinum } & Bradbury (1998) & P. contorta & Canada & 1 \\
\hline & Bradbury et al. (1998) & P. contorta & Canada & 1 \\
\hline \multirow[t]{2}{*}{ P. croceum } & Brand $(1991 \mathrm{j})$ & Fagus sylvatica & Germany & 1 \\
\hline & Brand (19911) & F. sylvatica & Germany & 1 \\
\hline \multirow[t]{4}{*}{ P. fallax } & Bradbury (1998) & Pinus contorta & Canada & 1 \\
\hline & Bradbury et al. (1998) & P. contorta & Canada & 1 \\
\hline & Goodman \& Trofymow (1996) & Pseudotsuga menziesii & Canada & 1 \\
\hline & Kernaghan (2001) & Picea engelmannii & Canada & 1 \\
\hline Pinirhiza aciculata & Haug et al. (1994) & Pinus taiwanensis & Taiwan & 1 \\
\hline \multirow[t]{2}{*}{ P. amyloidea } & Mleczko (2002d) & P. sylvestris & Poland & 1 \\
\hline & Mleczko (2004d) & P. sylvestris & Poland & 1 \\
\hline \multirow[t]{2}{*}{ P. arachnorosea } & Wöllecke, Münzenberger \& Hüttl (1997a) & P. sylvestris & Germany & 1 \\
\hline & Wöllecke, Münzenberger \& Hüttl (1998c) & P. sylvestris & Germany & 1 \\
\hline P. cephalocystidia & Wöllecke, Münzenberger \& Hüttl (1999a) & P. sylvestris & Germany & 1 \\
\hline \multirow[t]{2}{*}{ P. crystallonitida } & Mleczko (2002e) & P. sylvestris & Poland & 1 \\
\hline & Mleczko (2004e) & P. sylvestris & Poland & 1 \\
\hline \multirow[t]{2}{*}{ P. cyaneoviridis } & Golldack, Münzenberger \& Hüttl (1998a) & P. sylvestris & Germany & 1 \\
\hline & Golldack, Münzenberger \& Hüttl (1998c) & P. sylvestris & Germany & 1 \\
\hline \multirow[t]{2}{*}{ P. dimorpha } & De Román \& De Miguel (2005) & Quercus ilex & Spain & 1 \\
\hline & Golldack, Münzenberger \& Hüttl (1999a) & Pinus sylvestris & Germany & 1 \\
\hline \multirow[t]{2}{*}{ P. discolor } & Golldack, Münzenberger \& Hüttl (1998b) & P. sylvestris & Germany & 1 \\
\hline & Golldack, Münzenberger \& Hüttl (1998d) & P. sylvestris & Germany & 1 \\
\hline \multirow[t]{2}{*}{ P. echinata } & Haug et al. (1994) & P. taiwanensis & Taiwan & 1 \\
\hline & Wöllecke (2001) & P. sylvestris & Germany & 1 \\
\hline P. epidermoides & Haug et al. (1994) & P. taiwanensis & Taiwan & 1 \\
\hline P. flexipila & Golldack, Münzenberger \& Hüttl (1999b) & P. sylvestris & Germany & 1 \\
\hline P. globulifera & Mleczko (1998a) & P. sylvestris & Poland & 1 \\
\hline \multirow[t]{2}{*}{ P. gomphidioidea } & Mleczko (1998b) & P. sylvestris & Poland & 1 \\
\hline & Mleczko (1998c) & P. sylvestris & Poland & 1 \\
\hline \multirow[t]{2}{*}{ P. granulosa } & Golldack et al. (1996a) & P. sylvestris & Germany & 1 \\
\hline & Golldack, Münzenberger \& Hüttl (1996c) & P. sylvestris & Germany & 1 \\
\hline P. hyphocystidia & Wöllecke, Münzenberger \& Hüttl (1999b) & P. sylvestris & Germany & 1 \\
\hline P. lactariosimilis & Golldack, Münzenberger \& Hüttl (1997a) & P. sylvestris & Germany & 1 \\
\hline P. lactogelatinosa & Golldack, Münzenberger \& Hüttl (1997b) & P. sylvestris & Germany & 1 \\
\hline \multirow[t]{2}{*}{ P. ligulata } & Mleczko (2002f) & P. sylvestris & Poland & 1 \\
\hline & Mleczko (2004f) & P. sylvestris & Poland & 1 \\
\hline \multirow[t]{2}{*}{ P. lutea } & Mleczko (1996a) & P. sylvestris & Poland & 1 \\
\hline & Mleczko (1996b) & P. sylvestris & Poland & 1 \\
\hline & Wöllecke, Münzenberger \& Hüttl (1997b) & P. sylvestris & Germany & 1 \\
\hline \multirow[t]{2}{*}{ P. luteomicacea } & Mleczko $(2002 \mathrm{~g})$ & P. sylvestris & Poland & 1 \\
\hline & Mleczko (2004g) & P. sylvestris & Poland & 1 \\
\hline
\end{tabular}


Golldack et al. (

P. subalpina

P. sulphurea

Pisolithus

P. aurantioscabrosus

P. tinctorius

Polyporoletus sublividus

Populirhiza pustulosa

Pseudotomentella tristis

Pseudotsugaerhiza baculifera

Pulvinulla constellatio

Quercirhiza alboviolacea

Q. araneosa

Q. argenteobrunneola

Q. atrata

O. bicolor

Q. cistidiophora

Q. cumulosa

Q. ectendotrophica

Q. fibulocystidiata

Q. incrustata

Q. internangularis

Q. nodulosomorpha

Q. pedicae

Q. russulocystidiata

Q. sclerotiigera

Q. squamosa
Treu (1990a)

Wöllecke, Münzenberger \& Hüttl (1998b)

Águeda et al. (2001)

Moyersoen \& Beever (2004)

Moyersoen \& Beever (2004)

Watling et al. (1995a)

Watling et al. (1995b)

De Román \& De Miguel (2005)

Godbout \& Fortin (1983)

Godbout \& Fortin (1985)

Rose et al. (1981)

Torres \& Honrubia (1994)

Weiss (1991b)

Weiss (1992)

Agerer \& Ammirati (1998)

Agerer, Beenken \& Ammirati (1998b)

Mleczko (1997b)

Mleczko (1998d)

Agerer (1994b)

Müller \& Agerer (1996b)

Müller \& Agerer (1996d)

Amicucci et al. (2001)

Bencivenga et al. (1995)

Jakucs (2001)

Jakucs (2002e)

Montecchio et al. (1999a)

Fischer \& Agerer (1996)

Al Sayegh \& Kraigher (1999)

Montecchio et al. (1999b)

Montecchio et al. (2004)

De Román \& De Miguel (2005)

De Román, Agerer \& De Miguel (2002a)

Azul, Agerer \& Freitas (2001a)

Jakucs, Agerer \& Bratek (1997)

Jakucs, Agerer \& Bratek (1998c)

Montecchio, Rossi \& Causin (2001b)

Azul, Agerer \& Freitas (2001b)

Azul \& Freitas (1999)

Azul, Agerer \& Freitas (1999)

Azul, Agerer \& Freitas (2001c)

Azul, Agerer \& Freitas (2001d)

Azul, Agerer \& Freitas (2001e)

Palfner (1995a)
P. sylvestris

P. cembra

P. sylvestris

Quercus ilex

Kunzea ericoides

Leptospermum scoparium

Shorea parviflora

S. parviflora

Quercus ilex

Alnus crispa, A. rugosa

Populus tremuloides

Eucalyptus nova-anglica

Pinus halepensis

Picea abies

P. abies

Abies amabilis

A. amabilis

Populus tremula

P. tremula

Salix herbacea

Pseudotsuga menziesii

P. menziesii

Quercus pubescens

Not specified

Quercus robur

Q. robur

Q. ilex

Q. ilex

Picea abies

Quercus ilex

$Q$. ilex

$Q$. ilex

Q. ilex

Q. suber

Quercus

Quercus

Q. ilex

$Q$. suber

Q. suber

Q. suber

Q. suber

$Q$. suber

Q. suber

Q. robur 
Table 1. (Cont.)

\begin{tabular}{|c|c|c|c|c|}
\hline Ectomycorrhiza & Reference & Host tree & Country & Habitat \\
\hline & Palfner \& Agerer (1996) & Q. robur & Slovenia & 1 \\
\hline Quercirhiza stellata & De Román, Agerer \& De Miguel (2002b) & Quercus ilex & Spain & 1 \\
\hline \multirow[t]{2}{*}{ Q. sublutea } & Montecchio et al. (1998a) & Q. ilex & Italy & 1 \\
\hline & Montecchio et al. (1998b) & Q.ilex & Italy & 1 \\
\hline Ramaria & Nouhra et al. (2005) & Pseudotsuga menziesii, Tsuga heterophylla & USA & 1 \\
\hline R. aurea & Agerer (1996c) & Fagus sylvatica & Germany & 1 \\
\hline \multirow[t]{2}{*}{ R. largentii } & Agerer (1996d) & Picea abies & Germany & 1 \\
\hline & Agerer (1996h) & P. abies & Germany & 1 \\
\hline R. spinulosa & Agerer (1996e) & Fagus sylvatica & Germany & 1 \\
\hline \multirow{2}{*}{ R. subbotrytis } & Agerer (1996f) & Quercus robur & Germany & 1 \\
\hline & Agerer (1998d) & Q. robur & Germany & 1 \\
\hline \multirow[t]{7}{*}{ Rhizopogon } & Al Sayegh \& Kraigher (2000) & Fagus & Slovenia & 1 \\
\hline & Chu-Chou \& Grace (1983) & Pseudotsuga menziesii & N. Zealand & 1 \\
\hline & Di Battista et al. (2002) & P. menziesii & USA & 3 \\
\hline & Hagerman et al. (2001) & P. menziesii & Canada & 1 \\
\hline & Jones et al. (1997) & P. menziesii & Canada & 5 \\
\hline & Torres \& Honrubia (1997) & Pinus halepensis & Spain & 3 \\
\hline & Danielson et al. (1984) & P. banksiana & Canada & 3 \\
\hline R. arctostaphyli & Massicotte et al. (1999a) & P. ponderosa & Canada & 3 \\
\hline R. ellenae & Massicotte et al. (1999a) & P. ponderosa & Canada & 3 \\
\hline R. flavofibrillosus & Massicotte et al. (1999a) & P. ponderosa & Canada & 3 \\
\hline \multirow{2}{*}{ R. luteolus } & Mohan, Natarajan \& Ingleby (1993) & P. patula & India & 3 \\
\hline & Uhl (1988b) & P. sylvestris & Germany & 1 \\
\hline \multirow[t]{2}{*}{ R. melanogastroides } & Raidl (1998a) & P. mugo & Germany & 1 \\
\hline & Raidl, Beenken \& Agerer (1998) & P. mugo & Germany & 1 \\
\hline R. occidentalis & Massicotte et al. (1999a) & P. ponderosa & Canada & 3 \\
\hline R. parksii & Massicotte et al. (2000) & Pseudotsuga menziesii & Canada & 3 \\
\hline \multirow[t]{2}{*}{ R. roseolus } & Raidl \& Agerer (1998b) & Pinus sylvestris & Germany & 1 \\
\hline & Torres \& Honrubia (1994) & P. halepensis & Spain & 2 \\
\hline \multirow[t]{3}{*}{ R. rubescens } & Fontana \& Centrella (1967) & P. sylvestris & Italy & 1 \\
\hline & Massicotte et al. (1999b) & Arbutus menziesii, Pinus ponderosa & USA & 3 \\
\hline & Yamada et al. (2001) & P. densiflora & Japan & 5 \\
\hline \multirow[t]{4}{*}{ R. subcaerulescens } & Agerer (1996i) & Tsuga heterophylla & Germany & 1 \\
\hline & Agerer, Müller \& Bahnweg (1996c) & T. heterophylla & Germany & 1 \\
\hline & Massicotte et al. (1999a) & Pinus ponderosa & Canada & 3 \\
\hline & Massicotte et al. (2000) & Pseudotsuga menziesii & Canada & 3 \\
\hline \multirow[t]{2}{*}{ R. truncatus } & Massicotte et al. (1999a) & Pinus ponderosa & Canada & 3 \\
\hline & Massicotte et al. (1999b) & P. ponderosa & USA & 3 \\
\hline \multirow[t]{3}{*}{ R. vinicolor } & Chu-Chou \& Grace (1983) & Pseudotsuga menziesii & N. Zealand & 1 \\
\hline & Massicotte et al. (2000) & P. menziesii & Canada & 3 \\
\hline & Zak (1971) & P. menziesii & USA & 1 \\
\hline R. vinicolor-like & Goodman (1996e) & P. menziesii & Canada & 1 \\
\hline \multirow[t]{2}{*}{ R. vulgaris } & Jakucs \& Bratek (1998b) & Pinus nigra & Hungary & 1 \\
\hline & Jakucs et al. (1998b) & P. nigra & Hungary & 1 \\
\hline \multirow[t]{2}{*}{ Rhodocollybia butyracea } & Mleczko (2002h) & P. sylvestris & Poland & 1 \\
\hline & Mleczko (2004h) & P. sylvestris & Poland & 1 \\
\hline
\end{tabular}


Buée et al. (2004)

Buée et al. (2004)

Jones et al. (1997)

Jones et al. (1997)

Massicotte et al. (1999b)

\section{R. acrifolia \\ $R$. aeruginea \\ $R$. aff. amoenolens
$R$. aff. pectinatoides \\ R. alnetorum}

$R$. amoenolens

\section{R. atroglauca}

R. brevipes

R. cyanoxantha

R. decoloran

R. delica

R. densifolia

R. firmula

R. foetens

R. fuegiana

R. illota

R. integra

R. laricina

R. mairei
Agerer (1996j)

Beenken (2001a)

Dickie et al. (2004)

Dickie et al. (2004)

Beenken (2001b)

Jakucs \& Beenken (1999)

Jakucs (2002a)

Jakucs (2002a)

Jakucs (2002f)

Beenken (2001c)

Kernaghan et al. (1997)

Beenken (2001d)

Fransson (2004b)

Beenken (2001e)

Yamada (1998a)

Beenken (2001f)

Beenken (2001g)

Ceruti et al. (1988)

Eberhart \& Luoma (2000)

Treu (1990a)

Treu (1990h)

Beenken (2001h)

Palfner (2001)

Palfner \& Godoy (1996b)

Palfner \& Godoy (1996d)

Brand (1991k)

Brand (19911)

Kernaghan (2001)

Treu (1990a)

Treu (1990i)

Brand (1991c)

Brand (19911)
Shorea leprosula

Malaysia

S. leprosula

Pinus sylvest is
P. sylvestris

Quercus suber

Pinus contorta

P. contorta

Fagus sylvatica

F. sylvatica

F. sylvatica

Betula papyrifera

B. papyrifera, Pseudotsuga menziesii

Lithocarpus densifora, Pinus ponderosa

P. menziesii

Picea abies

Betula pendula

Helianthemum bicknellii

H. bicknellii

Alnus viridis

Populus alba

P. alba

P. alba

P. alba

Betula pubescens

Abies lasiocarpa

Fagus sylvatica

Pinus sylvestris

Tilia

Betula platyphylla

Fagus sylvatica

Picea abies

Fagus sylvatica

Pseudotsuga menziesi

Pinus mugo

P. mugo

Fagus sylvatica

Nothofagus pumilio

$N$. pumilio

$N$. pumilio

Fagus sylvatica

Fagus sylvatica

Abies lasiocarpa

Larix decidua

L. decidua

Fagus sylvatica

F. sylvatica 
Table 1. (Cont.)

\begin{tabular}{|c|c|c|c|c|}
\hline Ectomycorrhiza & Reference & Host tree & Country & Habitat \\
\hline Russula medullata & Beenken (2001i) & Populus tremula & Germany & 1 \\
\hline \multirow[t]{4}{*}{ R. nigricans } & Hagerman et al. (2001) & Pseudotsuga menziesii & Canada & 1 \\
\hline & Mleczko (2002i) & Pinus sylvestris & Poland & 1 \\
\hline & Mleczko (2004i) & P. sylvestris & Poland & 1 \\
\hline & Yamada (1998b) & Betula platyphylla & Japan & 1 \\
\hline R. nothofaginea & Beenken (2001j) & Nothofagus dombeyi & Germany & 1 \\
\hline \multirow{5}{*}{ R. ochroleuca } & Agerer (1986b) & Picea abies & Germany & 1 \\
\hline & Agerer (1987g) & P. abies & Germany & 1 \\
\hline & Brand (1991l) & Fagus sylvatica & Germany & 1 \\
\hline & Gronbach (1988) & Picea abies & Germany & 1 \\
\hline & Wöllecke (2001) & Pinus sylvestris & Germany & 1 \\
\hline R. puiggarii & Haug et al. (2005) & Neea & Ecuador & 1 \\
\hline R. pumila & Pritsch et al. (1997) & Alnus glutinosa & Germany & 1 \\
\hline$R$. sanguinea & Duñabeitia et al. (1996) & Pinus radiata & Spain & 3 \\
\hline \multirow[t]{2}{*}{ R. sardonia } & Mleczko (2002j) & P. sylvestris & Poland & 1 \\
\hline & Mleczko $(2004 \mathrm{j})$ & P. sylvestris & Poland & 1 \\
\hline R. silvicola & Kernaghan et al. (1997) & Abies lasiocarpa & Canada & 1 \\
\hline R. versicolor & Beenken $(2001 \mathrm{k})$ & Betula pubescens & Germany & 1 \\
\hline \multirow[t]{2}{*}{ R. vesca } & Beenken (20011) & Quercus robur & Germany & 1 \\
\hline & Luppi \& Gautero (1967) & Q. petraea & Italy & 1 \\
\hline$R$. vinosa & Beenken $(2001 \mathrm{~m})$ & Picea abies & Germany & 1 \\
\hline R. violascens & Luppi \& Gautero (1967) & Ouercus petraea & Italy & 1 \\
\hline$R$. virescens & Beenken $(2001 \mathrm{n})$ & Q. robur & Germany & 1 \\
\hline \multirow[t]{2}{*}{ R. xerampelina } & Agerer (1986b) & Picea abies & Germany & 1 \\
\hline & Agerer $(1987 \mathrm{~h})$ & P. abies & Germany & 1 \\
\hline Russulaceae & See et al. (1997) & Shorea leprosula & Malaysia & 1 \\
\hline Russula-like & Goodman (1997b) & Tsuga heterophylla & Canada & 1 \\
\hline Sarcodon imbricatus & Agerer (1991c) & Picea abies & Germany & 1 \\
\hline \multirow[t]{2}{*}{ SB (spinules buclées, Giraud 1988) } & Giraud (1988) & Not specified & France & 4 \\
\hline & Sáez \& De Miguel (1995) & Not specified & Spain & 4 \\
\hline \multirow[t]{3}{*}{ Scleroderma } & Buée et al. (2004) & Fagus sylvatica & France & 1 \\
\hline & Godbout \& Fortin (1985) & Populus tremuloides & Canada & 3 \\
\hline & See et al. (1997) & Shorea leprosula & Malaysia & 1 \\
\hline S. areolatum & Meotto, Pellegrino \& Craddock (1994) & Castanea sativa & Italy & 3 \\
\hline S. aurantium & Voiry (1981) & Quercus & France & 1 \\
\hline \multirow[t]{3}{*}{ S. bovista } & Jakucs \& Agerer (1999a) & Populus alba & Hungary & 1 \\
\hline & Jakucs (2002a) & P. alba & Hungary & 1 \\
\hline & Jakucs $(2002 \mathrm{~g})$ & P. alba & Hungary & 1 \\
\hline \multirow[t]{5}{*}{ S. citrinum } & Brunner et al. (1992) & Picea abies & Switzerland & 2 \\
\hline & Godbout \& Fortin (1983) & Alnus crispa, A. rugosa & Canada & 2 \\
\hline & Mohan et al. (1993) & Pinus patula & India & 3 \\
\hline & Richter \& Bruhn (1990) & Larix decidua & USA & 2 \\
\hline & Waller \& Agerer (1993) & Betula pendula & Germany & 1 \\
\hline S. geaster & Rose et al. (1981) & Eucalyptus nova-anglica & USA & 1 \\
\hline
\end{tabular}


S. incrustans

Sebacinaceae

\section{Sebacinoid}

Sphaerosporella brunnea

Stephanopus stropharioides Suillus

S. bovinus

S. brevipes

S. caerulescens
S. collinitus

S. flavus

S. granulatus

S. laricinus

S. luteus

S. plorans

S. sibiricus

S. tomentosus

S. tridentinus

S. umbonatus

S. variegatus

Terfezia claveryi

Tetraberliniaerhiza bicolor

T. cerviformis

T. heterocystidiae

Thaxterogaster albocanus

Thelephora
De Román \& De Miguel (2005)

Urban, Weiss \& Bauer (2003)

Selosse et al. (2002)

Selosse et al. (2002)

Urban et al. (2003)

Bencivenga et al. (1995)

Danielson (1984a)

De Román \& De Miguel (2005)

Di Massimo et al. (1996)

Meotto \& Carraturo (1988)

Palfner (2001)

Torres \& Honrubia (1997)

Yamada et al. (2001)

Bradbury et al. (1998)

Hoch, Goodman \& Chyplyk (1998)

Torres \& Honrubia (1994)

Treu (1990a)

Treu (1990j)

Yamada et al. (2001)

Treu (1990a)

Treu (1993)

Torres \& Honrubia (1994)

Yamada et al. (2001)

Treu (1990a)

Treu (1990k)

Treu (1990a)

Treu (19901)

Bradbury (1998)

Bradbury et al. (1998)

Danielson (1984b)

Treu (1990a)

Bradbury (1998)

Bradbury et al. (1998)

Torres \& Honrubia (1994)

Gutierrez et al. (2003)

Moyersoen (1996a)

Moyersoen (1996d)

Moyersoen (1996b)

Moyersoen (1996e)

Moyersoen (1996c)

Moyersoen (1996f)

Palfner (1996a)

Palfner (1996b)

Palfner (2001)

Di Battista et al. (2002)

Jones et al. (1997)
Gnetum africanum

Quercus ilex

Picea abies

Carpinus

Corylus

Tilia

Not specified

Pinus banksiana

Quercus ilex

Q. ilex

Populus nigra

Nothofagus dombeyi

Pinus halepensis

P. densiflora

P. contorta

Pseudotsuga menziesi

Pinus halepensis

Larix decidu

L. decidua

Pinus densifora

Larix decidu

Pinus halepensis

P. densiflor

P. cembra

P. cembra

P. cembra

P. cembra

P. contorta
$P$. contorta

P. banksiana

Larix decidua

Pinus contorta

P. contorta

P. halepensis

Helianthemum almeriense

Tetraberlinia bifoliolata

T. bifoliolata

T. bifoliolata

T. bifoliolata

T. bifoliolata

T. bifoliolata

Nothofagus pumilio

$N$. pumilio

N. pumilio

Pseudotsuga menziesii

Betula papyrifera, Pseudotsuga menziesii
Cameroon

Austri

France

Austria

Italy

Canad

Spain

Spain

Chile
Spain

Japan

Canada

Canada

Spain

Germany

Germany

Japan

Germany

Germany

Spain

Japan

Germany

Germany

Germany

Germany

Canada

Canada

Canada

Germany

Canada

Canada

Spain

Spain

Cameroon

Cameroon

Cameroon

Cameroon

Cameroon

Cameroo

Chile

Chile

Chile

USA 
Table 1. (Cont.)

\begin{tabular}{|c|c|c|c|c|}
\hline Ectomycorrhiza & Reference & Host tree & Country & Habitat \\
\hline Thelephora (cont.) & Massicotte et al. (1999b) & $\begin{array}{l}\text { Abies grandis, Lithocarpus densiflora, } \\
\text { Pinus ponderosa, Pseudotsuga menziesii }\end{array}$ & USA & 3 \\
\hline \multirow[t]{10}{*}{ T. terrestris } & Agerer \& Weiss (1989) & Picea abies & Germany & 1 \\
\hline & Agerer \& Weiss (1990) & P. abies & Germany & 1 \\
\hline & Chu-Chou \& Grace (1983) & Pseudotsuga menziesii & N. Zealand & 1 \\
\hline & Fassi \& Fontana (1966) & Pinus strobus & Italy & 3 \\
\hline & Godbout \& Fortin (1985) & Populus tremuloides & Canada & 3 \\
\hline & Ingleby et al. (1990) & Picea sitchensis & UK & 1 \\
\hline & Miller et al. (1990) & Alnus rubra & USA & 5 \\
\hline & Schramm (1966) & Pinus virginiana & USA & 1 \\
\hline & Thomas \& Jackson (1979) & Picea sitchensis & UK & 3 \\
\hline & Ursic \& Peterson (1997) & Pinus strobus & Canada & 3 \\
\hline T. terrestris-like & Gillespie, Durall \& Hagerman (2000) & Betula papyrifera & Canada & 1 \\
\hline \multirow[t]{10}{*}{ Thelephoraceae } & Haug et al. (2005) & Guapira & Ecuador & 1 \\
\hline & Haug et al. (2005) & Neea & Ecuador & 1 \\
\hline & Haug et al. (2005) & Neea & Ecuador & 1 \\
\hline & Kaldorf (2004) & Populus tremula $\times P$. tremuloides & Germany & 1 \\
\hline & Kaldorf (2004) & P. tremula $\times P$. tremuloides & Germany & 1 \\
\hline & Kernaghan (2001) & Abies lasiocarpa, Picea engelmanii & Canada & 1 \\
\hline & Kernaghan (2001) & A. lasiocarpa, Salix barrattiana & Canada & 1 \\
\hline & Kernaghan (2001) & Picea engelmanii, $S$. barrattiana & Canada & 1 \\
\hline & Selosse et al. (2002) & Carpinus & France & 1 \\
\hline & Selosse et al. (2002) & Carpinus & France & 1 \\
\hline \multirow[t]{21}{*}{ Tomentella } & Bradbury (1998) & Pinus contorta & Canada & 1 \\
\hline & Bradbury et al. (1998) & P. contorta & Canada & 1 \\
\hline & Buée et al. (2004) & Fagus sylvatica & France & 1 \\
\hline & Buée et al. (2004) & F. sylvatica & France & 1 \\
\hline & Buée et al. (2004) & F. sylvatica & France & 1 \\
\hline & Buée et al. (2004) & F. sylvatica & France & 1 \\
\hline & Buée et al. (2004) & F. sylvatica & France & 1 \\
\hline & Buée et al. (2004) & F. svlvatica & France & 1 \\
\hline & Buée et al. (2004) & F. sylvatica & France & 1 \\
\hline & Buée et al. (2004) & F. sylvatica & France & 1 \\
\hline & Buée et al. (2004) & F. sylvatica & France & 1 \\
\hline & Buée et al. (2004) & F. sylvatica & France & 1 \\
\hline & Buée et al. (2004) & F. sylvatica & France & 1 \\
\hline & Danielson \& Pruden (1989) & Picea pungens & Canada & 1 \\
\hline & Danielson et al. (1984) & Pinus banksiana & Canada & 3 \\
\hline & Dickie et al. (2004) & Helianthemum bicknellii & USA & 1 \\
\hline & Jakucs et al. (2004) & Populus alba & Hungary & 1 \\
\hline & Jakucs et al. (2005) & P. alba & Hungary & 1 \\
\hline & Jakucs et al. (2005) & P. alba & Hungary & 1 \\
\hline & Jakucs et al. (2005) & P. alba & Hungary & 1 \\
\hline & Jakucs et al. (2005) & Quercus cerris & Hungary & 1 \\
\hline
\end{tabular}


Jakucs et al. (2005)

Kaldorf (2004)

Massicotte et al. (1999b)

Hagerman et al. (2001)

Agerer (1996a)

Agerer (1996k)

Agerer \& Bougher (2001)

Raidl \& Müller (1996)

Raidl (1998b)

De Román \& De Miguel (2005)

Jakucs (2002a)

De Román \& De Miguel (2005)

Jakucs \& Agerer (1999b)

Jakucs (2002a)

Jakucs (2002h)

Jakucs et al. (2004)

Jakucs \& Agerer (2001)

Jakucs (2002a)

Jakucs (2002i)

Goodman (1996a)

Jakucs (2002a)

Agerer (1998e)

Ingleby et al. (1990)

Godbout \& Fortin (1985)

Agerer \& Waller (1993)

Duñabeitia et al. (1996)

Luppi \& Gautero (1967)

Uhl (1988a)

Comandini et al. (2004)

Abourouh \& Najim (1995)

Uhl (1988a)

Danielson (1984b)

Yamada et al. (2001)

Treu (1990a)

Lefevre \& Müller (1998)

Gill et al. (2000)

Danielson (1984b)

Yamada et al. (2001)

Yamada et al. (2001)

Cripps (1997b)

Brand (1992f)

Brand (19911)

Agerer (1987b)

Agerer (1987i)

Comandini et al. (2004)

Agerer (1987b)
Q. robur

Populus tremula $\times$ P. tremuloides

Hungary

Abies grandis, Arbutus menziesï,

USA

Lithocarpus densiflora, Pinus ponderosa

Canada

P. menziesii

Pinus sylvestris

P. sylvestris

Eucalyptus

Germany

Germany

Fagus sylvatica

F. sylvatica

Quercus ilex

Populus alba

Quercus ilex

Populus alba

P. alba

P. alba

Picea abies

Populus alba

P. alba

P. alba

Pseudotsuga menziesii

Populus alba

Pinus sylvestris

Picea sitchensis

Populus tremuloides

Fagus sylvatica

Pinus radiata

Quercus petraea

Abies alba

Cedrus atlantica

Betula pendula

Pinus banksiana

P. densiflora

Larix decidua

Pinus contorta

P. densiflora

P. banksiana

P. densiflora

P. densiflora

Populus tremuloides

Fagus sylvatica

Australia

Germany

Germany

Spain

Hungary

Spain

Hungary

Hungary

Hungary

Germany

Hungary

Hungary

Hungary

Canada

Hungary

UK

Canada

Germany

Spain

Italy

Germany

Italy

Morocco

Canada

Japan

Germany

Germany

Japan

Canada

Japan

Japan

USA

Germany

Germany

Germany

Picea abies

P. abies

Castanea sativa

Germany

Italy 
Table 1. (Cont.)

Ectomycorrhiza

Reference

Host tree

Country

Habitat

Tricholoma vaccinum (cont.)

Agerer (1987j)

Brunner et al. (1992)

Tricholomataceae

Truncocolumella citrina

See et al. (1997)

Eberhart \& Luoma (1996)

Hagerman et al. (2001)

Massicotte et al. (2000)

Tsugaerhiza luteoannulata

Agerer \& Molina (1997)

Agerer (1998f)

Agerer \& Trappe (1996)

Agerer (1998g)

Bradbury (1998)

Buée $e t$ al. (2004)

Chu-Chou \& Grace (1983)

Danielson \& Pruden (1989)

De Román \& De Miguel (2005)

Ingleby et al. (1990)

Jones et al. (1997)

Kaldorf (2004)

Kaldorf (2004)

Torres \& Honrubia (1997)

Ursic \& Peterson (1997)

T. aestivum

Bencivenga et al. (1992)

Etayo \& De Miguel (1998)

Giraud (1988)

Granetti (1995)

Granetti et al. (1995b)

Granetti et al. (1995b)

Granetti et al. (1995b)

Granetti et al. (1995b)

Granetti et al. (1995b)

Granetti et al. (1995b)

Granetti, Rubini \& Angelini (1995b)

Meotto, Nosenzo \& Fontana (1995)

Müller et al. (1996a)

Palenzona (1969)

Rauscher et al. (1996b)

Sáez \& De Miguel (1995)

Zambonelli, Salomoni \& Pisi (1993)

Zambonelli, Salomoni \& Pisi (1995)

Bencivenga et al. (1992)

Etayo \& De Miguel (1998)

Fontana \& Centrella (1967)

\section{Picea abies}

P. abies

Shorea leprosula

Pseudotsuga menziesii

P. menziesii

P. menziesii

Tsuga heterophylla

T. heterophylla

T. heterophylla

T. heterophylla

Pinus contorta

Fagus sylvatica

Pseudotsuga menziesii

Picea pungens

Quercus ilex

Betula pendula

B. papyrifera, Pseudotsuga menziesii

Populus tremula $\times P$. tremuloides

$P$. tremula $\times P$. tremuloides

Pinus halepensis

Pinus strobus

Corylus avellana, Quercus pubescens

C. avellana

Not specified

Corylus avellana, C. colurna, Ostrya

carpinifolia, Pinus halepensis, P. nigra,

Germany

Switzerland

Malays

USA

Canada

USA

Germany

USA

Germany

Canada

N. Zealand

Canada

Spain

UK

Canada

Germany

Germany

Spain

Canada

Italy

Spain

France

Italy

1

$P$. pinea, Quercus cerris, $Q$. ilex,

Q. pubescens

Corylus avellana

C. colurna

Pinus laricio

$P$. pinea

Quercus cerris

Q. ilex

Q. pubescens

Q. robur

Corylus avellana

C. avellana

C. avellana

Not specified

Quercus pubescens

Pinus pinea

Corylus avellana, Quercus pubescens

Q. faginea, Q. ilex

Corylus avellana, Pinus pinea,

Quercus petraea

$\begin{array}{ll}\text { Italy } & 3 \\ \text { Italy } & 3 \\ \text { Italy } & 3 \\ \text { Italy } & 3 \\ \text { Italy } & 3 \\ \text { Italy } & 3 \\ \text { Italy } & 3 \\ \text { Italy } & 3 \\ \text { France } & 3 \\ \text { Italy } & 3 \\ \text { France } & 3 \\ \text { Spain } & 4 \\ \text { Italy } & 3 \\ \text { Italy } & 3 \\ \text { Italy } & 4 \\ \text { Spain } & 4 \\ \text { Italy } & 1\end{array}$


Scannerini \& Palenzona (1967)

Pinus strobus

Spain

Voiry (1981)

Fagus

Zambonelli et al. (1993)

Duñabeitia et al. (1996)

Giomaro et al. (2000)

Giraud (1988)

Quercus pubescens

Pinus radiata

Tilia platyphyllos

Granetti \& Baciarelli (1997)

Not specified

Granetti (1995)

Cistus incanus, Corylus avellana,

C. colurna, Ostrya carpinifolia,

Quercus cerris, $Q$. pubescens, Pinus pinea,

Populus alba, P. nigra, Salix caprea,

Granetti, Angelini \& Rubini (1995a)

Rauscher et al. (1996a)

Rauscher et al. (1996c)

Zambonelli et al. (1995)

Zambonelli, Iotti \& Govi (1998)

T. brumale

Azul \& Freitas (1999)

Chevalier (1973)

Chevalier (1973)

Chevalier (1973)

Chevalier (1973)

De Román \& De Miguel (2005)

Etayo \& De Miguel (1998)

Fischer et al. (2004)

Fontana \& Bonfante-Fasolo (1971)

Giraud (1988)

Granetti \& Baciarelli (1997)

Granetti (1995)

Meotto et al. (1995)

Palenzona (1969)

Palenzona, Chevalier \& Fontana (1972)

Sáez \& De Miguel (1995)

Zambonelli et al. (1993)

Zambonelli et al. (1995)

Massicotte et al. (1999b)

T. californicum

T. excavatum

T. himalayense

T. indicum
Giraud (1988)

Pirazzi (1998)

Sáez \& De Miguel (1995)

Comandini \& Pacioni (1997)

Comandini \& Pacioni (1997)

Zambonelli et al. (1997)

Zambonelli, Tibiletti \& Pisi (1997)
Tilia platyphyllos
T. platyphyllos

Corylus avellana

C. avellana

Pinus pinea

P. pinea

Quercus suber

Corylus avellana

Pinus halepensis

P. nigra

P. sylvestris

Quercus ilex

Corylus avellana, $Q$. faginea

Q. ilex

Pinus nigra

Not specified

Quercus ilex

Corylus avellana, Ostrya carpinifolia,

Quercus ilex, $Q$. pubescens

Q. robur

Corylus avellana

C. avellana, Pinus nigra, Quercus pubescens

Not specified

Quercus pubescens

Pinus pinea

Abies grandis, Lithocarpus densiflora,

Pinus ponderosa, Pseudotsuga menziesii

Not specified

Quercus pubescens

Not specified

Quercus pubescens

Q. pubescens

Pinus pinea

Quercus cerris 
Table 1. (Cont.)

\begin{tabular}{|c|c|c|c|c|}
\hline Ectomycorrhiza & Reference & Host tree & Country & Habitat \\
\hline \multirow[t]{4}{*}{ Tuber macrosporum } & Giovannetti \& Fontana (1981) & Betula & Italy & 1 \\
\hline & Granetti (1995) & Quercus cerris & Italy & 3 \\
\hline & Meotto et al. (1995) & Q. robur & Italy & 3 \\
\hline & Zambonelli et al. (1993) & Q.pubescens & Italy & 3 \\
\hline \multirow[t]{2}{*}{ T. maculatum } & Fassi \& De Vecchi (1962) & Pinus strobus & Italy & 3 \\
\hline & Zambonelli et al. (1999) & Ostrya carpinifolia & Italy & 3 \\
\hline \multirow[t]{9}{*}{ T. magnatum } & Angelini \& Granetti (1995) & Populus alba & Italy & 2 \\
\hline & Granetti (1995) & $\begin{array}{l}\text { Corylus avellana, } \text { C. colurna, Ostrya } \\
\text { carpinifolia, Quercus cerris, Q. pubescens, } \\
\text { Q. robur, Populus alba, P. nigra, } \\
\text { Salix alba, S. caprea }\end{array}$ & Italy & 3 \\
\hline & Granetti et al. (1995a) & Tilia platyphyllos & Italy & 3 \\
\hline & Mello et al. (2001) & Quercus robur & Italy & 3 \\
\hline & Meotto et al. (1995) & Q. robur & Italy & 3 \\
\hline & Palenzona \& Fontana (1978) & Q. pubescens & Italy & 3 \\
\hline & Rubini et al. (2001) & Q. cerris & Italy & 3 \\
\hline & Sáez \& De Miguel (1995) & Not specified & Spain & 4 \\
\hline & Zambonelli et al. (1993) & Quercus pubescens & Italy & 3 \\
\hline \multirow[t]{14}{*}{ T. melanosporum } & Águeda et al. (2001) & Q. ilex & Spain & 4 \\
\hline & Bencivenga et al. (1992) & Corylus avellana, $Q$. pubescens & Italy & 4 \\
\hline & Etayo \& De Miguel (1998) & C. avellana, $Q$. faginea, $Q$. ilex & Spain & 4 \\
\hline & Giraud (1988) & Not specified & France & 4 \\
\hline & Granetti \& Baciarelli (1997) & Quercus ilex & Italy & 4 \\
\hline & Granetti (1995) & $\begin{array}{l}\text { Cistus incanus, Corylus avellana, } \\
\text { C. colurna, Ostrya carpinifolia, Quercus } \\
\text { cerris, } Q . \text { ilex, } Q \text {. pubescens, } Q \text {. robur }\end{array}$ & Italy & 3 \\
\hline & Meotto et al. (1995) & O. robur & Italy & 3 \\
\hline & Palenzona (1969) & Corylus avellana & Italy & 3 \\
\hline & Palenzona et al. (1972) & $\begin{array}{l}\text { C. avellana, Pinus sylvestris, } \\
\text { Quercus pubescens }\end{array}$ & Italy & 3 \\
\hline & Rauscher \& Chevalier (1995a) & Corylus avellana & France & 3 \\
\hline & Rauscher, Agerer \& Chevalier (1995) & C. avellana & France & 3 \\
\hline & Sáez \& De Miguel (1995) & Not specified & Spain & 4 \\
\hline & Zambonelli et al. (1993) & Quercus pubescens & Italy & 3 \\
\hline & Zambonelli et al. (1995) & Pinus pinea & Italy & 3 \\
\hline \multirow[t]{8}{*}{ T. mesentericum } & Giraud (1988) & Not specified & France & 4 \\
\hline & Granetti (1995) & Corylus avellana & Italy & 3 \\
\hline & Meotto et al. (1995) & Ostrya carpinifolia & Italy & 3 \\
\hline & Rauscher \& Chevalier (1995b) & Corylus avellana & France & 3 \\
\hline & Rauscher et al. (1995) & C. avellana & France & 3 \\
\hline & Sáez \& De Miguel (1995) & Not specified & Spain & 4 \\
\hline & Zambonelli et al. (1993) & Quercus pubescens & Italy & 3 \\
\hline & Zambonelli et al. (1995) & Pinus pinea & Italy & 3 \\
\hline T. oligospermum & Bencivenga, Tanfulli \& Donnini (1997) & Quercus cerris & Italy & 3 \\
\hline
\end{tabular}


Jakucs (2002a)

Giraud (1988)

Palenzona et al. (1972)

\section{Pirazzi (1998)}

Rauscher \& Chevalier (1995c)

Rauscher et al. (1995)

Sáez \& De Miguel (1995)

Müller et al. (1996b)

See et al. (1997)

Kernaghan (2001)

Uhl (1989)

Eberhardt, Walter \& Kottke (1999)

Tylopilus felle

Tylopilus felleus
Tylospora asterophora

T. fibrillosa

Unidentified

Eberhardt et al. (1999)

Taylor \& Alexander (1991)

Abourouh \& Najim (1995)

Abourouh \& Najim (1995)

Abourouh \& Najim (1995)

Abourouh \& Najim (1995)

Abourouh \& Najim (1995)

Abourouh \& Najim (1995)

Abourouh \& Najim (1995)

Abourouh \& Najim (1995)

Abourouh \& Najim (1995)

Abourouh \& Najim (1995)

Abourouh \& Najim (1995)

Abourouh \& Najim (1995)

Águeda et al. (2001)

Agueda et al. (2001)

Águeda et al. (2001)

Agueda et al. (2001)

Águeda et al. (2001)

Águeda et al. (2001)

Azul \& Freitas (1999)

Azul \& Freitas (1999)

Baxter et al. (1999)

Bencivenga et al. (1992)

Bencivenga et al. (1992)

Bencivenga et al. (1992)

Bencivenga et al. (1995)

Bencivenga et al. (1995)

Buée et al. (2004)

Buée et al. (2004)

Buée et al. (2004)

Populus alba

Not specified

Abies alba, Carpinus betulus, Corylus

P. nigra, P. strobus, P. sylvestris,

Quercus pubescens, Tilia platyphyllos

Q. pubescens

Corylus avellana

C. avellana

Not specified

Corylus avellana

Shorea leprosula

Picea engelmannit

Pinus sylvestris

Picea abies

P. abies

P. sitchensis

Cedrus atlantica

C. atlantica

C. atlantica

C. atlantica

C. atlantica

C. atlantica

C. atlantica

C. atlantica

C. atlantica

C. atlantica

C. atlantica

C. atlantica

Quercus ilex

Q. ilex

Q. ilex

Q. ilex

Q. ilex

Q. ilex

Q. suber

Q. suber

Quercus

Corylus avellana, Quercus pubescens

C. avellana, $Q$. pubescen

C. avellana, $Q$. pubescen

Not specified

Not specified

Fagus sylvatica

F. sylvatica

F. sylvatica
Germany

Hungary

France

Italy

France

France

Spain

France

Malaysia

Canada

Germany

Germany

Germany

UK

Morocco

Morocco

Morocco

Morocco

Morocco

Morocco

Morocco

Morocco

Morocco

Morocco

Morocco

Morocco

Spain

Spain

Spain

Spain

Spain
Spain

Portugal

Portugal

USA

Italy

Italy

Italy

Italy

France

France

France 
Table 1. (Cont.)

\begin{tabular}{|c|c|c|c|c|}
\hline Ectomycorrhiza & Reference & Host tree & Country & Habitat \\
\hline \multirow[t]{42}{*}{ Unidentified (cont.) } & Buée et al. (2004) & Fagus sylvatica & France & 1 \\
\hline & Chu-Chou \& Grace (1983) & Pseudotsuga menziesii & N. Zealand & 1 \\
\hline & Chu-Chou \& Grace (1983) & P. menziesii & N. Zealand & 1 \\
\hline & Chu-Chou \& Grace (1983) & P. menziesii & N. Zealand & 1 \\
\hline & Chu-Chou \& Grace (1983) & P. menziesii & N. Zealand & 1 \\
\hline & De Román \& De Miguel (2005) & Quercus ilex & Spain & 1 \\
\hline & De Román \& De Miguel (2005) & Q. ilex & Spain & 1 \\
\hline & De Román \& De Miguel (2005) & Q. ilex & Spain & 1 \\
\hline & De Román \& De Miguel (2005) & Q. ilex & Spain & 1 \\
\hline & De Román \& De Miguel (2005) & Q. ilex & Spain & 1 \\
\hline & De Román \& De Miguel (2005) & Q. ilex & Spain & 1 \\
\hline & De Román \& De Miguel (2005) & Q. ilex & Spain & 1 \\
\hline & De Román \& De Miguel (2005) & Q. ilex & Spain & 1 \\
\hline & De Román \& De Miguel (2005) & Q. ilex & Spain & 1 \\
\hline & De Román \& De Miguel (2005) & Q. ilex & Spain & 1 \\
\hline & De Román \& De Miguel (2005) & Q. ilex & Spain & 1 \\
\hline & De Román \& De Miguel (2005) & Q. ilex & Spain & 1 \\
\hline & De Román \& De Miguel (2005) & Q. ilex & Spain & 1 \\
\hline & De Román \& De Miguel (2005) & Q. ilex & Spain & 1 \\
\hline & De Román \& De Miguel (2005) & Q. ilex & Spain & 1 \\
\hline & De Román \& De Miguel (2005) & Q. ilex & Spain & 1 \\
\hline & Donnini \& Bencivenga (1995) & Corvlus avellana & Italy & 4 \\
\hline & Donnini \& Bencivenga (1995) & C. avellana & Italy & 4 \\
\hline & Donnini \& Bencivenga (1995) & C. avellana & Italy & 4 \\
\hline & Donnini \& Bencivenga (1995) & C. avellana & Italy & 4 \\
\hline & Donnini \& Bencivenga (1995) & C. avellana & Italy & 4 \\
\hline & Donnini \& Bencivenga (1995) & $\begin{array}{l}\text { C. avellana, Ostrya carpinifolia, } \\
\text { Populus alba, Salix caprea }\end{array}$ & Italy & 4 \\
\hline & Donnini \& Bencivenga (1995) & $\begin{array}{l}\text { Corylus avellana, Quercus pubescens, } \\
\text { Salix caprea }\end{array}$ & Italy & 4 \\
\hline & Donnini \& Bencivenga (1995) & Populus alba, Quercus pubescens & Italy & 4 \\
\hline & Donnini \& Bencivenga (1995) & Quercus & Italy & 4 \\
\hline & Donnini \& Bencivenga (1995) & Quercus & Italy & 4 \\
\hline & Donnini \& Bencivenga (1995) & Q. pubescens & Italy & 4 \\
\hline & Donnini \& Bencivenga (1995) & Q. pubescens & Italy & 4 \\
\hline & Donnini \& Bencivenga (1995) & Quercus, Salix & Italy & 4 \\
\hline & Fontana (1961) & Populus & Italy & 1 \\
\hline & Fontana (1961) & Populus & Italy & 1 \\
\hline & Fontana (1961) & Populus & Italy & 1 \\
\hline & Fontana (1961) & Populus & Italy & 1 \\
\hline & Fontana (1961) & Populus & Italy & 1 \\
\hline & Fontana (1961) & Populus & Italy & 1 \\
\hline & Fontana (1961) & Populus & Italy & 1 \\
\hline & Fontana (1961) & Populus & Italy & 1 \\
\hline
\end{tabular}


Granetti \& Baciarelli (1997)

Granetti \& Baciarelli (1997)

Granetti \& Baciarelli (1997)

Hagerman et al. (2001)

Ingleby et al. (1990)

Ingleby et al. (1990)

Ingleby et al. (1990)

Ingleby et al. (1990)

Jones et al. (1997)

Jones et al. (1997)

Jones et al. (1997)

Jones et al. (1997)

Jones et al. (1997)

Jones et al. (1997)

Jones et al. (1997)

Jones et al. (1997)

Jones et al. (1997)

Jones et al. (1997)

Jones et al. (1997)

Jones et al. (1997)

Jones et al. (1997)

Jones et al. (1997)

Jones et al. (1997)

Jones et al. (1997)

Jones et al. (1997)

Jones et al. (1997)

Kaldorf (2004)

Kaldorf (2004)

Kaldorf (2004)

Kaldorf (2004)

Kaldorf (2004)

Kaldorf (2004)

Kaldorf (2004)

T. heterophylla

Corylus avellana, Ostrya carpinifolia,

Quercus ilex, $Q$. pubescens

Ostrya carpinifolia

Quercus ilex

Q. ilex

Q. ilex

Q. ilex

Q. ilex

Pseudotsuga menziesii

Picea sitchensis

P. sitchensis

P. sitchensis

$P$. sitchensis

Betula papyrifera, Pseudotsuga menziesii

B. papyrifera

B. papyrifera

B. papyrifera, Pseudotsuga menziesii

B. papyrifera, $P$. menziesii

$B$. papyrifera, $P$. menziesii

B. papyrifera, $P$. menziesii

B. papyrifera, $P$. menziesii

B. papyrifera, P. menziesii

$B$. papyrifera, $P$. menziesii

B. papyrifera, $P$. menziesii

B. papyrifera, $P$. menziesii

P. menziesii

P. menziesii

P. menziesi

P. menziesil

P. menziesil

P. menziesii

Populus tremula $\times P$. tremuloides

$P$. tremula $\times P$. tremuloides

$P$. tremula $\times P$. tremuloides

$P$. tremula $\times P$. tremuloides

$P$. tremula $\times P$. tremuloides

$P$. tremula $\times P$. tremuloides

Canada

Canada

Canada

Canada

Canada

Canada

Canada

Canada

Germany

Germany

Germany

Germany

Germany

Germany

$P$. tremula $\times P$. tremuloides 
Table 1. (Cont.)

\begin{tabular}{|c|c|c|c|c|}
\hline Ectomycorrhiza & Reference & Host tree & Country & Habitat \\
\hline \multirow[t]{43}{*}{ Unidentified (cont.) } & Kaldorf (2004) & Populus tremula $\times P$. tremuloides & Germany & 1 \\
\hline & Massicotte et al. (1999b) & $\begin{array}{l}\text { Abies grandis, Lithocarpus densiflora, } \\
\text { Pseudotsuga menziesii }\end{array}$ & USA & 3 \\
\hline & Miller et al. (1990) & Alnus rubra & USA & 5 \\
\hline & Miller et al. (1990) & A. rubra & USA & 5 \\
\hline & Miller et al. (1990) & A. rubra & USA & 5 \\
\hline & Miller et al. (1990) & A. rubra & USA & 5 \\
\hline & Moser et al. (2005) & Quercus garryana & USA & 1 \\
\hline & Moser et al. (2005) & Q. garryana & USA & 1 \\
\hline & Moser et al. (2005) & Q. garryana & USA & 1 \\
\hline & Moser et al. (2005) & Q. garryana & USA & 1 \\
\hline & Moser et al. (2005) & Q. garryana & USA & 1 \\
\hline & Moser et al. (2005) & Q. garryana & USA & 1 \\
\hline & Moser et al. (2005) & Q. garryana & USA & 1 \\
\hline & Moser et al. (2005) & Q. garryana & USA & 1 \\
\hline & Moser et al. (2005) & Q. garryana & USA & 1 \\
\hline & Moser et al. (2005) & Q. garryana & USA & 1 \\
\hline & Moser et al. (2005) & Q. garryana & USA & 1 \\
\hline & Moser et al. (2005) & Q. garryana & USA & 1 \\
\hline & Moser et al. (2005) & Q. garryana & USA & 1 \\
\hline & Moser et al. (2005) & Q. garryana & USA & 1 \\
\hline & Moser et al. (2005) & Q. garryana & USA & 1 \\
\hline & Moser et al. (2005) & Q. garryana & USA & 1 \\
\hline & Moser et al. (2005) & Q. garryana & USA & 1 \\
\hline & Moser et al. (2005) & Q. garryana & USA & 1 \\
\hline & Moser et al. (2005) & Q. garryana & USA & 1 \\
\hline & Moser et al. (2005) & Q. garryana & USA & 1 \\
\hline & Moser et al. (2005) & Q. garryana & USA & 1 \\
\hline & Moser et al. (2005) & Q. garryana & USA & 1 \\
\hline & Moser et al. (2005) & Q. garryana & USA & 1 \\
\hline & Moser et al. (2005) & Q. garryana & USA & 1 \\
\hline & Moser et al. (2005) & Q. garryana & USA & 1 \\
\hline & Moser et al. (2005) & Q. garryana & USA & 1 \\
\hline & Moser et al. (2005) & Q. garryana & USA & 1 \\
\hline & Moser et al. (2005) & Q. garryana & USA & 1 \\
\hline & Thomas \& Jackson (1979) & Picea sitchensis & UK & 3 \\
\hline & Thomas \& Jackson (1979) & P. sitchensis & UK & 3 \\
\hline & Thomas \& Jackson (1979) & P. sitchensis & UK & 3 \\
\hline & Thomas \& Jackson (1979) & P. sitchensis & UK & 3 \\
\hline & Thomas \& Jackson (1979) & P. sitchensis & UK & 3 \\
\hline & Torres \& Honrubia (1997) & Pinus halepensis & Spain & 3 \\
\hline & Treu (1990a) & Larix decidua & Germany & 1 \\
\hline & Trost et al. (1999) & Picea abies & Slovenia & 1 \\
\hline & Trost et al. (1999) & P. abies & Slovenia & 1 \\
\hline
\end{tabular}


Voiry (1981)

Voiry (1981)

Voiry (1981)

Wöllecke (2001)

Fagus

Fagus

Fagus

Wöllecke (2001)

Wöllecke (2001)

Wöllecke (2001)

Wöllecke (2001)

Wöllecke (2001)

Wöllecke (2001)

Wöllecke (2001)

Wöllecke (2001)

Wöllecke (2001)

Wöllecke (2001)

Zambonelli et al. (1998)

Zambonelli et al. (1998)

Zambonelli et al. (1998)

Zambonelli et al. (1998)

Zambonelli et al. (1998)

Xerocomus

$X$. armeniacus

$X$. badius

Torres \& Honrubia (1997)

Jakucs (2002a)

Agerer \& Gronbach (1990b)

Duñabeitia et al. (1996)

Gronbach (1988)

Wöllecke (2001)

X. chrysenteron

Azul \& Freitas (1999)

Brand (1989a)

Brand (1989b)

Jakucs \& Beenken (2001)

Jakucs (2002j)

Palfner (1995b)

X. subtomentosus
Slovenia

Slovenia

Slovenia

Slovenia

Slovenia

Canada

France

France

France

France

France

Germany

Germany

Germany

Germany

Germany

Germany

Germany

Germany

Germany

Germany

Germany

Italy

Italy

Italy

Italy

Italy

Spain

Hungary

Germany

Spain

Germany

Germany

Portugal

Germany

Germany

Hungary

Hungary

Germany

F. sylvatica

Q. cerris

Q. robur 
Numerous descriptions of ECM have also been published in different journals following any of the systems mentioned above, but the information on the topic is typically difficult to approach due to the varied nature of the sources. Therefore, we considered it necessary to gather and review these published sources in order to build a database of the descriptions of ECM published so far useful to all mycorrhizologists. We have focused exclusively on morphological descriptions of ECM. It is outside the scope of this work to refer to other types of mycorrhizas (e.g. ectendomycorrhizas, ericoid, or arbutoid mycorrhizas) or to ECM morphotypes cited in papers dealing with molecular techniques which do not provide a morphological description. Although this revision is very extensive, some sources could not be accessed, and there may be other descriptions hidden as grey literature or in journals with little circulation. Contributions by other authors are thus expected and welcome in order to enrich the database.

\section{MATERIALS AND METHODS}

Each published source containing a description of an ECM, no matter how brief the description, was reviewed to record the following details: (1) fungus forming the ECM; (2) host tree; (3) country where the material for description was collected; and (4) habitat of the ECM, i.e. whether the ECM was collected from the field, a nursery, a truffle growing stand, or if it was synthesized in vitro. Only published sources were reviewed, i.e. ECM descriptions contained in theses, reports, or other kinds of unpublished material were not considered. ECM from nurseries included artificially inoculated or naturally occurring in the nursery. When an ECM morphotype was collected in more than one habitat, the term 'various' was applied. For simplicity, subspecies and varieties of fungi and host trees were not considered. In some cases, several host tree species were reported to be associated with the same ECM morphotype in the same description, but no morphological or anatomical distinction was made between ECM tips from the different tree species. In these cases, all host tree species were cited in Table 1 within the same entry. The term 'not specified' was used when several host tree species were mentioned in a paper, but there was no specific information about the tree species actually associated with each ECM described.

Other secondary data were also recorded: the division, order, sporocarp morphology and sporocarp development (epigeous or hypogeous) of the fungus forming the ECM; whether the description was detailed (Agerer 1986a, 1987-2002, 1994a, 1999a); and whether drawings and photos were also available together with the description. The categories used for fruit body morphology were: gasteroid, mushroom (both agaricoid and boletoid), truffle (any hypogeous fungus, including Ascomycota and Basidiomycota), and other morphology (apothecial, perithecial, clavarioid,

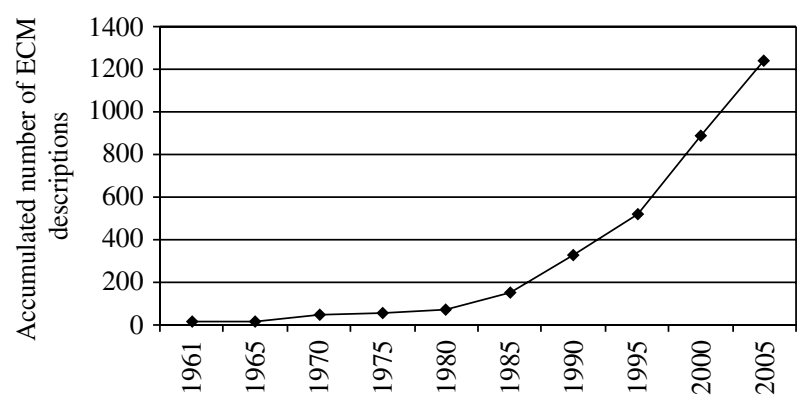

Fig. 1. Accumulated number of ECM descriptions published since 1961.

ramarioid or resupinate). Although some comments are made in this review regarding these secondary data, the data are not included in full due to space constraints, but the corresponding author will provide them if contacted.

\section{RESULTS AND DISCUSSION}

In all 1244 descriptions of ECM published in 479 papers were reviewed (Table 1). The number of different ECM morphotypes described was 814, with each ECM not identified to species level considered a different ECM. 345 ECM morphotypes were identified to species, 132 to genus, 21 to family, five to order, and 20 to division; four had another level of identification (cortinarioid, pezizalean, sebacinoid, and tomentelloid, respectively), 99 were unidentified ECM morphotypes published under a binomial name (Agerer 1986a, 1987-2002, 1994a, 1999a) or other name widely used in the literature (morphotypes AD and SB; Giraud 1988), and 188 were unidentified ECM morphotypes lacking a name. Only 330 of the 1244 descriptions reviewed were detailed (Agerer 1986a, 1987-2002, 1994a, 1999a); 379 descriptions included drawings and 771 provided photographs.

The descriptions reviewed date back to 1961, and include all papers published until May 2005, when the revision of this article was submitted. Fig. 1 shows the accumulated number of ECM descriptions published over the years, and it is important to note the sharp increase in publications from 1985 onwards, when Agerer (1986a) started to develop the more defined system.

Most of the ECM described were collected in Europe, with 862 descriptions recorded as follows: Austria, 2; Belgium, 1; Estonia, 3; France, 83; Germany, 331; Hungary, 46; Italy, 177; Poland, 28; Portugal, 14; Slovenia, 15; Spain, 93; Sweden, 2; Switzerland, 14; UK, 53. A significant number, 271 ECM, were collected in North America (Canada, 175; USA, 96). All other continents remain poorly represented: 37 ECM were collected in South America (Argentina, 3; Chile, 28; Ecuador, 6), 21 in Africa (Cameroon, 7; Morocco, 14), 37 in Asia (India, 2; Indonesia, 1; Japan, 14; 
Malaysia, 10; Taiwan, 10) and 16 in Oceania (Australia, 2; New Zealand, 14). It is interesting to note that most descriptions came from Germany, Italy, and Canada. The material collected in Germany was usually described in detail following the system developed by Agerer (1986a, 1987-2002, 1994a, 1999a), and is the most extensive source of information on morphology and anatomy of ECM. In contrast, the ECM described in Canada were usually collected in ecological studies of ectomycorrhizal communities, and the descriptions provided were typically short, with the exception of those published by Goodman et al. (1996-2000). In Italy, research on ECM is often linked to the interest in cultivating truffles, and most ECM described were either Tuber species of commercial interest or competitive fungi that threaten truffle production.

Gymnosperms were the most common tree associates, recorded in 510 descriptions (Abies, 15; Cedrus, 14; Gnetum, 1; Larix, 20; Picea, 162; Pinus, 226; Pseudotsuga, 59; Tsuga, 13). Genera in the Fagaceae were also well represented, with 339 descriptions (Castanea, 3; Castanopsis, 1; Cyclobalanopsis, 1; Fagus, 116; Nothofagus, 29; Pasania, 1; Quercus, 188). Other families recorded as hosts were: Betulaceae, 131 (Alnus, 50; Betula, 48; Carpinus, 4; Corylus, 26; Ostrya, 3); Salicaceae, 94 (Populus, 87; Salix, 7); Cistaceae, 18 (Cistus, 3 ; Fumana, 4 ; Helianthemum, 11); Myrtaceae, 7 (Eucalyptus, 5; Kunzea, 1; Leptospermum, 1); Leguminosae, 7 (Acacia, 1; Tetraberlinia, 6); Nyctaginaceae, 6 (Guapira, 1; Neea, 5); Dipterocarpaceae, 11 (Shorea); Rosaceae, 1 (Dryas); Tiliaceae, 6 (Tilia). The remaining 113 ECM were associated with either 'not specified' host trees (35), or with more than one host tree species, within the same description (78). If the ECM description applied to more than one host tree species, the records were not considered individually for the count to avoid an artificial increase in the total number of descriptions. A careful analysis of the host species involved in the ECM described revealed that boreal and temperate forests are the best-known ecosystems so far, whereas there is little information on ECM communities of tropical and Mediterranean ecosystems.

Most ECM were collected from the field (899), 159 in a nursery, 81 in a truffle growing stand. Sixty five descriptions were based in ECM material synthesized in vitro, and in 40 descriptions, the material was collected in more than one habitat (indicated by the term 'various'). Many ECM morphotypes collected from the field could not be identified, whereas the aim of inoculating ECM fungi in nurseries or synthesizing them in vitro was precisely to know the identity of the fungal symbiont beforehand. Fungi recorded in truffle growing stands were typically truffle species or their competitors.

The associated fungi recorded in the 1244 ECM descriptions reviewed were mostly Basidiomycota (707), 200 were Ascomycota, and in 337 descriptions the fungal division could not be ascertained. As most Ascomycota ECM were Tuber species, it is clear that there is a lack of knowledge on the ECM of this fungal division. Fungal orders were represented in ECM descriptions as follows: Agaricales, 220; Boletales, 166; Cantharellales, 13; Elaphomycetales, 5; Hymenochaetales, 1; Pezizales, 150; Phallales, 18; Polyporales, 41; Russulales, 148; Thelephorales, 77; Tremellales, 5; unidentified order, 400.

The majority of fungal species cited were epigeous (638), with only 198 descriptions belonging to hypogeous fungi. The type of fruit body development of the fungal symbiont was unknown in the remaining 408 descriptions. These data show that, with the exception of Tuber species again, the ECM of hypogeous fungi are poorly known. The fact that fruit bodies of hypogeous fungi are typically difficult to find is definitely related to the scarce interest researchers have hitherto shown for ECM formed by these species.

The fruit body morphology of the associated fungus was mushroom-like in 460 ECM descriptions, gasteroid in 35, truffle-like in 198, had other morphologies in 142, and could not be assigned to any morphology in 409 descriptions. These data indicated that fungi which lack the usual 'mushroom' morphology or which have inconspicuous fruit bodies have been traditionally overlooked in ECM community studies (Gardes \& Bruns 1996, Dahlberg, Jonsson \& Nylund 1997, Stendell, Horton \& Bruns 1999, Taylor \& Bruns 1999, Peter et al. 2001).

The amount of research carried out so far on description and identification of fungi involved in ECM communities is outstanding. Nevertheless, this review reveals that many gaps in knowledge remain to be filled, and mycorrhizologists should focus on littleknown geographic areas, ecosystems, host trees and fungal groups in future studies in order to face the challenges the ECM community poses.

\section{ACKNOWLEDGMENTS}

We are grateful to Steve Woodward and two anonymous referees for their useful comments on the manuscript. This revision was carried out over many years, and there are several funding bodies we wish to thank for their financial support: the Spanish Instituto Nacional de Investigaciones Agrarias y Alimentarias for a PhD grant for M. de R.; Gobierno de Navarra and Fundación Universitaria de Navarra for funding three research projects; and the Spanish Ministerio de Educación y Ciencia for a post-doctoral grant for M. de R.

\section{REFERENCES}

Abourouh, M. \& Najim, L. (1995) Les différents types d'ectomycorhizes naturelles de Cedrus atlantica Manetti au Maroc. Cryptogamic Botany 5: 332-340.

Agerer, R. (1986a) Studies on ectomycorrhizae II. Introducing remarks on characterization and identification. Mycotaxon 26: 473-492. 
Agerer, R. (1986b) Studies on ectomycorrhizae III. Mycorrhizae formed by four fungi in the genera Lactarius and Russula on spruce. Mycotaxon 27: 1-59.

Agerer, R. (1987-2002) Colour Atlas of Ectomycorrhizae. EinhornVerlag, Munich.

Agerer, R. (1987a) Studies on ectomycorrhizae V. Mycorrhizae formed by Dermocybe cinnamomea and D. sanguinea on spruce. Nova Hedwigia 44: 69-89.

Agerer, R. (1987b) Studies on ectomycorrhizae IX. Mycorrhizae formed by Tricholoma sulphureum and T. vaccinum on spruce. Mycotaxon 28: 327-360.

Agerer, R. (1987c) Studies on ectomycorrhizae X. Mycorrhizae formed by Cortinarius obtusus and C. venetus on spruce. Mycologia 79: 524-539.

Agerer, R. (1987d) Dermocybe sanguinea. In Colour Atlas of Ectomycorrhizae (R. Agerer, ed.): pl. 8. Einhorn Verlag, Schwäbisch Gmünd.

Agerer, R. (1987e) Lactarius deterrimus. In Colour Atlas of Ectomycorrhizae (R. Agerer, ed.): pl. 3, Einhorn Verlag, Schwäbisch Gmünd.

Agerer, R. (1987f) Lactarius picinus. In Colour Atlas of Ectomycorrhizae (R. Agerer, ed.): pl. 4. Einhorn Verlag, Schwäbisch Gmünd.

Agerer, R. (1987g) Russula ochroleuca. In Colour Atlas of Ectomycorrhizae (R. Agerer, ed.): pl. 1. Einhorn Verlag, Schwäbisch Gmünd.

Agerer, R. (1987h) Russula xerampelina. In Colour Atlas of Ectomycorrhizae (R. Agerer, ed.): pl. 2. Einhorn Verlag, Schwäbisch Gmünd.

Agerer, R. (1987i) Tricholoma sulphureum. In Colour Atlas of Ectomycorrhizae (R. Agerer, ed.): pl. 9. Einhorn Verlag, Schwäbisch Gmünd.

Agerer, R. (1987j) Tricholoma vaccinum. In Colour Atlas of Ectomycorrhizae (R. Agerer, ed.): pl. 10. Einhorn Verlag, Schwäbisch Gmünd.

Agerer, R. (1988a) Cortinarius venetus. In Colour Atlas of Ectomycorrhizae (R. Agerer, ed.): pl. 13. Einhorn Verlag, Schwäbisch Gmünd.

Agerer, R. (1988b) Studies on ectomycorrhizae XIV. Mycorrhizae formed by Cortinarius hercynicus and C. variecolor on Picea abies. Canadian Journal of Botany 66: 2068-2078.

Agerer, R. (1989) Cortinarius variecolor. In Colour Atlas of Ectomycorrhizae (R. Agerer, ed.): pl. 24. Einhorn Verlag, Schwäbisch Gmünd.

Agerer, R. (1990a) Studies on ectomycorrhizae XXIV. Ectomycorrhizae of Chroogomphus helveticus and C. rutilus (Gomphidiaceae, Basidiomycetes) and their relationship to those of Suillus and Rhizopogon. Nova Hedwigia 50: 1-63.

Agerer, R. (1990b) Chroogomphus helveticus ssp. tatrensis. In Colour Atlas of Ectomycorrhizae (R. Agerer, ed.): pl. 37. Einhorn Verlag, Schwäbisch Gmünd.

Agerer, R. (1990c) Cortinarius hercynicus. In Colour Atlas of Ectomycorrhizae (R. Agerer, ed.): pl. 38. Einhorn Verlag, Schwäbisch Gmünd.

Agerer, R. (1991a) Studies on ectomycorrhizae XXXIV. Mycorrhizae of Gomphidius glutinosus and of G. roseus with some remarks on Gomphidiaceae (Basidiomycetes). Nova Hedwigia 53: 127-170.

Agerer, R. (1991b) Gomphidius glutinosus. In Colour Atlas of Ectomycorrhizae (R. Agerer, ed.): pl. 58. Einhorn Verlag, Schwäbisch Gmünd.

Agerer, R. (1991c) Sarcodon imbricatus. In Colour Atlas of Ectomycorrhizae (R. Agerer, ed.): pl. 66. Einhorn Verlag, Schwäbisch Gmünd.

Agerer, R. (1992a) Studies on ectomycorrhizae XLIV. Ectomycorrhizae of Boletopsis leucomelaena (Thelephoraceae, Basidiomycetes) and their relationship to an unidentified ectomycorrhiza. Nova Hedwigia 55: 501-518.

Agerer, R. (1992b) Gomphidius roseus. In Colour Atlas of Ectomycorrhizae (R. Agerer, ed.): pl. 71. Einhorn Verlag, Schwäbisch Gmünd.

Agerer, R. (1993a) Boletopsis leucomelaena. In Colour Atlas of Ectomycorrhizae (R. Agerer, ed.): pl. 75. Einhorn Verlag, Schwäbisch Gmünd.
Agerer, R. (1993b) Hydnellum peckii. In Colour Atlas of Ectomycorrhizae (R. Agerer, ed.): pl. 77. Einhorn Verlag, Schwäbisch Gmünd.

Agerer, R. (1993c) Phellodon niger. In Colour Atlas of Ectomycorrhizae (R. Agerer, ed.): pl. 78. Einhorn Verlag, Schwäbisch Gmünd.

Agerer, R. (1993d) Ectomycorrhizae of Hydnellum peckii on Norway spruce and their chlamydospores. Mycologia 8 (1): 74-83.

Agerer, R. (1994a) Characterization of ectomycorrhiza. In Techniques for Mycorrhizal Research, (J. R. Norris, D. Read \& A. K. Varma eds): 25-73. Academic Press, London.

Agerer, R. (1994b) Pseudotomentella tristis. In Colour Atlas of Ectomycorrhizae (R. Agerer, ed.): pl. 84. Einhorn Verlag, Schwäbisch Gmünd.

Agerer, R. (1996a) Ectomycorrhizae of Tomentella albomarginata (Thelephoraceae) on Scots pine. Mycorrhiza 6: 1-7.

Agerer, R. (1996b) Albatrellus ovinus (Schaeff.: Fr.) Kotl. \& Pouz. + Picea abies (L) Karst. Descriptions of Ectomycorrhizae 1: 23-28.

Agerer, R. (1996c) Ramaria aurea (Schaeff.: Fr.) Quél.+Fagus sylvatica L. Descriptions of Ectomycorrhizae 1: 107-112.

Agerer, R. (1996d) Ramaria largentii Marr \& D.E. Stuntz+ Picea abies (L.) H. Karst. Descriptions of Ectomycorrhizae 1: 113-118.

Agerer, R. (1996e) Ramaria spinulosa (Fr.) Quél. + Fagus sylvatica L. Descriptions of Ectomycorrhizae 1: 119-124.

Agerer, R. (1996f) Ramaria subbotrytis (Coker) Corner + Quercus robur L. Descriptions of Ectomycorrhizae 1: 125-130.

Agerer, R. (1996g) Albatrellus ovinus. In Colour Atlas of Ectomycorrhizae (R. Agerer, ed.): pl. 91. Einhorn Verlag, Schwäbisch Gmünd.

Agerer, R. (1996h) Ramaria largentii. In Colour Atlas of Ectomycorrhizae (R. Agerer, ed.): pl. 103. Einhorn Verlag, Schwäbisch Gmünd.

Agerer, R. (1996i) Rhizopogon subcaerulescens. In Colour Atlas of Ectomycorrhizae (R. Agerer, ed.): pl. 104. Einhorn Verlag, Schwäbisch Gmünd.

Agerer, R. (1996j) Russula acrifolia. In Colour Atlas of Ectomycorrhizae (R. Agerer, ed.): pl. 105. Einhorn Verlag, Schwäbisch Gmünd.

Agerer, R. (1996k) Tomentella albomarginata. In Colour Atlas of Ectomycorrhizae (R. Agerer, ed.): pl. 111. Einhorn Verlag, Schwäbisch Gmünd.

Agerer, R. (1997) Entoloma sinuatum (Bull.: Fr.) Kummer + Salix spec. Descriptions of Ectomycorrhizae 2: 13-18.

Agerer, R. (1998a) Entoloma sinuatum. In Colour Atlas of Ectomycorrhizae (R. Agerer, ed.): pl. 117. Einhorn Verlag, Schwäbisch Gmünd.

Agerer, R. (1998b) Geastrum fimbriatum. In Colour Atlas of Ectomycorrhizae (R. Agerer, ed.): pl. 119. Einhorn Verlag, Schwäbisch Gmünd.

Agerer, R. (1998c) Lyophyllum decastes. In Colour Atlas of Ectomycorrhizae (R. Agerer, ed.): pl. 124. Einhorn Verlag, Schwäbisch Gmünd.

Agerer, R. (1998d) Ramaria subbotrytis. In Colour Atlas of Ectomycorrhizae (R. Agerer, ed.): pl. 134. Einhorn Verlag, Schwäbisch Gmünd.

Agerer, R. (1998e) Tomentellopsis submollis. In Colour Atlas of Ectomycorrhizae (R. Agerer, ed.): pl. 138. Einhorn Verlag, Schwäbisch Gmünd.

Agerer, R. (1998f) Tsugaerhiza luteoannulata. In Colour Atlas of Ectomycorrhizae (R. Agerer, ed.): pl. 139. Einhorn Verlag, Schwäbisch Gmünd.

Agerer, R. (1998g) Tsugaerhiza tripigmentata. In Colour Atlas of Ectomycorrhizae (R. Agerer, ed.): pl. 140. Einhorn Verlag, Schwäbisch Gmünd.

Agerer, R. (1999a) Anatomical characteristics of identified ectomycorrhizas: an attempt towards a natural classification. In: Mycorrhiza: structure, function, molecular biology and biotechnology (A. Varma \& B. Hock, eds): 633-682. 2nd edn. SpringerVerlag, Berlin. 
Agerer, R. (1999b) Rozites caperatus (Pers.: Fr.) Karst. + Pinus sylvestris L. Descriptions of Ectomycorrhizae 4: 109-113.

Agerer, R. (1999c) Elaphomyces aculeatus Tul. +Quercus robur L. Descriptions of Ectomycorrhizae 4: 37-41.

Agerer, R. (1999d) Gyroporus cyanescens (Bull.: Fr.) Quél. + Pinus sylvestris L. Descriptions of Ectomycorrhizae 4: 43-47.

Agerer, R. (2002a) Elaphomyces aculeatus. In Colour Atlas of Ectomycorrhizae (R. Agerer, ed.): pl. 158. Einhorn Verlag, Schwäbisch Gmünd.

Agerer, R. (2002b) Gomphus clavatus. In Colour Atlas of Ectomycorrhizae (R. Agerer, ed.): pl. 155. Einhorn Verlag, Schwäbisch Gmünd.

Agerer, R. (2002c) Gyroporus cyanescens. In Colour Atlas of Ectomycorrhizae (R. Agerer, ed.): pl. 156. Einhorn Verlag, Schwäbisch Gmünd.

Agerer, R. (2002d) Rozites caperatus. In Colour Atlas of Ectomycorrhizae (R. Agerer, ed.): pl. 157. Einhorn Verlag, Schwäbisch Gmünd.

Agerer, R. (2002e) The ectomycorrhiza Piceirhiza internicrassihyphis: a weak competitor of Cortinarius obtusus? Mycological Progress 1: 291-299.

Agerer, R. \& Ammirati, J. (1998) Polyporoletus sublividus. In Colour Atlas of Ectomycorrhizae (R. Agerer, ed.): pl. 130. Einhorn Verlag, Schwäbisch Gmünd.

Agerer, R. \& Beenken, L. (1998a) Geastrum fimbriatum Fr. + Fagus sylvatica L. Descriptions of Ectomycorrhizae 3: 13-18.

Agerer, R. \& Beenken, L. (1998b) Lyophyllum decastes (Fr.) Sing. + Quercus robur L. Descriptions of Ectomycorrhizae 3: 43-47.

Agerer, R. \& Bougher, N. L. (2001) Tomentella brunneorufa M. J. Larsen + Eucalyptus spec. Descriptions of Ectomycorrhizae 5: 205-212.

Agerer, R. \& Gronbach, E. (1987) Dermocybe cinnamomea. In Colour Atlas of Ectomycorrhizae (R. Agerer, ed.): pl. 7. Einhorn Verlag, Schwäbisch Gmünd.

Agerer, R. \& Gronbach, E. (1988) Cenococcum geophilum. In Colour Atlas of Ectomycorrhizae (R. Agerer, ed.): pl. 11. Einhorn Verlag, Schwäbisch Gmünd.

Agerer, R. \& Gronbach, E. (1989) Paxillus involutus. In Colour Atlas of Ectomycorrhizae (R. Agerer, ed.): pl. 27. Einhorn Verlag, Schwäbisch Gmünd.

Agerer, R. \& Gronbach, E. (1990a) Boletus edulis. In Colour Atlas of Ectomycorrhizae (R. Agerer, ed.): pl. 36. Einhorn Verlag, Schwäbisch Gmünd.

Agerer, R. \& Gronbach, E. (1990b) Xerocomus badius. In Colour Atlas of Ectomycorrhizae (R. Agerer, ed.): pl. 49. Einhorn Verlag, Schwäbisch Gmünd.

Agerer, R. \& Molina, R. (1997) 'Tsugaerhiza luteoannulata' + Tsuga heterophylla (Rafin.) Sarg. Descriptions of Ectomycorrhizae 2: 79-84.

Agerer, R. \& Otto, P. (1997) Bankera fuligineo-alba (J. C. Schmidt: Fr.) Pouzar + Pinus sylvestris L. Descriptions of Ectomycorrhizae 2: $1-6$.

Agerer, R. \& Otto, P. (1998) Bankera fuligineo-alba. In Colour Atlas of Ectomycorrhizae (R. Agerer, ed.): pl. 115. Einhorn Verlag, Schwäbisch Gmünd.

Agerer, R. \& Rambold, G. (1998) DEEMY, a DELTA-based system for characterization and Determination of EctoMYcorrhizae. Version 1.1.

Agerer, R. \& Trappe, J. (1996) 'Tsugaerhiza tripigmentata' + Tsuga heterophylla (Rafin.) Sarg. Descriptions of Ectomycorrhizae 2: 85-89.

Agerer, R. \& Treu, R. (1993) Gyrodon lividus. In Colour Atlas of Ectomycorrhizae (R. Agerer, ed.): pl. 76. Einhorn Verlag, Schwäbisch Gmünd.

Agerer, R. \& Uhl, M. (1989) Dermocybe semisanguinea. In Colour Atlas of Ectomycorrhizae (R. Agerer, ed.): pl. 25. Einhorn Verlag, Schwäbisch Gmünd.

Agerer, R. \& Waller, K. (1993) Tricholoma acerbum. In Colour Atlas of Ectomycorrhizae (R. Agerer, ed.): pl. 82. Einhorn Verlag, Schwäbisch Gmünd.
Agerer, R. \& Weiss, M. (1989) Studies on ectomycorrhizae. XX Mycorrhizae formed by Thelephora terrestris on Norway spruce. Mycologia 81: 444-453.

Agerer, R. \& Weiss, M. (1990) Thelephora terrestris. In Colour Atlas of Ectomycorrhizae (R. Agerer, ed.): pl. 48. Einhorn Verlag, Schwäbisch Gmünd.

Agerer, R., Beenken, L. \& Ammirati, J. (1998b) Polyporoletus sublividus Snell. +Abies amabilis Forb. Descriptions of Ectomycorrhizae 3: 85-91.

Agerer, R., Beenken, L. \& Bougher, N. L. (2001) Descomyces albus (Klotzsch) Bougher \& Castellano + Eucalyptus spec. Descriptions of Ectomycorrhizae 5: 41-47.

Agerer, R., Beenken, L. \& Christan, J. (1998a) Gomphus clavatus (Pers.: Fr.) S. F. Gray + Picea abies (L) Karst. Descriptions of Ectomycorrhizae 3: 25-29.

Agerer, R., Klostermeyer, D., Steglich, W., Franz, F. \& Acker, G. (1996a) Ectomycorrhizae of Albatrellus ovinus (Scutigeraceae) on Norway spruce with some remarks on the systematic position of the family. Mycotaxon 56: 289-307.

Agerer, R., Kraigher, H. \& Javornik, B. (1996b) Identification of ectomycorrhizae of Hydnum rufescens on Norway spruce and the variability of the ITS region of Hydnum rufescens and $H$. repandum (Basidiomycetes). Nova Hedwigia 63: 183-194.

Agerer, R., Müller, W. R. \& Bahnweg, G. (1996c) Ectomycorrhizae of Rhizopogon subcaerulescens on Tsuga heterophylla. Nova Hedwigia 63: 397-415.

Águeda, B., Fernández, M. \& De Miguel, A. M. (2001) Ectomicorrizas presentes en la plantación trufera 'Los Quejigares' (Soria). Libro de Actas del III Congreso Forestal Español, Granada 2001 1: 100-106.

Al Sayegh-Petkovsek, S. \& Kraigher, H. (1999) Black types of ectomycorrhizae on six-month old Norway spruce seedlings. Phyton 39 (3) : 213-217.

Al Sayegh-Petkovsek, S. \& Kraigher, H. (2000) Types of ectomycorrhizae from Kocevska Reka. Phyton 40 (6) : 37-42.

Amicucci, A., Zambonelli, A., Guidi, C. \& Stocchi, V. (2001) Morphological and molecular characterisation of Pulvinulla constellatio ectomycorrhizae. FEMS Microbioloby Letters 194: $121-125$.

Angelini, P. \& Granetti, B. (1995) La micorrizazione di alcuni cloni micropropagati di Populus alba L. con Tuber magnatum Pico. Giornale Botanico Italiano 129: 1161-1177.

Azul, A. M. \& Freitas, H. (1999) Mycorrhizal fungi and their application to forestation programmes with cork oak (Quercus suber L.). In Micorrización en Áreas Mediterráneas de la Peninsula Ibérica. 75-82. Junta de Extremadura, Badajoz.

Azul, A. M., Agerer, R. \& Freitas, H. (1999) 'Quercirhiza nodulosomorpha' + Quercus suber L. Descriptions of Ectomycorrhizae 4: 103-108.

Azul, A. M., Agerer, R. \& Freitas, H. (2001a) 'Quercirhiza ectendotrophica' + Quercus suber L. Descriptions of Ectomycorrhizae 5: 67-72.

Azul, A. M., Agerer, R. \& Freitas, H. (2001b) 'Quercirhiza internangularis' + Quercus suber $\mathrm{L}$. Descriptions of Ectomycorrhizae 5: 79-83.

Azul, A. M., Agerer, R. \& Freitas, H. (2001c) 'Quercirhiza pedicae' + Quercus suber L. Descriptions of Ectomycorrhizae 5: 85-91.

Azul, A. M., Agerer, R. \& Freitas, H. (2001d) 'Quercirhiza russulocystidiata' + Quercus suber L. Descriptions of Ectomycorrhizae 5: 93-98.

Azul, A. M., Agerer, R. \& Freitas, H. (2001e) 'Quercirhiza sclerotiigera' + Quercus suber L. Descriptions of Ectomycorrhizae 5: 99-105.

Baxter, J. W., Pickett, S. T. A., Carreiro, M. M. \& Dighton, J. (1999) Ectomycorrhizal diversity and community structure in oak forest stands exposed to contrasting anthropogenic impacts. Canadian Journal of Botany 77: 771-782.

Becerra, A., Daniele, G., Domínguez, L., Nouhra, E. \& Horton, T. (2002) Ectomycorrhizae between Alnus acuminata H.B.K. and 
Naucoria escharoides (Fr.: Fr.) Kummer from Argentina. Mycorrhiza 12: 61-66.

Becerra, A., Nouhra, E., Daniele, G., Domínguez, L. \& McKay, D. (2005) Ectomycorrhizas of Cortinarius helodes and Gyrodon monticola with Alnus acuminata from Argentina. Mycorrhiza 15: $7-15$.

Beenken, L. (1996a) Inocybe appendiculata. In Colour Atlas of Ectomycorrhizae (R. Agerer, ed.): pl. 94. Einhorn Verlag, Schwäbisch Gmünd.

Beenken, L. (1996b) Inocybe fuscomarginata. In Colour Atlas of Ectomycorrhizae (R. Agerer, ed.): pl. 95. Einhorn Verlag, Schwäbisch Gmünd.

Beenken, L. (1996c) Inocybe obscurobadia. In Colour Atlas of Ectomycorrhizae (R. Agerer, ed.): pl. 96. Einhorn Verlag, Schwäbisch Gmünd.

Beenken, L. (1996d) Inocybe terrigena. In Colour Atlas of Ectomycorrhizae (R. Agerer, ed.): pl. 97. Einhorn Verlag, Schwäbisch Gmünd.

Beenken, L. (2001a) Russula aeruginea Lindbl. ex Fr. + Betula pendula Roth. Descriptions of Ectomycorrhizae 5: 107-113.

Beenken, L. (2001b) Russula alnetorum Romagn. + Alnus viridis (Chaix) DC. Descriptions of Ectomycorrhizae 5: 115-123.

Beenken, L. (2001c) Russula atroglauca Einhellinger + Betula pubescens Ehrh. Descriptions of Ectomycorrhizae 5: 125-130.

Beenken, L. (2001d) Russula cyanoxantha (Schaeff.) Fr. + Fagus sylvatica L. Descriptions of Ectomycorrhizae 5: 131-137.

Beenken, L. (2001e) Russula delica Fr. + Tilia sp. Descriptions of Ectomycorrhizae 5: 139-145.

Beenken, L. (2001f) Russula densifolia Secr. ex Gill. + Fagus sylvatica L. Descriptions of Ectomycorrhizae 5: 147-155.

Beenken, L. (2001g) Russula densifolia Secr. ex Gill. + Picea abies (L.) H. Karst. Descriptions of Ectomycorrhizae 5: 157-161.

Beenken, L. (2001h) Russula foetens (Pers.: Fr.) Fr. + Fagus sylvatica L. Descriptions of Ectomycorrhizae 5: 163-168.

Beenken, L. (2001i) Russula medullata Romagn. + Populus tremula L. Descriptions of Ectomycorrhizae 5: 169-174.

Beenken, L. (2001j) Russula nothofaginea Sing. + Nothofagus dombeyi (Mirbel) Oersted. Descriptions of Ectomycorrhizae 5: 175-179.

Beenken, L. (2001k) Russula versicolor J. Schaeff. + Betula pubescens Ehrh. Descriptions of Ectomycorrhizae 5: 181-185.

Beenken, L. (20011) Russula vesca Fr. + Quercus robur L. Descriptions of Ectomycorrhizae 5: 187-192.

Beenken, L. (2001m) Russula vinosa Lindbl. + Picea abies (L.) H. Karst. Descriptions of Ectomycorrhizae 5: 193-198.

Beenken, L. (2001n) Russula virescens (Schaeff.) Fr. + Quercus robur L. Descriptions of Ectomycorrhizae 5: 199-203.

Beenken, L. \& Agerer, R. (1996) 'Piceirhiza stagonopleres' + Picea abies (L.) Karst. Descriptions of Ectomycorrhizae 1: 71-76.

Beenken, L., Agerer, R. \& Bahnweg, G. (1996a) Inocybe appendiculata Kühn. + Picea abies (L.) Karst. Descriptions of Ectomycorrhizae 1: 35-40.

Beenken, L., Agerer, R. \& Bahnweg, G. (1996b) Inocybe fuscomarginata Kühn. + Salix spec./Populus nigra L. Descriptions of Ectomycorrhizae 1: 41-46.

Beenken, L., Agerer, R. \& Bahnweg, G. (1996c) Inocybe obscurobadia (J. Favre) Grund \& D. E. Stuntz+Picea abies (L.) Karst. Descriptions of Ectomycorrhizae 1: 47-52.

Beenken, L., Agerer, R. \& Bahnweg, G. (1996d) Inocybe terrigena (Fr.) Kuyper + Pinus sylvestris L. Descriptions of Ectomycorrhizae 1: 53-58.

Bencivenga, M., Di Massimo, G., Donnini, D. \& Tanfulli, M. (1995) Micorrize inquinanti frequenti nelle piante tartufigene. Nota 1- Inquinanti in vivaio. Micologia Italiana 1995 (2): 167-178.

Bencivenga, M., Donnini, D. \& Di Massimo, G. (1992) Analisi delle micorrize in una tartufaia coltivata di Tuber melanosporum undici anni dopo l'impianto. Micologia e Vegetazione Mediterranea 7: 159-171.

Bencivenga, M., Tanfulli, M. \& Donnini, D. (1997) Micorrizazione di Quercus cerris L. con Tuber oligospermum (Tul. \& Tul.) Trappe. Micologia Italiana 1997 (3): 89-93.
Berg, B. \& Gronbach, E. (1988) Piceirhiza nigra. In Colour Atlas of Ectomycorrhizae (R. Agerer, ed.): pl. 19. Einhorn Verlag, Schwäbisch Gmünd.

Berg, B. \& Gronbach, E. (1989) Piceirhiza gelatinosa. In Colour Atlas of Ectomycorrhizae (R. Agerer, ed.): pl. 30. Einhorn Verlag, Schwäbisch Gmünd.

Blaschke, H. (1988) Tuber puberulum. In Colour Atlas of Ectomycorrhizae (R. Agerer, ed.): pl. 22. Einhorn Verlag, Schwäbisch Gmünd.

Bradbury, S. M. (1998) Ectomycorrhizas of lodgepole pine (Pinus contorta) seedlings originating from seed in southwestern Alberta cut blocks. Canadian Journal of Botany 76: 213-217.

Bradbury, S. M., Danielson, R. M. \& Visser, S. (1998) Ectomycorrhizas from regenerating stands of lodgepole pine (Pinus contorta). Canadian Journal of Botany 76: 218-227.

Brand, F. (1987a) Lactarius subdulcis. In Colour Atlas of Ectomycorrhizae (R. Agerer, ed.): pl. 5. Einhorn Verlag, Schwäbisch Gmünd.

Brand, F. (1987b) Lactarius vellereus. In Colour Atlas of Ectomycorrhizae (R. Agerer, ed.): pl. 6. Einhorn Verlag, Schwäbisch Gmünd.

Brand, F. (1988a) Fagirhiza cystidiophora. In Colour Atlas of Ectomycorrhizae (R. Agerer, ed.): pl. 15. Einhorn Verlag, Schwäbisch Gmünd.

Brand, F. (1988b) Fagirhiza granulosa. In Colour Atlas of Ectomycorrhizae (R. Agerer, ed.): pl. 16. Einhorn Verlag, Schwäbisch Gmünd.

Brand, F. (1988c) Fagirhiza tubulosa. In Colour Atlas of Ectomycorrhizae (R. Agerer, ed.): pl. 17. Einhorn Verlag, Schwäbisch Gmünd.

Brand, F. (1988d) Laccaria amethystina. In Colour Atlas of Ectomycorrhizae (R. Agerer, ed.): pl. 18. Einhorn Verlag, Schwäbisch Gmünd.

Brand, F. (1989a) Studies on ectomycorrhizae XXI. Beech ectomycorrhizae and rhizomorphs of Xerocomus chrysenteron (Boletales). Nova Hedwigia 48: 469-483.

Brand, F. (1989b) Xerocomus chrysenteron. In Colour Atlas of Ectomycorrhizae (R. Agerer, ed.): pl. 34. Einhorn Verlag, Schwäbisch Gmünd.

Brand, F. (1991a) Fagirhiza spinulosa. In Colour Atlas of Ectomycorrhizae (R. Agerer, ed.): pl. 54. Einhorn Verlag, Schwäbisch Gmünd.

Brand, F. (1991b) Fagirhiza setifera. In Colour Atlas of Ectomycorrhizae (R. Agerer, ed.): pl. 55. Einhorn Verlag, Schwäbisch Gmünd.

Brand, F. (1991c) Russula mairei. In Colour Atlas of Ectomycorrhizae (R. Agerer, ed.): pl. 65. Einhorn Verlag, Schwäbisch Gmünd.

Brand, F. (1991d) Fagirhiza fusca. In Colour Atlas of Ectomycorrhizae (R. Agerer, ed.): pl. 56. Einhorn Verlag, Schwäbisch Gmünd.

Brand, F. (1991e) Genea hispidula. In Colour Atlas of Ectomycorrhizae (R. Agerer, ed.): pl. 57. Einhorn Verlag, Schwäbisch Gmünd.

Brand, F. (1991f) Byssocorticium atrovirens. In Colour Atlas of Ectomycorrhizae (R. Agerer, ed.): pl. 51. Einhorn Verlag, Schwäbisch Gmünd.

Brand, F. (1991g) Elaphomyces muricatus. In Colour Atlas of Ectomycorrhizae (R. Agerer, ed.): pl. 53. Einhorn Verlag, Schwäbisch Gmünd.

Brand, F. (1991h) Lactarius pallidus. In Colour Atlas of Ectomycorrhizae (R. Agerer, ed.): pl. 59. Einhorn Verlag, Schwäbisch Gmünd.

Brand, F. (1991i) Lactarius rubrocinctus. In Colour Atlas of Ectomycorrhizae (R. Agerer, ed.): pl. 60. Einhorn Verlag, Schwäbisch Gmünd.

Brand, F. (1991j) Piloderma croceum. In Colour Atlas of Ectomycorrhizae (R. Agerer, ed.): pl. 62. Einhorn Verlag, Schwäbisch Gmünd.

Brand, F. (1991k) Russula illota. In Colour Atlas of Ectomycorrhizae (R. Agerer, ed.): pl. 64. Einhorn Verlag, Schwäbisch Gmünd. 
Brand, F. (19911) Ektomykorrhizen an Fagus: Charakterisierung und Identifizierung, ökologische Kennzeichnung und unsterile Kultivierung. [Libri Botanici Vol. 2.] IHW-Verlag, Eching.

Brand, F. (1992a) Fagirhiza globulifera. In Colour Atlas of Ectomycorrhizae (R. Agerer, ed.): pl. 70. Einhorn Verlag, Schwäbisch Gmünd.

Brand, F. (1992b) Lactarius acris. In Colour Atlas of Ectomycorrhizae (R. Agerer, ed.): pl. 72. Einhorn Verlag, Schwäbisch Gmünd.

Brand, F. (1992c) Cortinarius bolaris. In Colour Atlas of Ectomycorrhizae (R. Agerer, ed.): pl. 67. Einhorn Verlag, Schwäbisch Gmünd.

Brand, F. (1992d) Cortinarius cinnabarinus. In Colour Atlas of Ectomycorrhizae (R. Agerer, ed.): pl. 68. Einhorn Verlag, Schwäbisch Gmünd.

Brand, F. (1992e) Fagirhiza arachnoidea. In Colour Atlas of Ectomycorrhizae (R. Agerer, ed.): pl. 69. Einhorn Verlag, Schwäbisch Gmünd.

Brand, F. (1992f) Tricholoma sciodes. In Colour Atlas of Ectomycorrhizae (R. Agerer, ed.): pl. 74. Einhorn Verlag, Schwäbisch Gmünd.

Brand, F., Gronbach, E. \& Taylor, A. F.S. (1992) Piceirhiza bicolorata. In Colour Atlas of Ectomycorrhizae (R. Agerer, ed.): pl. 73. Einhorn Verlag, Schwäbisch Gmünd.

Brunner, I., Amiet, R., Zollinger, M. \& Egli, S. (1992) Ectomycorrhizal syntheses with Picea abies and three fungal species: a case study on the use of an in vitro technique to identify naturally occurring ectomycorrhizae. Mycorrhiza 2: 89-96.

Buée, M., Vairelles, D. \& Garbaye, J. (2004) Year-round monitoring of diversity and potential metabolic activity of the ectomycorrhizal community in a beech (Fagus sylvatica) forest subjected to two thinning regimes. Mycorrhiza [online], DOI 10.1007/s00572-0040313-6.

Ceruti, A., Benvenuti, R. \& Luppi Mosca, A. M. (1988) Micorrize di Fagus sylvatica con specie di Lactarius, Russula, Laccaria e Cortinarius. Allionia 28: 125-134.

Ceruti, A., Tozzi, M. \& Reitano, G. (1985) Sintesi micorrizica tra Boletus aereus e Castanea sativa. Allionia 27: 5-9.

Ceruti, A., Tozzi, M. \& Reitano, G. (1987) Micorrize di sintesi tra Boletus edulis, Pinus sylvestris e Picea excelsa. Allionia 28: 117-124.

Chevalier, G. (1973) Synthèse axénique des mycorhizes de Tuber brumale Vitt. a partir de cultures pures du champignon. Annales de phytopathologie 5: 163-182.

Chu-Chou, M. \& Grace, L. J. (1983) Characterization and identification of mycorrhizas of Douglas fir in New Zealand. European Journal of Forest Pathology 13: 251-260.

Comandini, O. \& Pacioni, G. (1997) Mycorrhizae of Asian black truffles, Tuber himalayense and T. indicum. Mycotaxon 63: 77-86.

Comandini, O., Haug, I., Rinaldi, A. C. \& Kuyper, T. W. (2004) Uniting Tricholoma sulphureum and T. bufonium. Mycological Research 108: 1162-1171.

Countess, R. E. \& Goodman, D. M. (2000) Cantharellus formosus Corner + Tsuga heterophylla (Raf.) Sarg. In A Manual of concise Descriptions of North American Ectomycorrhizae: including microscopic and molecular characterization (D. M. Goodman, D. M. Durall, J. A. Trofymow \& S. Berch, eds): CDE 21. Mycologue Publications, Sidney, BC.

Cripps, C. (1997a) Inocybe lacera (Fr.: Fr.) Kumm. + Populus tremuloides Michx. Descriptions of Ectomycorrhizae 2: 19-23.

Cripps, C. (1997b) Tricholoma scalpturatum (Fr.) Quél. + Populus tremuloides Michx. Descriptions of Ectomycorrhizae 2: 73-78.

Cuvelier, J.-J. \& Agerer, R. (1991) Cortinarius armillatus. In Colour Atlas of Ectomycorrhizae (R. Agerer, ed.): pl. 52. Einhorn Verlag, Schwäbisch Gmünd

Dahlberg, A., Jonsson, L., Nylund, J. E. (1997) Species diversity and distribution of biomass above and below ground among ectomycorrhizal fungi in an old-growth Norway spruce forest in South Sweden. Canadian Journal of Botany 75: 1323-1335.

Danielson, R. M. (1984a) Ectomycorrhiza formation by the operculate discomycete Sphaerosporella brunnea (Pezizales). Mycologia 76: $454-461$
Danielson, R. M. (1984b) Ectomycorrhizal associations in jack pine stands in northeastern Alberta. Canadian Journal of Botany 62: 932-939.

Danielson, R. M. \& Pruden, M. (1989) The ectomycorrhizal status of urban spruce. Mycologia 81: 335-341.

Danielson, R. M., Zak, J. C. \& Parkinson, D. (1984) Mycorrhizal inoculum in a peat deposit formed under a white spruce stand in Alberta. Canadian Journal of Botany 63: 2557-2560.

De Román, M. \& De Miguel, A. M. (2005) Post-fire, seasonal and annual dynamics of the ectomycorrhizal community in a Quercus ilex L. forest over a 3-year period. Mycorrhiza online, DOI 10.1007/s00572-005-0353-6.

De Román, M., Agerer, R. \& De Miguel, A. M. (2002a) 'Quercirhiza cumulosa'+Quercus ilex L. subsp. ballota (Desf.) Samp. Descriptions of Ectomycorrhizae 6: 13-18.

De Román, M., Agerer, R. \& De Miguel, A. M. (2002b) 'Quercirhiza stellata' + Quercus ilex L. subsp. ballota (Desf.) Samp. Descriptions of Ectomycorrhizae 6: 19-24.

Di Battista, C., Bouchard, D., Martin, F., Genere, B., Amirault, J.-M. \& Le Tacon, F. (2002) Survival after outplanting of the ectomycorrhizal fungus Laccaria bicolor S238N inoculated on Douglas fir (Pseudotsuga menziesii (Mirb.) Franco) cuttings. Annals of Forest Science 59: 81-92.

Di Massimo, G., García-Montero, L. G., Manjón, J. L. \& Díez, J. (1996) Hongos micorrícicos competidores de Tuber nigrum Bull. (=Tuber melanosporum Vitt.) presentes en ecosistemas naturales y viveros del centro de España. Boletin de la Sociodad Micologica de Madrid 21: 189-199.

Dickie, I. A., Guza, R. C., Krazewski, S. E. \& Reich, P. B. (2004) Shared ectomycorrhizal fungi between a herbaceous perennial (Helianthemum bicknellii) and oak (Quercus) seedlings. New Phytologist 164: 375-382.

Dominik, T. (1969) Key to ectotrophic mycorrhizae. Folia Forestalia Polonica 15: 309-328.

Donnini, D. \& Bencivenga, M. (1995) Micorrize inquinanti frequenti nelle piante tartufigene. Nota 2 - Inquinanti in campo. Micologia Italiana 1995 (2): 185-207.

Duñabeitia, M. K., Hormilla, S., Salcedo, I. \& Peña, J. I. (1996) Ectomycorrhizae synthesized between Pinus radiata and eight fungi associated with Pinus spp. Mycologia 88: 897-908.

Eberhardt, J. L., Oberwinkler, F., Verbeken, A., Rinaldi, A. C., Pacioni, G. \& Comandini, O. (2000) Lactarius ectomycorrhizae on Abies alba: morphological description, molecular characterization and taxonomic remarks. Mycologia 92: 860-873.

Eberhardt, U., Walter, L. \& Kottke, I. (1999) Molecular and morphological discrimination between Tylospora fibrillosa and Tylospora asterophora mycorrhizae. Canadian Journal of Botany 77: 11-21.

Eberhart, J. \& Luoma, D. M. (1996) Truncocolumella citrina Zeller + Pseudotsuga menziesii (Mirb.) Franco. In A Manual of Concise Descriptions of North American Ectomycorrhizae: including microscopic and molecular characterization (D. M. Goodman, D. M. Durall, J. A. Trofymow \& S. Berch, eds): CDE 9. Mycologue Publications, Sidney, BC.

Eberhart, J. \& Luoma, D. M. (1997) Lactarius rubrilacteus Hesler $\&$ Smith + Pseudotsuga menziesii (Mirb.) Franco. In A Manual of Concise Descriptions of North American Ectomycorrhizae: including microscopic and molecular characterization (D. M. Goodman, D. M. Durall, J. A. Trofymow \& S. Berch, eds): CDE 15. Mycologue Publications, Sidney, BC.

Eberhart, J. \& Luoma, D. M. (2000) Russula densifolia (Secr.) Gillet + Pseudotsuga menziesii (Mirb.) Franco. In A Manual of Concise Descriptions of North American Ectomycorrhizae: including microscopic and molecular characterization (D. M. Goodman, D. M. Durall, J. A. Trofymow \& S. Berch, eds): CDE 22. Mycologue Publications, Sidney, BC.

Egli, S., Ayer, F., Peter, M. \& Schneider, B. (2001) Arcangeliella borziana Cavara+Picea abies (L.) Karst. Descriptions of Ectomycorrhizae 5: 9-14. 
Etayo, M. L. \& De Miguel, A. M. (1998) Estudio de las ectomicorrizas en una trufera cultivada situada en Olóriz (Navarra). Publicaciones de Biología de la Universidad de Navarra, serie Botánica 11: 55-114.

Fassi, B. \& De Vecchi, E. (1962) Ricerche sulle micorrize ectotrofiche del pino strobo in vivaio. I. Descrizione di alcune forme piú diffuse in Piemonte. Allionia 8: 133-152.

Fassi, B. \& Fontana, A. (1966) Ricerche sulle micorrize ectotrofiche del pino strobo in vivaio. II. Micorrize di Thelephora terrestris Ehrh. ex Fries, di Laccaria laccata (Scop.) Berk. et Br. e di Hebeloma mesophaeum Pers. ex Fries. Allionia 12: 47-53.

Fischer, C. R. \& Agerer, R. (1996) 'Quercirhiza argenteobrunneola' + Quercus ilex L. Descriptions of Ectomycorrhizae 1: 101-105.

Fischer, Ch., Suz, L., Martin, M. \& Colinas, C. (2004) Tuber brumale Vitt. +Quercus ilex L. Descriptions of Ectomycorrhizae 7-8: 135-141.

Fontana, A. (1961) Primo contributo allo studio delle micorrize dei pioppi in Piemonte. Allionia 7: 87-129.

Fontana, A. (1964) Simbiosi micorrizica di Hebeloma hiemale Bres. con un salice e con un pioppo. Allionia 9: 113-118.

Fontana, A. \& Bonfante-Fasolo, P. (1971) Sintesi micorrizica di Tuber brumale Vitt. con Pinus nigra Arnold. Allionia 17: 15-18.

Fontana, A. \& Centrella, E. (1967) Ectomicorrize prodotte da funghi ipogei. Allionia 13: 149-175.

Fransson, P. (2004a) Craterellus tubaeformis (Fr.) Quél. (syn. Cantharellus tubaeformis Fr.: Fr.) + Quercus robur L. Descriptions of Ectomycorrhizae 7-8: 37-43.

Fransson, P. (2004b) Russula decolorans Fr. + Pinus sylvestris L. Karst. Descriptions of Ectomycorrhizae 7-8: 109-115.

Gardes, M. \& Bruns, T. D. (1996) Community structure of ectomycorrhizal fungi in a Pinus muricata forest: above- and belowground views. Canadian Journal of Botany 74: 1572-1583.

Gill, W. M., Guerin-Laguette, A., Lapeyrie, F. \& Suzuki, K. (2000) Matsutake - morphological evidence of ectomycorrhiza formation between Tricholoma matsutake and host roots in a pure Pinus densiflora forest stand. New Phytologist 147: 381-388.

Gillespie, S. M. K., Durall, D. M. \& Hagerman, S. M. (2000) Thelephora terrestris-like + Betula papyrifera Marsch. In A Manual of Concise Descriptions of North American Ectomycorrhizae: including microscopic and molecular characterization (D. M. Goodman, D. M. Durall, J. A. Trofymow \& S. Berch, eds): CDE 20. Mycologue Publications, Sidney, BC.

Giltrap, N. J. (1982) Hebeloma spp. as mycorrhizal associates of birch. Transactions of the British Mycological Society 79: $157-160$

Giomaro, G., Zambonelli, A., Sisti, D., Cecchini, M., Evangelista, V. \& Stocchi, V. (2000) Anatomical and morphological characterization of mycorrhizas of five strains of Tuber borchii Vittad. Mycorrhiza 10: 107-114.

Giovannetti, G. \& Fontana, A. (1981) Simbiosi micorrizica di Tuber macrosporum Vitt. con alcune Fagales. Allionia 24: 13-17.

Giraud, M. (1988) Prélèvement et analyse de mycorhizes. In La truffe, FNPT 10, Congrès de la Trufficulture, Saintes, 27-28 Novembre 1987: 49-63.

Godbout, C. \& Fortin, J. A. (1983) Morphological features of synthesized ectomycorrhizae of Alnus crispa and A. rugosa. New Phytologist 94: 249-262.

Godbout, C. \& Fortin, J. A. (1985) Synthesized ectomycorrhizae of aspen: fungal genus level of structural characterization. Canadian Journal of Botany 63: 252-262.

Golldack, J., Münzenberger, B. \& Hüttl, R. (1997a) 'Pinirhiza lactariosimilis' + Pinus sylvestris L. Descriptions of Ectomycorrhizae 2: 43-48.

Golldack, J., Münzenberger, B. \& Hüttl, R. (1997b) 'Pinirhiza lactogelatinosa' + Pinus sylvestris L. Descriptions of Ectomycorrhizae 2: 49-53.

Golldack, J., Münzenberger, B. \& Hüttl, R. (1998a) 'Pinirhiza cyaneoviridis' + Pinus sylvestris L. Descriptions of Ectomycorrhizae 3: $49-54$.
Golldack, J., Münzenberger, B. \& Hüttl, R. (1998b) 'Pinirhiza discolor' + Pinus sylvestris L. Descriptions of Ectomycorrhizae 3: 55-60.

Golldack, J., Münzenberger, B. \& Hüttl, R. (1999a) 'Pinirhiza dimorpha' + Pinus sylvestris L. Descriptions of Ectomycorrhizae 4: 73-78.

Golldack, J., Münzenberger, B. \& Hüttl, R. (1999b) 'Pinirhiza flexipila' + Pinus sylvestris L. Descriptions of Ectomycorrhizae 4: 79-84.

Golldack, J., Münzenberger, B. \& Hüttl, R. F. (1996c) Pinirhiza granulosa. In Colour Atlas of Ectomycorrhizae (R. Agerer, ed.): pl. 99. Einhorn Verlag, Schwäbisch Gmünd.

Golldack, J., Münzenberger, B. \& Hüttl, R. F. (1998c) Pinirhiza cyaneoviridis. In Colour Atlas of Ectomycorrhizae (R. Agerer, ed.): pl. 126. Einhorn Verlag, Schwäbisch Gmünd.

Golldack, J., Münzenberger, B. \& Hüttl, R. F. (1998d) Pinirhiza discolor. In Colour Atlas of Ectomycorrhizae (R. Agerer, ed.): pl. 127. Einhorn Verlag, Schwäbisch Gmünd.

Golldack, J., Münzenberger, B., Agerer, R. \& Hüttl, R. (1996a) 'Pinirhiza granulosa'+Pinus sylvestris L. Descriptions of Ectomycorrhizae 1: 77-81.

Golldack, J., Münzenberger, B., Agerer, R. \& Hüttl, R. (1996b) 'Pinirhiza stellannulata' + Pinus sylvestris L. Descriptions of Ectomycorrhizae 1: 89-93.

Golldack, J., Münzenberger, B., Agerer, R. \& Hüttl, R. (1996d) Pinirhiza stellannulata. In Colour Atlas of Ectomycorrhizae (R. Agerer, ed.): pl. 101. Einhorn Verlag, Schwäbisch Gmünd.

Goodman, D. M. (1996a) Tomentella-like + Pseudotsuga menziesii (Mirb.) Franco. In A Manual of Concise Descriptions of North American Ectomycorrhizae: including microscopic and molecular characterization (D. M. Goodman, D. M. Durall, J. A. Trofymow \& S. Berch, eds): CDE 2. Mycologue Publications, Sidney, BC.

Goodman, D. M. (1996b) Unidentified + Pseudotsuga menziesii (Mirb.) Franco. In A Manual of Concise Descriptions of North American Ectomycorrhizae: including microscopic and molecular characterization (D. M. Goodman, D. M. Durall, J. A. Trofymow \& S. Berch, eds): CDE 3. Mycologue Publications, Sidney, BC.

Goodman, D. M. (1996c) Unidentified + Pseudotsuga menziesii (Mirb.) Franco. In A Manual of Concise Descriptions of North American Ectomycorrhizae: including microscopic and molecular characterization (D. M. Goodman, D. M. Durall, J. A. Trofymow \& S. Berch, eds): CDE 4. Mycologue Publications, Sidney, BC.

Goodman, D. M. (1996d) Unidentified + Pseudotsuga menziesii (Mirb.) Franco. In A Manual of Concise Descriptions of North American Ectomycorrhizae: including microscopic and molecular characterization (D. M. Goodman, D. M. Durall, J. A. Trofymow \& S. Berch, eds): CDE 5. Mycologue Publications, Sidney, BC.

Goodman, D. M. (1996e) Rhizopogon vinicolor-like + Pseudotsuga menziesii (Mirb.) Franco. In A Manual of Concise Descriptions of North American Ectomycorrhizae: including microscopic and molecular characterization (D. M. Goodman, D. M. Durall, J. A. Trofymow \& S. Berch, eds): CDE 7. Mycologue Publications, Sidney, BC.

Goodman, D. M. (1996f) Unidentified+Pseudotsuga menziesii (Mirb.) Franco. In A Manual of Concise Descriptions of North American Ectomycorrhizae: including microscopic and molecular characterization (D. M. Goodman, D. M. Durall, J. A. Trofymow \& S. Berch, eds): CDE 8. Mycologue Publications, Sidney, BC.

Goodman, D. M. (1997a) Unidentified + Tsuga heterophylla (Raf.) Sarg. In A Manual of Concise Descriptions of North American Ectomycorrhizae: including microscopic and molecular characterization (D. M. Goodman, D. M. Durall, J. A. Trofymow \& S. Berch, eds): CDE 12. Mycologue Publications, Sidney, BC.

Goodman, D. M. (1997b) Russula-like + Tsuga heterophylla (Raf.) Sarg. In A Manual of Concise Descriptions of North American Ectomycorrhizae: including microscopic and molecular characterization (D. M. Goodman, D. M. Durall, J. A. Trofymow \& S. Berch, eds): CDE 13. Mycologue Publications, Sidney, BC.

Goodman, D. M. (1997c) Unidentified + Tsuga heterophylla (Raf.) Sarg. In A Manual of Concise Descriptions of North American 
Ectomycorrhizae: including microscopic and molecular characterization (D. M. Goodman, D. M. Durall, J. A. Trofymow \& S. Berch, eds): CDE 14. Mycologue Publications, Sidney, BC.

Goodman, D. M. \& Trofymow, J. A. (1996) Piloderma fallax (Libert) Stalpers + Pseudotsuga menziesii (Mirb.) Franco. In A Manual of Concise Descriptions of North American Ectomycorrhizae: including microscopic and molecular characterization (D. M. Goodman, D. M. Durall, J. A. Trofymow \& S. Berch, eds): CDE 1. Mycologue Publications, Sidney, BC.

Goodman, D. M., Durall, D. M., Trofymow, J. A. \& Berch, S. M. (eds). (1996-2000) A Manual of concise Descriptions of North American Ectomycorrhizae. Mycologue Publications, Sidney, BC.

Granetti, B. (1995) Caratteristiche morfologiche, biometriche e strutturali delle micorrize di Tuber di interesse economico. Micologia italiana 1995 (2): 101-117.

Granetti, B. \& Angelini, P. (1992) Competizione tra alcuni funghi ectomicorrizici e Tuber melanosporum in una tartufaia coltivata. Micologia e Vegetazione Mediterranea 7: 173-188.

Granetti, B. \& Baciarelli Falini, L. (1997) Competizione tra le micorrize di Tuber melanosporum Vitt. e quelle di altri funghi in una tartufaia coltivata a Quercus ilex L. Micologia Italiana 1997 (3): $45-59$.

Granetti, B., Angelini, P. \& Rubini, A. (1995a) Morfologia e struttura delle micorrize di Tuber magnatum Pico e Tuber borchii Vitt. con Tilia platyphyllos Scop. Micologia Italiana 1995 (2): 27-34.

Granetti, B., Rubini, A. \& Angelini, P. (1995b) Analisi comparativa morfo-biometrica e strutturale delle micorrize di Tuber aestivum Vitt. con alcune piante forestali. Micologia Italiana 1995 (2): 48-63.

Gronbach, E. (1988) Charakterisierung und Identifizierung von Ektomykorrhizen in einem Fichtenbestand mit Untersuchungen zur Merkmalsvariabilität in sauer beregneten Flächen. Bibliotheca Mycologica 125: 1-217.

Gronbach, E. (1989a) Hygrophorus pustulatus. In Colour Atlas of Ectomycorrhizae (R. Agerer, ed.): pl. 26. Einhorn Verlag, Schwäbisch Gmünd

Gronbach, E. (1989b) Piceirhiza chordata. In Colour Atlas of Ectomycorrhizae (R. Agerer, ed.): pl. 28. Einhorn Verlag, Schwäbisch Gmünd.

Gronbach, E. (1989c) Piceirhiza conspicua. In Colour Atlas of Ectomycorrhizae (R. Agerer, ed.): pl. 29. Einhorn Verlag, Schwäbisch Gmünd.

Gronbach, E. (1989d) Piceirhiza glutinosa. In Colour Atlas of Ectomycorrhizae (R. Agerer, ed.): pl. 31. Einhorn Verlag, Schwäbisch Gmünd.

Gronbach, E. (1989e) Piceirhiza guttata. In Colour Atlas of Ectomycorrhizae (R. Agerer, ed.): pl. 32. Einhorn Verlag, Schwäbisch Gmünd.

Gronbach, E. \& Agerer, R. (1988) Cortinarius obtusus. In Colour Atlas of Ectomycorrhizae (R. Agerer, ed.): pl. 12. Einhorn Verlag, Schwäbisch Gmünd.

Gronbach, E. \& Berg, B. (1988) Piceirhiza obscura. In Colour Atlas of Ectomycorrhizae (R. Agerer, ed.): pl. 20. Einhorn Verlag, Schwäbisch Gmünd.

Gutiérrez, A., Morte, A. \& Honrubia, M. (2003) Morphological characterization of the mycorrhiza formed by Helianthemum almeriense Pau with Terfezia claveryi Chatin and Picoa lefebvrei (Pat.) Maire. Mycorrhiza 13: 299-307.

Hagerman, S. M., Jones, M. D., Bradfield, G. E., Gillespie, M. \& Durall, D. M. (1999) Effects of clear-cut logging on the diversity and persistence of ectomycorrhizae at a subalpine forest. Canadian Journal of Forest Research 29: 124-134.

Hagerman, S. M., Sakakibara, S. M. \& Durall, D. M. (2001) The potential of woody understory plants to provide refuge for ectomycorrhizal inoculum at an interior Douglas-fir forest after clear-cut logging. Canadian Journal of Forest Research 31: 711-721.

Hahn, C. (2001) Boletus rhodoxanthus Kallenb. + Cistus cf. ladanifer L. Descriptions of Ectomycorrhizae 5: 15-22.

Harniman, S. M. K. \& Durall, D. M. (1996a) Amphinema byssoideslike + Picea engelmannii (Parry) Engelm. In A Manual of Concise
Descriptions of North American Ectomycorrhizae: including microscopic and molecular characterization (D. M. Goodman, D. M. Durall, J. A. Trofymow \& S. Berch, eds): CDE 6. Mycologue Publications, Sidney, BC.

Harniman, S. M. K. \& Durall, D. M. (1996b) Cenococcum geophilum Fr. + Picea engelmannii (Parry) Engelm. In A Manual of Concise Descriptions of North American Ectomycorrhizae: including microscopic and molecular characterization (D. M. Goodman, D. M. Durall, J. A. Trofymow \& S. Berch, eds): CDE 10. Mycologue Publications, Sidney, BC.

Haug, I. (2002a) Hebeloma velutipes. In Colour Atlas of Ectomycorrhizae (R. Agerer, ed.): pl. 150. Einhorn Verlag, Schwäbisch Gmünd.

Haug, I. (2002b) Identification of Picea-ectomycorrhizae by comparing DNA-sequences. Mycological Progress 1: 167-178.

Haug, I., Weber, R., Oberwinkler, F. \& Tschen, J. (1994) The mycorrhizal status of Taiwanese trees and the description of some ectomycorrhizal types. Trees 8 : 237-253.

Haug, I., Weiß, M., Homeier, J., Oberwinkler, F. \& Kottke, I. (2005) Russulaceae and Thelephoraceae form ectomycorrhizas with members of the Nyctaginaceae (Caryophyllales) in the tropical mountain rain forest of southern Ecuador. New Phytologist 165: 923-936.

Hoch, M., Goodman, D. M. \& Chyplyk, C. (1998) Suillus caerulescens A.H. Smith \& Thiers + Pseudotsuga menziesii (Mirb.) Franco. In A Manual of Concise Descriptions of North American Ectomycorrhizae: including microscopic and molecular characterization (D. M. Goodman, D. M. Durall, J. A. Trofymow \& S. Berch, eds): CDE 19. Mycologue Publications, Sidney, BC.

Ingleby, K. (1999a) Inocybe avellana Horak + Shorea leprosula Miq. Descriptions of Ectomycorrhizae 4: 55-60.

Ingleby, K. (1999b) Scleroderma sinnamarense Mont. + Gnetum africanum Welw. Descriptions of Ectomycorrhizae 4: 127-133.

Ingleby, K., Mason, P. A., Last, F. T. \& Fleming, L. V. (1990) Identification of Ectomycorrhizas. Institute of Terrestrial Ecology Research Publication No. 5. HMSO, London.

Jakucs, E. (1998) 'Fagirhiza vermiculiformis' + Fagus sylvatica L. Descriptions of Ectomycorrhizae 3: 7-11.

Jakucs, E. (2001) 'Quercirhiza alboviolacea' + Quercus robur L. Descriptions of Ectomycorrhizae 5: 61-65.

Jakucs, E. (2002a) Ectomycorrhizae of Populus alba L. in South Hungary. Phyton Botanicae 42 (2): 199-210.

Jakucs, E. (2002b) Hebeloma ammophilum. In Colour Atlas of Ectomycorrhizae (R. Agerer, ed.): pl. 145. Einhorn Verlag, Schwäbisch Gmünd.

Jakucs, E. (2002c) Inocybe heimii. In Colour Atlas of Ectomycorrhizae (R. Agerer, ed.): pl. 146. Einhorn Verlag, Schwäbisch Gmünd.

Jakucs, E. (2002d) Lactarius controversus. In Colour Atlas of Ectomycorrhizae (R. Agerer, ed.): pl. 143. Einhorn Verlag, Schwäbisch Gmünd.

Jakucs, E. (2002e) Quercirhiza alboviolacea. In Colour Atlas of Ectomycorrhizae (R. Agerer, ed.): pl. 148. Einhorn Verlag, Schwäbisch Gmünd.

Jakucs, E. (2002f) Russula amoenolens. In Colour Atlas of Ectomycorrhizae (R. Agerer, ed.): pl. 142. Einhorn Verlag, Schwäbisch Gmünd.

Jakucs, E. (2002g) Scleroderma bovista. In Colour Atlas of Ectomycorrhizae (R. Agerer, ed.): pl. 144. Einhorn Verlag, Schwäbisch Gmünd.

Jakucs, E. (2002h) Tomentella pilosa. In Colour Atlas of Ectomycorrhizae (R. Agerer, ed.): pl. 141. Einhorn Verlag, Schwäbisch Gmünd.

Jakucs, E. (2002i) Tomentella subtestacea. In Colour Atlas of Ectomycorrhizae (R. Agerer, ed.): pl. 147. Einhorn Verlag, Schwäbisch Gmünd.

Jakucs, E. (2002j) Xerocomus lanatus. In Colour Atlas of Ectomycorrhizae (R. Agerer, ed.): pl. 149. Einhorn Verlag, Schwäbisch Gmünd. 
Jakucs, E. \& Agerer, R. (1998) Fagirhiza vermiculiformis. In Colour Atlas of Ectomycorrhizae (R. Agerer, ed.): pl. 118. Einhorn Verlag, Schwäbisch Gmünd.

Jakucs, E. \& Agerer, R. (1999a) Scleroderma bovista Fr.+ Populus alba L. Descriptions of Ectomycorrhizae 4: 121-126.

Jakucs, E. \& Agerer, R. (1999b) Tomentella pilosa (Burt) Bourdot \& Galzin + Populus alba L. Descriptions of Ectomycorrhizae 4: 135-140.

Jakucs, E. \& Agerer, R. (2001) Tomentella subtestacea Bourdot \& Galzin + Populus alba L. Descriptions of Ectomycorrhizae 5: 213-219.

Jakucs, E. \& Beenken, L. (1999) Russula amoenolens Romagn. + Populus alba L. Descriptions of Ectomycorrhizae 4: 115-119.

Jakucs, E. \& Beenken, L. (2001) Xerocomus lanatus (Rostk.) Sing. +Quercus cerris L. Descriptions of Ectomycorrhizae 5: 221-225.

Jakucs, E. \& Bratek, Z. (1998a) Genea verrucosa. In Colour Atlas of Ectomycorrhizae (R. Agerer, ed.): pl. 120. Einhorn Verlag, Schwäbisch Gmünd.

Jakucs, E. \& Bratek, Z. (1998b) Rhizopogon vulgaris var. intermedius. In Colour Atlas of Ectomycorrhizae (R. Agerer, ed.): pl. 136. Einhorn Verlag, Schwäbisch Gmünd.

Jakucs, E., Agerer, R. \& Bratek, Z. (1997) 'Quercirhiza fibulocystidiata' +Quercus spec. Descriptions of Ectomycorrhizae 2: 67-71.

Jakucs, E., Agerer, R. \& Bratek, Z. (1998c) Quercirhiza fibulocystidiata. In Colour Atlas of Ectomycorrhizae (R. Agerer, ed.): pl. 132. Einhorn Verlag, Schwäbisch Gmünd.

Jakucs, E., Bratek, Z. \& Agerer, R. (1998a) Genea verrucosa Vitt. + Quercus spec. Descriptions of Ectomycorrhizae 3: 7-11.

Jakucs, E., Bratek, Z., Beenken, L. \& Agerer, R. (1998b) Rhizopogon vulgaris (Vitt.) M. Lange var. intermedius Svrcek + Pinus nigra Arn. Descriptions of Ectomycorrhizae 3: 111-116.

Jakucs, E., Kovács, G. M., Agerer, R. Romsics, C. \& Eros-Honti, Z. (2004) Morphological-anatomical characterization and molecular identification of Tomentella stuposa ectomycorrhizae and related anatomotypes. Mycorrhiza [online]: DOI 10.1007/s00572-0040326-1.

Jakucs, E., Kovács, G. M., Szedlay, G. \& Eros-Honti, Z. (2005) Morphological and molecular diversity and abundance of tomentelloid ectomycorrhizae in broad-leaved forests of the Hungarian Plain. Mycorrhiza [online]: DOI 10.1007/s00572-0050351-8.

Jakucs, E., Magyar, L. \& Beenken, L. (1999) Hebeloma ammophilum Bohus + Fumana procumbens (Dun.) Gr. Godr. Descriptions of Ectomycorrhizae 4: 49-54.

Jakucs, E., Majoros, E. \& Beenken, L. (2001) Lactarius controversus Pers. + Populus alba L. Descriptions of Ectomycorrhizae 5: 55-59.

Jany, J.-L., Garbaye, J. \& Martin, F. (2002) Cenococcum geophilum populations show a high degree of genetic diversity in beech forests. New Phytologist 154: 651-659.

Jones, M. D., Durall, D. M., Harniman, S. M. K., Classen, D. \& Simard, S. W. (1997) Ectomycorrhizal diversity on Betula papyrifera and Pseudotsuga menziesii seedlings grown in the greenhouse or outplanted in single-species and mixed plots in southern British Columbia. Canadian Journal of Forest Research 27: 1872-1889.

Kaldorf, M., Renker, C., Fladung, M. \& Buscot, F. (2004) Characterization and spatial distribution of ectomycorrhizas colonizing aspen clones released in an experimental field. Mycorrhiza 14: 295-306.

Kernaghan, G. (2001) Ectomycorrhizal fungi at tree line in the Canadian Rockies. II. Identification of ectomycorrhizae by anatomy and PCR. Mycorrhiza 10: 217-229.

Kernaghan, G. \& Berch, S. M. (1997) Lactarius scrobiculatus (Fr.) Fr. + Tsuga heterophylla (Raf.) Sarg. In A Manual of Concise Descriptions of North American Ectomycorrhizae: including microscopic and molecular characterization (D. M. Goodman, D. M. Durall, J. A. Trofymow \& S. Berch, eds): CDE 11. Mycologue Publications, Sidney, BC.
Kernaghan, G., Currah, R. S. \& Bayer, R. J. (1997) Russulaceous ectomycorrhizae of Abies lasiocarpa and Picea engelmannii. Canadian Journal of Botany 75: 1843-1850.

Kovács, G. \& Jakucs, E. (2001) 'Helianthemirhiza hirsuta'+ Helianthemum ovatum (Viv.) Dun. Descriptions of Ectomycorrhizae 5: 49-53.

Kovács, G., Jakucs, E., Díez, J., Manjón, J. L. \& Esteve-Raventós, F. (2002) Cortinarius hinnuleus Fr. + Betula celtiberica Rothm. \& Vasc. Descriptions of Ectomycorrhizae 6: 7-11.

Kraigher, H. \& Agerer, R. (1996) Hydnum rufescens. In Colour Atlas of Ectomycorrhizae (R. Agerer, ed.): pl. 92. Einhorn Verlag, Schwäbisch Gmünd.

Kraigher, H., Agerer, R. \& Javornik, B. (1995) Ectomycorrhizae of Lactarius lignyotus on Norway spruce, characterized by anatomical and molecular tools. Mycorrhiza 5: 175-180.

Kuss, P., Raidl, S. \& Beenken, L. (2004) Cortinarius huronensis Ammirati \& Smith var. huronensis + Pinus rotundata Link. Descriptions of Ectomycorrhizae 7-8: 21-27.

Lefevre, C. K. \& Müller, W. R. (1998) Tricholoma magnivelare (Peck) Redhead + Pinus contorta Dougl. var. latifolia Engelm. In $A$ Manual of Concise Descriptions of North American Ectomycorrhizae: including microscopic and molecular characterization (D. M. Goodman, D. M. Durall, J. A. Trofymow \& S. Berch, eds): CDE 18. Mycologue Publications, Sidney, BC.

Luppi, A. M. \& Gautero, C. (1967) Ricerche sulle micorrize di Quercus robur, Q. petraea e $Q$. pubescens in Piemonte. Allionia 13: 129-148.

Magyar, L., Beenken, L. \& Jakucs, E. (1999) Inocybe heimii Bon + Fumana procumbens (Dun.) Gr. Godr. Descriptions of Ectomycorrhizae 4: 61-65.

Massicotte, H. B., Melville, L. H., Peterson, R. L. \& Molina, R. (1999a) Biology of the ectomycorrhizal fungal genus, Rhizopogon. IV. Comparative morphology and anatomy of ectomycorrhizas synthesized between several Rhizopogon species on Ponderosa pine (Pinus ponderosa). New Phytologist 142: 355-370.

Massicotte, H. B., Melville, L. H., Peterson, R. L. \& Molina, R. (2000) Comparative anatomy of ectomycorrhizas synthesized on Douglas fir by Rhizopogon spp. and the hypogeous relative Truncocolumella citrina. New Phytologist 147: 389-400.

Massicotte, H. B., Molina, R., Tackaberry, L. E., Smith, J. \& Amaranthus, M.P. (1999b) Diversity and host specificity of ectomycorrhizal fungi retrieved from three adjacent forest sites by five host species. Canadian Journal of Botany 77: 1053-1076.

Mello, A., Fontana, A., Meotto, F., Comandini, O. \& Bonfante, P. (2001) Molecular and morphological characterization of Tuber magnatum mycorrhizas in a long-term survey. Microbiological Research 155: 279-284.

Meotto, F. (1988) Laccaria laccata (Scop. ex Fr.) Berk. \& Br. and Acacia dealbata Link ectomycorrhizae. Allionia 28: 103-108.

Meotto, F. \& Carraturo, P. (1988) Ectomicorrizia di Sphaerosporella brunnea (A. \& S.) Svrček \& Kubicka in piantine tartufigene. Allionia 28: 109-116.

Meotto, F. \& Pellegrino, S. (1989) Comportamento in campo di querce e castagni micorrizati con Boletus edulis Bull. L'Informatore Agrario 47: 57-61.

Meotto, F., Mello, A., Nosenzo, C. \& Vezzola, V. (1997) Morphological and molecular characterization of Amanita caesarea ectomycorrhizas. Allionia 35: 87-93.

Meotto, F., Nosenzo, C. \& Fontana, A. (1995) Le micorrize delle specie pregiate di Tuber. L'Informatore Agrario 31: 41-45.

Meotto, F., Pellegrino, S. \& Craddock, J. H. (1994) Funghi ectomicorrizici del castagno con particolare referimento ai funghi eduli. Italus Hortus 1: 58-64.

Miller, S. L., Koo, C. D. \& Molina, R. (1990) Characterization of red alder ectomycorrhizae: a preface to monitoring belowground ecological responses. Canadian Journal of Botany 69: 516-531.

Mleczko, P. (1996a) 'Pinirhiza lutea' + Pinus sylvestris L. Descriptions of Ectomycorrhizae 1: 83-88. 
Mleczko, P. (1996b) Pinirhiza lutea. In Colour Atlas of Ectomycorrhizae (R. Agerer, ed.): pl. 100. Einhorn Verlag, Schwäbisch Gmünd.

Mleczko, P. (1997a) Paxillus involutus (Batsch) Fr. + Pinus sylvestris L. Descriptions of Ectomycorrhizae 2: 25-30.

Mleczko, P. (1997b) 'Populirhiza pustulosa'+Populus tremula L. Descriptions of Ectomycorrhizae 2: 61-66.

Mleczko, P. (1998a) 'Pinirhiza globulifera'+Pinus sylvestris L. Descriptions of Ectomycorrhizae 3: 61-66.

Mleczko, P. (1998b) 'Pinirhiza gomphidioidea'+Pinus sylvestris L. Descriptions of Ectomycorrhizae 3: 67-72.

Mleczko, P. (1998c) Pinirhiza gomphidioidea. In Colour Atlas of Ectomycorrhizae (R. Agerer, ed.): pl. 128. Einhorn Verlag, Schwäbisch Gmünd.

Mleczko, P. (1998d) Populirhiza pustulosa. In Colour Atlas of Ectomycorrhizae (R. Agerer, ed.): pl. 131. Einhorn Verlag, Schwäbisch Gmünd.

Mleczko, P. (2002a) Amanita citrina. In Colour Atlas of Ectomycorrhizae (R. Agerer, ed.): pl. 159. Einhorn Verlag, Schwäbisch Gmünd.

Mleczko, P. (2002b) Cantharellus cibarius. In Colour Atlas of Ectomycorrhizae (R. Agerer, ed.): pl. 160. Einhorn Verlag, Schwäbisch Gmünd.

Mleczko, P. (2002c) Craterellus tubaeformis. In Colour Atlas of Ectomycorrhizae (R. Agerer, ed.): pl. 161. Einhorn Verlag, Schwäbisch Gmünd.

Mleczko, P. (2002d) Pinirhiza amyloidea. In Colour Atlas of Ectomycorrhizae (R. Agerer, ed.): pl. 162. Einhorn Verlag, Schwäbisch Gmünd.

Mleczko, P. (2002e) Pinirhiza crystallonitida. In Colour Atlas of Ectomycorrhizae (R. Agerer, ed.): pl. 163. Einhorn Verlag, Schwäbisch Gmünd.

Mleczko, P. (2002f) Pinirhiza ligulata. In Colour Atlas of Ectomycorrhizae (R. Agerer, ed.): pl. 164. Einhorn Verlag, Schwäbisch Gmünd.

Mleczko, P. (2002g) Pinirhiza luteomicacea. In Colour Atlas of Ectomycorrhizae (R. Agerer, ed.): pl. 165. Einhorn Verlag, Schwäbisch Gmünd.

Mleczko, P. (2002h) Rhodocollybia butyracea. In Colour Atlas of Ectomycorrhizae (R. Agerer, ed.): pl. 166. Einhorn Verlag, Schwäbisch Gmünd.

Mleczko, P. (2002i) Russula nigricans. In Colour Atlas of Ectomycorrhizae (R. Agerer, ed.): pl. 167. Einhorn Verlag, Schwäbisch Gmünd.

Mleczko, P. (2002j) Russula sardonia. In Colour Atlas of Ectomycorrhizae (R. Agerer, ed.): pl. 168. Einhorn Verlag, Schwäbisch Gmünd.

Mleczko, P. (2004a) Amanita citrina (Schaeff.) S. F. Gray + Pinus sylvestris L. Descriptions of Ectomycorrhizae 7-8: 1-10.

Mleczko, P. (2004b) Cantharellus cibarius Fr.+Pinus sylvestris L. Descriptions of Ectomycorrhizae 7-8: 11-20.

Mleczko, P. (2004c) Craterellus tubaeformis (Bull.) Quél. + Pinus sylvestris L. Descriptions of Ectomycorrhizae 7-8: 29-36.

Mleczko, P. (2004d) Pinirhiza amyloidea+Pinus sylvestris L. Descriptions of Ectomycorrhizae 7-8: 59-68.

Mleczko, P. (2004e) Pinirhiza crystallonitida+Pinus sylvestris L. Descriptions of Ectomycorrhizae 7-8: 69-77.

Mleczko, P. (2004f) Pinirhiza ligulata + Pinus sylvestris L. Descriptions of Ectomycorrhizae 7-8: 79-86.

Mleczko, P. (2004g) Pinirhiza luteomicacea+Pinus sylvestris L. Descriptions of Ectomycorrhizae 7-8: 87-94.

Mleczko, P. (2004h) Rhodocollybia butyracea (Bull.: Fr.) Lennox (forma butyracea) + Pinus sylvestris L. Descriptions of Ectomycorrhizae 7-8: 101-108.

Mleczko, P. (2004i) Russula nigricans Fr.+Pinus sylvestris L. Descriptions of Ectomycorrhizae 7-8: 117-125.

Mleczko, P. (2004j) Russula sardonia Fr.+Pinus sylvestris L. Descriptions of Ectomycorrhizae 7-8: 127-134.

Mohan, V., Natarajan, K. \& Ingleby, K. (1993) Anatomical studies on ectomycorrhizas. III. The ectomycorrhizas produced by Rhizopogon luteolus and Scleroderma citrinum on Pinus patula. Mycorrhiza 3: 51-56.

Montecchio, L. \& Agerer, R. (1997) 'Piceirhiza cornuta' + Picea abies (L) Karst. Descriptions of Ectomycorrhizae 2: 31-35.

Montecchio, L., Rossi, S. \& Causin, R. (2001b) 'Quercirhiza incrustata' + Quercus ilex L. Descriptions of Ectomycorrhizae 5: 73-78.

Montecchio, L., Rossi, S., Grendene, A. \& Causin, R. (2001a) Cortinarius ionochlorus $\mathrm{R}$. Maire + Quercus ilex L. Descriptions of Ectomycorrhizae 5: 35-40.

Montecchio, L., Rossi, S., Grendene, A. \& Causin, R. (2002) Amphinema byssoides (Pers.: Fr.) J. Erikss.+Quercus ilex L. Descriptions of Ectomycorrhizae 6: 1-6.

Montecchio, L., Rossi, S., Grendene, A. \& Causin, R. (2004) Quercirhiza cistidiophora + Quercus ilex L. Descriptions of Ectomycorrhizae. 7-8: 95-100.

Montecchio, L., Rossi, S., Grendene, A., Causin, R. \& Mutto Accordi, S. (1999a) 'Quercirhiza araneosa' +Quercus ilex L. Descriptions of Ectomycorrhizae 4: 91-96.

Montecchio, L., Rossi, S., Grendene, A., Causin, R. \& Mutto Accordi, S. (1999b) 'Quercirhiza bicolor'+Quercus ilex L. Descriptions of Ectomycorrhizae 4: 97-102.

Montecchio, L., Rossi, S., Grendene, A., Causin, R. \& Mutto Accordi, S. (1998a) 'Quercirhiza sublutea'+Quercus ilex L. Descriptions of Ectomycorrhizae 3: 93-98.

Montecchio, L., Rossi, S., Grendene, A., Causin, R. \& Mutto Accordi, S. (1998b) Quercirhiza sublutea. In Colour Atlas of Ectomycorrhizae (R. Agerer, ed.): pl. 133. Einhorn Verlag, Schwäbisch Gmünd.

Moser, A. M., Petersen, C. A., D'Allura, J. A. \& Southworth, D. (2005) Comparison of ectomycorrhizas of Quercus garryana (Fagaceae) on serpentine and non-serpentine soils in southwestern Oregon. American Journal of Botany 92: 224-230.

Moyersoen, B. (1996a) 'Tetraberliniaerhiza bicolor' + Tetraberlinia bifoliolata (Harms) Hauman. Descriptions of Ectomycorrhizae 1: 137-141.

Moyersoen, B. (1996b) 'Tetraberliniaerhiza cerviformis' + Tetraberlinia bifoliolata (Harms) Hauman. Descriptions of Ectomycorrhizae 1: 143-147.

Moyersoen, B. (1996c) 'Tetraberliniaerhiza heterocystidiae' + Tetraberlinia bifoliolata (Harms) Hauman. Descriptions of Ectomycorrhizae 1: 149-153.

Moyersoen, B. (1996d) Tetraberliniaerhiza bicolor. In Colour Atlas of Ectomycorrhizae (R. Agerer, ed.): pl. 107. Einhorn Verlag, Schwäbisch Gmünd.

Moyersoen, B. (1996e) Tetraberliniaerhiza cerviformis. In Colour Atlas of Ectomycorrhizae (R. Agerer, ed.): pl. 108. Einhorn Verlag, Schwäbisch Gmünd.

Moyersoen, B. (1996f) Tetraberliniaerhiza heterocystidiae. In Colour Atlas of Ectomycorrhizae (R. Agerer, ed.): pl. 109. Einhorn Verlag, Schwäbisch Gmünd.

Moyersoen, B. \& Beever, R. E. (2004) Abundance and characteristics of Pisolithus ectomycorrhizas in New Zealand geothermal areas. Mycologia 96: 1225-1232.

Müller, W. (1994) Betulaerhiza hystrix. In Colour Atlas of Ectomycorrhizae (R. Agerer, ed.): pl. 83. Einhorn Verlag, Schwäbisch Gmünd.

Müller, W. \& Agerer, R. (1990) Studies on ectomycorrhizae XXXI. Betulaerhiza hystrix, an unidentified mycorrhiza on Betula pendula. Cryptogamic Botany 2: 64-68.

Müller, W. \& Agerer, R. (1991) Leccinum scabrum. In Colour Atlas of Ectomycorrhizae (R. Agerer, ed.): pl. 61. Einhorn Verlag, Schwäbisch Gmünd.

Müller, W. R. \& Agerer, R. (1996a) Hysterangium crassirhachis Zeller \& Dodge + Pseudotsuga menziesii (Mirb.) Franco. Descriptions of Ectomycorrhizae 1: 29-34.

Müller, W. R. \& Agerer, R. (1996b) 'Pseudotsugaerhiza baculifera' + Pseudotsuga menziesii (Mirb.) Franco. Descriptions of Ectomycorrhizae 1: 95-100. 
Müller, W. R. \& Agerer, R. (1996c) Hysterangium crassirhachis. In Colour Atlas of Ectomycorrhizae (R. Agerer, ed.): pl. 93. Einhorn Verlag, Schwäbisch Gmünd.

Müller, W. R. \& Agerer, R. (1996d) Pseudotsugaerhiza baculifera. In Colour Atlas of Ectomycorrhizae (R. Agerer, ed.): pl. 102. Einhorn Verlag, Schwäbisch Gmünd.

Müller, W. R., Rauscher, T., Agerer, R. \& Chevalier, G. (1996a) Tuber aestivum Vitt. + Corylus avellana L. Descriptions of Ectomycorrhizae 1: 167-172.

Müller, W. R., Rauscher, T., Agerer, R. \& Chevalier, G. (1996b) Tuber uncinatum Chat. + Corylus avellana L. Descriptions of Ectomycorrhizae 1: 179-183.

Münzenberger, B., Metzler, B., Kottke, I. \& Oberwinkler, F. (1986) Morphologische und anatomische Charakterisierung der Mykorrhiza Lactarius deterrimus-Picea abies in vitro. Zeitschrift für Mykologie 52: 407-422.

Nouhra, E. R., Horton, T. R., Cazares, E. \& Castellano, M. (2005) Morphological and molecular characterization of selected Ramaria mycorrhizae. Mycorrhiza 15: 55-59.

Nuytinck, J., Verbeken, A., Rinaldi, A. C., Leonardi, M., Pacioni, G. \& Comandini, O. (2004) Characterization of Lactarius tesquorum ectomycorrhizae on Cistus sp. and molecular phylogeny of related European Lactarius taxa. Mycologia 96: 272-282.

Palenzona, M. (1969) Sintesi micorrizica tra Tuber aestivum Vitt., Tuber brumale Vitt., Tuber melanosporum Vitt. e semenzali di Corylus avellana L. Allionia 15: 121-131.

Palenzona, M. \& Fontana, A. (1978) Synthèse des mycorhizes de Tuber magnatum Pico avec semis de Quercus pubescens Willd. Mushroom Science X (Part I), Proceedings of the 10th International Congress on the Science and Cultivation of Edible Fungi: 1007-1012.

Palenzona, M., Chevalier, G. \& Fontana, A. (1972) Sintesi micorrizica tra i miceli in coltura di Tuber brumale, T. melanosporum, T. rufum e semenzali di conifere e latifoglie. Allionia 18: 41-52.

Palfner, G. (1995a) Quercirhiza squamosa. In Colour Atlas of Ectomycorrhizae (R. Agerer, ed.): pl. 86. Einhorn Verlag, Schwäbisch Gmünd.

Palfner, G. (1995b) Xerocomus subtomentosus. In Colour Atlas of Ectomycorrhizae (R. Agerer, ed.): pl. 90. Einhorn Verlag, Schwäbisch Gmünd.

Palfner, G. (1996a) Thaxterogaster albocanus Horak \& Moser + Nothofagus pumilio (Poepp. et Endl.) Krasser. Descriptions of Ectomycorrhizae 1: 155-160.

Palfner, G. (1996b) Thaxterogaster albocanus. In Colour Atlas of Ectomycorrhizae (R. Agerer, ed.): pl. 110. Einhorn Verlag, Schwäbisch Gmünd.

Palfner, G. (1997) Descolea antarctica Singer + Nothofagus alpina (Poepp. et Endl.) Oerst. Descriptions of Ectomycorrhizae 2: 7-12.

Palfner, G. (1998a) Balsamia alba. In Colour Atlas of Ectomycorrhizae (R. Agerer, ed.): pl. 114. Einhorn Verlag, Schwäbisch Gmünd.

Palfner, G. (1998b) Descolea antarctica. In Colour Atlas of Ectomycorrhizae (R. Agerer, ed.): pl. 116. Einhorn Verlag, Schwäbisch Gmünd.

Palfner, G. (1998c) Lactarius chrysorrheus. In Colour Atlas of Ectomycorrhizae (R. Agerer, ed.): pl. 121. Einhorn Verlag, Schwäbisch Gmünd.

Palfner, G. (1998d) Leucangium carthusianum. In Colour Atlas of Ectomycorrhizae (R. Agerer, ed.): pl. 123. Einhorn Verlag, Schwäbisch Gmünd.

Palfner, G. (2001) Taxonomische Studien an Ektomykorrhizen aus den Nothofagus-Wäldern Mittelsüdchiles. Bibliotheca Mycologica 190: $1-243$.

Palfner, G. (2002a) Austropaxillus boletinoides. In Colour Atlas of Ectomycorrhizae (R. Agerer, ed.): pl. 151. Einhorn Verlag, Schwäbisch Gmünd.

Palfner, G. (2002b) Cortinarius austrosalor. In Colour Atlas of Ectomycorrhizae (R. Agerer, ed.): pl. 152. Einhorn Verlag, Schwäbisch Gmünd

Palfner, G. (2002c) Cortinarius magellanicus. In Colour Atlas of Ectomycorrhizae (R. Agerer, ed.): pl. 153. Einhorn Verlag, Schwäbisch Gmünd.
Palfner, G. (2002d) Gautieria inapire. In Colour Atlas of Ectomycorrhizae (R. Agerer, ed.): pl. 154. Einhorn Verlag, Schwäbisch Gmünd.

Palfner, G. \& Agerer, A. (1998a) Balsamia alba Harkness + Pinus jeffreyi Grev. \& Balf. Descriptions of Ectomycorrhizae 3: 1-6.

Palfner, G. \& Agerer, R. (1996) Quercirhiza squamosa eine nichtidentifizierte Ektomykorrhiza an Quercus robur. Sendtnera 3: 137-145.

Palfner, G. \& Agerer, R. (1998b) Leucangium carthusianum (Tul.) Paol. + Pseudotsuga menziesii (Mirb.) Franco. Descriptions of Ectomycorrhizae 3: 37-42.

Palfner, G. \& Godoy, R. (1996a) 'Notofagirhiza vinicolor' + Nothofagus pumilio (Poepp. et Endl.) Krasser. Descriptions of Ectomycorrhizae 1: 65-70.

Palfner, G. \& Godoy, R. (1996b) Russula fuegiana Singer + Nothofagus pumilio (Poepp. et Endl.) Krasser. Descriptions of Ectomycorrhizae 1: 131-136.

Palfner, G. \& Godoy, R. (1996c) Nothofagirhiza vinicolor. In Colour Atlas of Ectomycorrhizae (R. Agerer ed.). pl. 98. Einhorn Verlag, Schwäbisch Gmünd.

Palfner, G. \& Godoy, R. (1996d) Russula fuegiana. In Colour Atlas of Ectomycorrhizae (R. Agerer, ed.): pl. 106. Einhorn Verlag, Schwäbisch Gmünd.

Peter, M., Ayer, F., Egli, S. \& Honegger, R. (2001) Above and belowground community structure of ectomycorrhizal fungi in three Norway spruce Picea abies stands in Switzerland. Canadian Journal of Botany 79: 1134-1151.

Pigott, C. D. (1982) Fine structure of mycorrhiza formed by Cenococcum geophilum Fr. on Tilia cordata Mill. New Phytologist 92: $501-512$.

Pillukat, A. (1996) Lactarius salmonicolor R. Heim \& Leclair + Abies alba Mill. Descriptions of Ectomycorrhizae 1: 59-64.

Pillukat, A. (1998) Lactarius salmonicolor. In Colour Atlas of Ectomycorrhizae (R. Agerer, ed.): pl. 122. Einhorn Verlag, Schwäbisch Gmünd.

Pirazzi, R. (1998) Morfologia delle micorrize di tartufi minori: Tuber rufum Pico, T. panniferum Tull. e T. excavatum Vitt. Micologia Italiana 1998 (1): 77-83.

Pritsch, K., Munch, J. C. \& Buscot, F. (1997) Morphological and anatomical characterization of black alder Alnus glutinosa (L.) Gaertn. ectomycorrhizas. Mycorrhiza 7: 201-216.

Raidl, S. (1998a) Rhizopogon melanogastroides. In Colour Atlas of Ectomycorrhizae (R. Agerer, ed.): pl. 135. Einhorn Verlag, Schwäbisch Gmünd.

Raidl, S. (1998b) Tomentella ferruginea. In Colour Atlas of Ectomycorrhizae (R. Agerer, ed.): pl. 137. Einhorn Verlag, Schwäbisch Gmünd.

Raidl, S. (1999) Chamonixia caespitosa Rolland + Picea abies (L) Karst. Descriptions of Ectomycorrhizae 1: 23-28.

Raidl, S. \& Agerer, R. (1998a) Hysterangium stoloniferum Tul \& Tul. + Picea abies (L) Karst. Descriptions of Ectomycorrhizae 3: 31-35.

Raidl, S. \& Agerer, R. (1998b) Rhizopogon roseolus (Corda) Th. M. Fr. + Pinus sylvestris L. Descriptions of Ectomycorrhizae 3: $105-110$.

Raidl, S. \& Müller, W. R. (1996) Tomentella ferruginea (Pers.) Pat. +Fagus sylvatica L. Descriptions of Ectomycorrhizae 1: 161-166.

Raidl, S., Beenken, L. \& Agerer, R. (1998) Rhizopogon melanogastroides M. Lange+Pinus mugo Turra. Descriptions of Ectomycorrhizae 3: 99-104.

Rauscher, T. \& Chevalier, G. (1995a) Tuber melanosporum. In Colour Atlas of Ectomycorrhizae (R. Agerer, ed.): pl. 87. Einhorn Verlag, Schwäbisch Gmünd.

Rauscher, T. \& Chevalier, G. (1995b) Tuber mesentericum. In Colour Atlas of Ectomycorrhizae (R. Agerer, ed.): pl. 88. Einhorn Verlag, Schwäbisch Gmünd.

Rauscher, T. \& Chevalier, G. (1995c) Tuber rufum. In Colour Atlas of Ectomycorrhizae (R. Agerer, ed.): pl. 89. Einhorn Verlag, Schwäbisch Gmünd. 
Rauscher, T., Agerer, R. \& Chevalier, G. (1995) Ektomykorrhizen von Tuber melanosporum, Tuber mesentericum und Tuber rufum (Tuberales) an Corylus avellana. Nova Hedwigia 61 (3-4): 281-322.

Rauscher, T., Müller, W. R., Agerer, R. \& Chevalier, G. (1996a) Tuber borchii Vitt. + Corylus avellana L. Descriptions of Ectomycorrhizae 1: 173-178.

Rauscher, T., Müller, W. R., Chevalier, G. \& Agerer, R. (1996b) Tuber aestivum. In Colour Atlas of Ectomycorrhizae (R. Agerer, ed.): pl. 112. Einhorn Verlag, Schwäbisch Gmünd.

Rauscher, T., Müller, W. R., Chevalier, G. \& Agerer, R. (1996c) Tuber borchii. In Colour Atlas of Ectomycorrhizae (R. Agerer, ed.): pl. 113. Einhorn Verlag, Schwäbisch Gmünd.

Richter, D. L. \& Bruhn, J. N. (1990) Scleroderma citrinum (Gasteromycetes, Sclerodermatales) and Larix decidua form ectomycorrhizae in pure culture. Nova Hedwigia 50: 355-360.

Rose, R. W., Gerald Van Dyke, C. \& Davey, C. B. (1981) Scanning electron microscopy of three types of ectomycorrhizae formed on Eucalyptus nova-anglica in the southeastern United States. Canadian Journal of Botany 59: 683-688.

Rubini, A., Paolocci, F., Granetti, B. \& Arcioni, S. (2001) Morphological characterization of molecular-typed Tuber magnatum ectomycorrhizae. Mycorrhiza 11: 179-185.

Sáez, R. \& De Miguel, A. M. (1995) La trufa negra: Tuber melanosporum Vitt. Guia práctica de truficultura. ITGA, Universidad de Navarra, Pamplona.

Scannerini, S. \& Palenzona, M. (1967) Ricerche sulle ectomicorrize di Pinus strobus in vivaio. III. Micorrize di Tuber albidum Pico. Allionia 13: 187-194.

Schramm, J. R. (1966) Plant colonization studies on black wastes from anthracite mining in Pennsylvania. Transactions of the American Philosophical Society 56: 1-194.

See, L. S., Alexander, I. J. \& Watling, R. (1997) Ectomycorrhizas and putative ectomycorrhizal fungi of Shorea leprosula Miq. (Dipterocarpaceae). Mycorrhiza 7: 63-81.

Selosse, M.-A., Bauer, R. \& Moyersoen, B. (2002) Basal hymenomycetes belonging to the Sebacinaceae are ectomycorrhizal on temperate deciduous forests. New Phytologist 155: 183-195.

Stendell, E. R., Horton, T. R. \& Bruns, T. D. (1999) Early effects of prescribed fire on the structure of the ectomycorrhizal fungus community in a Sierra Nevada ponderosa pine forest. Mycological Research 103: 1353-1359.

Taylor, A. F. S. \& Alexander, I. J. (1991) Ectomycorrhizal synthesis with Tylospora fibrillosa, a member of the Corticiaceae. Mycological Research 95: 381-384.

Taylor, D. L. \& Bruns, T. D. (1999) Community structure of ectomycorrhizal fungi in a Pinus muricata forest: minimal overlap between the mature forest and resistant propagule communities. Molecular Ecology 8: 1837-1850.

Tedersoo, L., Kõljalg, U., Hallenberg, N. \& Larsson, K.-H. (2003) Fine scale distribution of ectomycorrhizal fungi and roots across substrate layers including coarse woody debris in a mixed forest. New Phytologist 159: 153-165.

Thomas, G. W. \& Jackson, R. M. (1979) Sheathing mycorrhizas of nursery grown Picea sitchensis. Transactions of the British Mycological Society 73 (1): 117-125.

Torres, P. \& Honrubia, M. (1994) Ectomycorrhizal associations proven for Pinus halepensis. Israel Journal of Plant Sciences 42: $51-58$.

Torres, P. \& Honrubia, M. (1997) Changes and effects of a natural fire on ectomycorrhizal inoculum potential of soil in a Pinus halepensis forest. Forest Ecology and Management 96: 189-196.

Torres, P., Roldán, A., Lansac, A. R. \& Martín, A. (1995) Ectomycorrhizal formation between Cistus ladanifer and Laccaria laccata. Nova Hedwigia 60: 311-315.

Trappe, M., Eberhart, J. \& Luoma, D. M. (2000) Craterellus tubaeformis (Fr.) Quélet. + Tsuga heterophylla (Raf.) Sarg. In A Manual of Concise Descriptions of North American Ectomycorrhizae: including microscopic and molecular characterization (D. M. Goodman, D. M. Durall, J. A. Trofymow \& S. Berch, eds): CDE 23. Mycologue Publications, Sidney, BC.
Treu, R. (1990a) Charakterisierung und Identifizierung von Ektomykorrhizen aus dem Nationalpark Berchtesgaden. Bibliotheca Mycologica 134: 1-196.

Treu, R. (1990b) Boletinus cavipes. In Colour Atlas of Ectomycorrhizae (R. Agerer, ed.): pl. 35. Einhorn Verlag, Schwäbisch Gmünd.

Treu, R. (1990c) Hebeloma edurum. In Colour Atlas of Ectomycorrhizae (R. Agerer, ed.): pl. 39. Einhorn Verlag, Schwäbisch Gmünd.

Treu, R. (1990d) Lactarius alpinus. In Colour Atlas of Ectomycorrhizae (R. Agerer, ed.): pl. 40. Einhorn Verlag, Schwäbisch Gmünd.

Treu, R. (1990e) Lactarius badiosanguineus. In Colour Atlas of Ectomycorrhizae (R. Agerer, ed.): pl. 41. Einhorn Verlag, Schwäbisch Gmünd.

Treu, R. (1990f) Lactarius porninsis. In Colour Atlas of Ectomycorrhizae (R. Agerer, ed.): pl. 42. Einhorn Verlag, Schwäbisch Gmünd.

Treu, R. (1990g) Laricirhiza alpina. In Colour Atlas of Ectomycorrhizae (R. Agerer, ed.): pl. 50. Einhorn Verlag, Schwäbisch Gmünd.

Treu, R. (1990h) Russula firmula. In Colour Atlas of Ectomycorrhizae (R. Agerer, ed.): pl. 43. Einhorn Verlag, Schwäbisch Gmünd.

Treu, R. (1990i) Russula laricina. In Colour Atlas of Ectomycorrhizae (R. Agerer, ed.): pl. 44. Einhorn Verlag, Schwäbisch Gmünd.

Treu, R. (1990j) Suillus flavus. In Colour Atlas of Ectomycorrhizae (R. Agerer, ed.): pl. 45. Einhorn Verlag, Schwäbisch Gmünd.

Treu, R. (1990k) Suillus plorans. In Colour Atlas of Ectomycorrhizae (R. Agerer, ed.): pl. 46. Einhorn Verlag, Schwäbisch Gmünd.

Treu, R. (1990l) Suillus sibiricus. In Colour Atlas of Ectomycorrhizae (R. Agerer, ed.): pl. 47. Einhorn Verlag, Schwäbisch Gmünd.

Treu, R. (1993) Suillus laricinus. In Colour Atlas of Ectomycorrhizae (R. Agerer, ed.): pl. 81. Einhorn Verlag, Schwäbisch Gmünd.

Trost, T., Agerer, R., Urbancic, M. \& Kraigher, H. (1999) Biodiversity of ectomycorrhizae in a Norway spruce stand on Pokljuka. Phyton 39 (4): 225-232.

Uhl, M. (1988a) Studies on ectomycorrhizae XVI. Ectomycorrhizae formed by Tricholoma flavobrunneum and Betula pendula and Tricholoma auratum and Pinus sylvestris. Mycotaxon 33: $1-21$.

Uhl, M. (1988b) Rhizopogon luteolus. In Colour Atlas of Ectomycorrhizae (R. Agerer, ed.): pl. 21. Einhorn Verlag, Schwäbisch Gmünd.

Uhl, M. (1989) Tylopilus felleus. In Colour Atlas of Ectomycorrhizae (R. Agerer, ed.): pl. 33. Einhorn Verlag, Schwäbisch Gmünd.

Uhl, M. \& Agerer, R. (1987) Studies on ectomycorrhizae XI. Mycorrhizae formed by Dermocybe crocea on Pinus sylvestris and Dermocybe palustris on Pinus mugo. Nova Hedwigia 45 (3-4): $509-527$

Uhl, M. \& Agerer, R. (1988) Dermocybe crocea. In Colour Atlas of Ectomycorrhizae (R. Agerer, ed.): pl. 14. Einhorn Verlag, Schwäbisch Gmünd.

Urban, A., Weiss, M. \& Bauer, R. (2003) Ectomycorrhizas involving sebacinoid mycobionts. Mycological Research 107: 3-14.

Ursic, M. \& Peterson, R. L. (1997) Morphological and anatomical characterization of ectomycorrhizas and ectendomycorrhizas on Pinus strobus seedlings in a southern Ontario nursery. Canadian Journal of Botany 75: 2057-2072.

Voiry, H. (1981) Classification morphologique des ectomycorhizes du chêne et du hêtre dans le nord-est de la France. European Journal of Forest Pathology 11: 284-299.

Waller, K. \& Agerer, R. (1993) Scleroderma citrinum. In Colour Atlas of Ectomycorrhizae (R. Agerer, ed.): pl. 80. Einhorn Verlag, Schwäbisch Gmünd.

Waller, K., Brand, F., Agerer, R. \& Taylor, A. (1993) Piceirhiza oleiferans. In Colour Atlas of Ectomycorrhizae (R. Agerer, ed.): pl. 79. Einhorn Verlag, Schwäbisch Gmünd.

Watling, R., Taylor, A., See, L. S., Sims, K. \& Alexander, I. (1995b) A rainforest Pisolithus; its taxonomy and ecology. Nova Hedwigia 61: 417-429. 
Watling, R., Taylor, A. F. S., See, L. S., Sims, K. \& Alexander, I. J. (1995a) Pisolithus aurantioscabrosus. In Colour Atlas of Ectomycorrhizae (R. Agerer, ed.): pl. 85. Einhorn Verlag, Schwäbisch Gmünd.

Weiss, M. (1989) Amphinema byssoides. In Colour Atlas of Ectomycorrhizae (R. Agerer, ed.): pl. 23. Einhorn Verlag, Schwäbisch Gmünd.

Weiss, M. (1991a) Studies on ectomycorrhizae XXXIII. Descriptions of three mycorrhizae synthesized on Picea abies. Mycotaxon 40: 53-77.

Weiss, M. (1991b) Pisolithus tinctorius. In Colour Atlas of Ectomycorrhizae (R. Agerer, ed.): pl. 63. Einhorn Verlag, Schwäbisch Gmünd.

Weiss, M. (1992) Mycorrhizae formed by Pisolithus tinctorius (Basidiomycetes) on Norway spruce. Cryptogamic Botany 2: 337-344.

Wiedmer, E. \& Senn-Irlet, B. (1998) Cortinarius pluvius (Fr.) Fr. + Alnus viridis (Chaix) DC. Descriptions of Ectomycorrhizae 3: $31-36$.

Wiedmer, E. \& Senn-Irlet, B. (1999a) Cortinarius alnobetulae Kühn. + Alnus viridis (Chaix) DC. Descriptions of Ectomycorrhizae 4: 7-12.

Wiedmer, E. \& Senn-Irlet, B. (1999b) Cortinarius atropusillus Favre + Alnus viridis (Chaix) DC. Descriptions of Ectomycorrhizae 4: $13-18$.

Wiedmer, E. \& Senn-Irlet, B. (1999c) Cortinarius badiovestitus Mos. + Alnus viridis (Chaix) DC. Descriptions of Ectomycorrhizae 4: 19-23.

Wiedmer, E. \& Senn-Irlet, B. (1999d) Cortinarius kühneri Mos. + Alnus viridis (Chaix) DC. Descriptions of Ectomycorrhizae 4: 25-29.

Wiedmer, E. \& Senn-Irlet, B. (2001a) Cortinarius bibulus Quél. + Alnus viridis (Chaix) DC. Descriptions of Ectomycorrhizae 5: $23-27$.

Wiedmer, E. \& Senn-Irlet, B. (2001b) Cortinarius helvelloides (Fr.) Fr. + Alnus viridis (Chaix) DC. Descriptions of Ectomycorrhizae 5: 29-34.

Wiedmer, E. \& Senn-Irlet, B. (2004) Lactarius lepidotus Smith \& Hesler + Alnus viridis (Chaix) DC. Descriptions of Ectomycorrhizae 7-8: 45-48.

Wiedmer, E., Senn-Irlet, B. \& Agerer, R. (2001) Alpova diplophloeus (Zeller \& Dodge) Trappe \& A. H. Smith + Alnus viridis (Chaix) DC. Descriptions of Ectomycorrhizae 5: 1-8.

Wiedmer, E., Senn-Irlet, B., Hahn, Ch. \& Agerer, R. (2004) Melanogaster broomeianus Berk. Ex Tul.+Alnus viridis (Chaix) DC. Descriptions of Ectomycorrhizae 7-8: 49-57.

Wöllecke, J. (2001) Charakterisierung der Mykorrhizazönosen zweier Kiefernforste unterschiedlicher Trophie. [Cottbuser Schriften zu Bodenschutz und Rekultivierung Vol. 17.] Brandenburgische Technische Universität Cottbus. Druckzone, Cottbus.

Wöllecke, J., Münzenberger, B. \& Hüttl, R. (1997a) 'Pinirhiza arachnorosea' + Pinus sylvestris L. Descriptions of Ectomycorrhizae 2: $37-42$.

Wöllecke, J., Münzenberger, B. \& Hüttl, R. (1997b) 'Pinirhiza luteoalba' + Pinus sylvestris L. Descriptions of Ectomycorrhizae 2: $55-60$.

Wöllecke, J., Münzenberger, B. \& Hüttl, R. (1998a) 'Pinirhiza rufomaculata' + Pinus sylvestris L. Descriptions of Ectomycorrhizae 3: 73-78.

Wöllecke, J., Münzenberger, B. \& Hüttl, R. (1998b) 'Pinirhiza sulphurea' + Pinus sylvestris L. Descriptions of Ectomycorrhizae 3: 79-84.
Wöllecke, J., Münzenberger, B. \& Hüttl, R. (1999a) 'Pinirhiza cephalocystidia' + Pinus sylvestris L. Descriptions of Ectomycorrhizae 4: 67-72.

Wöllecke, J., Münzenberger, B. \& Hüttl, R. (1999b) 'Pinirhiza hyphocystidia' + Pinus sylvestris L. Descriptions of Ectomycorrhizae 4: $85-90$.

Wöllecke, J., Münzenberger, B. \& Hüttl, R. F. (1998c) Pinirhiza arachnorosea. In Colour Atlas of Ectomycorrhizae (R. Agerer, ed.): pl. 125. Einhorn Verlag, Schwäbisch Gmünd.

Wöllecke, J., Münzenberger, B. \& Hüttl, R. F. (1998d) Pinirhiza rufomaculata. In Colour Atlas of Ectomycorrhizae (R. Agerer, ed.): pl. 129. Einhorn Verlag, Schwäbisch Gmünd.

Yamada, A. (1998a) Russula delica Fr.+Betula platyphylla Sukatchev var. japonica Hara. In A Manual of Concise Descriptions of North American Ectomycorrhizae: including microscopic and molecular characterization (D. M. Goodman, D. M. Durall, J. A. Trofymow \& S. Berch, eds): CDE 16. Mycologue Publications, Sidney, BC.

Yamada, A. (1998b) Russula nigricans (Bull.: Fr.) Fr. + Betula platyphylla Sukatchev var. japonica Hara. In A Manual of Concise Descriptions of North American Ectomycorrhizae: including microscopic and molecular characterization (D. M. Goodman, D. M. Durall, J. A. Trofymow \& S. Berch, eds): CDE 17. Mycologue Publications, Sidney, BC.

Yamada, A., Ogura, T. \& Ohmasa, M. (2001) Cultivation of mushrooms of edible ectomycorrhizal fungi associated with Pinus densiflora by in vitro mycorrhizal synthesis. II. Morphology of mycorrhizas in open-pot soil. Mycorrhiza 11: 67-81.

Zak, B. (1971) Characterization and classification of mycorrhizae of Douglas-fir. II. Pseudotsuga menziesii + Rhizopogon vinicolor. Canadian Journal of Botany 49: 1079-1084.

Zak, B. \& Larsen, M. J. (1978) Characterization and classification of mycorrhizae of Douglas-fir. III. Pseudotsuga menziesii+ Byssoporia (Porya) terrestris vars. lilacinorosea, parksii, and sublutea. Canadian Journal of Botany 56: 1416-1424.

Zak, J.C. (1973) Classification of Ectomycorrhizae. In Ectomycorrhizae, their Ecology and Physiology (G. C. Marks \& T. T. Kozlowski, eds): 43-78. Academic Press, New York.

Zambonelli, A., Iotti, M. \& Govi, G. (1998) Caratterizzazione anatomo-morfologica delle micorrize in una tartufaia coltivata di Tuber borchii Vittad. Micologia Italiana 1998 (1): 23-36.

Zambonelli, A., Iotti, M., Amicucci, A. \& Pisi, A. (1999) Caratterizzazione anatomo-morfologica delle micorrize di Tuber maculatum Vittad. su Ostrya carpinifolia. Micologia Italiana 1999 (3): 29-35.

Zambonelli, A., Salomoni, S. \& Pisi, A. (1993) Caratterizzazione anatomo-morfologica delle micorrize di Tuber spp. su Quercus pubescens Willd. Micologia Italiana 1993 (3): 73-90.

Zambonelli, A., Salomoni, S. \& Pisi, A. (1995) Caratterizzazione anatomo-morfologica delle micorrize di Tuber borchii, Tuber aestivum, Tuber mesentericum, Tuber brumale, Tuber melanosporum su Pinus pinea. Micologia Italiana 1995 (2): 119-137.

Zambonelli, A., Tibiletti, E. \& Pisi, A. (1997) Caratterizzazione anatomo-morfologica delle micorrize di Tuber indicum Cooke \& Massee su Pinus pinea L. e Quercus cerris L. Micologia Italiana 1997 (1): 29-36.

Corresponding Editor: J. W. G. Cairney 